Pacific

Journal of

Mathematics

THE CHABAUTY SPACE OF CLOSED SUBGROUPS OF THE THREE-DIMENSIONAL HEISENBERG GROUP

Martin R. Bridson, PierRe de la Harpe And Victor KLEPTSyN

Volume $240 \quad$ No. 1

March 2009 


\title{
THE CHABAUTY SPACE OF CLOSED SUBGROUPS OF THE THREE-DIMENSIONAL HEISENBERG GROUP
}

\author{
Martin R. BRidson, PierRe De la Harpe And Victor KLEPTSyn
}

\begin{abstract}
When equipped with the natural topology first defined by Chabauty, the closed subgroups of a locally compact group $G$ form a compact space $\mathscr{C}(G)$. We analyse the structure of $\mathscr{C}(G)$ for some low-dimensional Lie groups, concentrating mostly on the 3-dimensional Heisenberg group $H$. We prove that $\mathscr{C}(H)$ is a 6-dimensional space that is path-connected but not locally connected. The lattices in $H$ form a dense open subset $\mathscr{L}(H) \subset \mathscr{C}(H)$ that is the disjoint union of an infinite sequence of pairwise homeomorphic aspherical manifolds of dimension six, each a torus bundle over $\left(S^{3} \backslash T\right) \times R$, where $T$ denotes a trefoil knot. The complement of $\mathscr{L}(H)$ in $\mathscr{C}(H)$ is also described explicitly. The subspace of $\mathscr{C}(H)$ consisting of subgroups that contain the centre $Z(H)$ is homeomorphic to the 4 -sphere, and we prove that this is a weak retract of $\mathscr{C}(H)$.
\end{abstract}

\section{Introduction}

Let $G$ be a locally compact topological group. We denote by $\mathscr{C}(G)$ the space of closed subgroups of $G$ equipped with the Chabauty topology; this is a compact space. A basis of neighbourhoods for a closed subgroup $C \in \mathscr{C}(G)$ is formed by the subsets

$$
\mathscr{V}_{K, U}(C)=\{D \in \mathscr{C}(G) \mid D \cap K \subset C U \text { and } C \cap K \subset D U\},
$$

where $K \subseteq G$ is compact and $U$ is an open neighbourhood of the identity $e \in G$. We will consider the induced topology on various subspaces of $\mathscr{C}(G)$, including the space of lattices $\mathscr{L}(G)$, the larger space of discrete subgroups $\mathscr{D}(G)$, the space of abelian closed subgroups $\mathscr{A}(G)$, and the space of normal closed subgroups $\mathcal{N}(G)$.

MSC2000: 22D05, 22E25, 22E40.

Keywords: Chabauty topology, Heisenberg group, space of closed subgroups, space of lattices, affine group.

This work was funded by the Swiss National Science Foundation. Bridson was also supported in part by a Senior Fellowship from the EPSRC (UK) and a Royal Society Wolfson Research Merit Award, while Kleptsyn was supported in part by the Russian scholarship RFBR 07-01-00017-a and in part by the French-Russian scholarship RFBR CNRS-L-a 05-01-02801. 
$\mathscr{C}(G)$ is contained in the set of all closed subspaces of the topological space $G$. There is a natural topology on this larger set, the study of which goes back in some form to Hausdorff and Vietoris; we recall some of its basic properties in Section 3. The Chabauty topology coincides with the restriction of this topology to $\mathscr{C}(G)$.

Chabauty's purpose in introducing this topology was to generalize a criterion of Mahler that allows one to prove that appropriate sets of lattices are relatively compact. Mahler's original work concerned lattices in $\mathbf{R}^{n}$, while Chabauty's generalization [1950] deals with lattices in a large class of locally compact groups; this result is known as the Mahler-Chabauty compactness criterion. Two early references concerning the Chabauty topology are [Macbeath and Świerczkowski 1960] and [Bourbaki 1963, chapitre 8, § 5]. The Chabauty topology is also induced by a topology defined by Fell on spaces of closed subsets [Fell 1962].

The topology has been useful for the study of Fuchsian groups [Harvey 1977, in particular, Section 2] and more generally of discrete subgroups of other semisimple groups [Guivarc'h and Rémy 2006], as well as hyperbolic manifolds; see [Thurston 1979, Chapter 9] and [Canary et al. 1987, in particular, Section I.3.1]; for Fuchsian and Kleinian groups, convergence with respect to the Chabauty topology is known as geometric convergence. Observe that whenever a space $X$ can be identified with a family of closed subgroups of a locally compact group $G$, as is the case for Riemannian symmetric spaces or Bruhat-Tits buildings, the closure of $X$ in $\mathscr{C}(G)$ provides a natural compactification of $X$, which is in many cases an efficient tool for studying $X$ (this is explicit in, for example, [Guivarc'h et al. 1998, Chapter IX], [Guivarc'h and Rémy 2006], and [Borel and Ji 2006, Sections I.17 and III.17-19]). We also draw the reader's attention to [Ghys 2007] where, by studying a certain flow on the space $\mathscr{L}^{\text {umod }}(\mathbf{C})$ of coarea-1 lattices in $\mathbf{C}$, Ghys discovered fascinating phenomena linking dynamics, knot theory and arithmetic.

When the group $G$ is discrete, the Chabauty topology on $\mathscr{C}(G)$ coincides with the restriction to $\mathscr{C}(G)$ of the product topology on the compact space $2^{G}$ of all subsets of $G$. In particular, if $F_{k}$ is the free group on $k$ generators, $\mathcal{N}\left(F_{k}\right)$ is the space of marked groups on $k$ generators, which has been the subject of much work since its appearance in the final remarks of [Gromov 1981]; see among others [Grigorchuk 1984; Champetier 2000; Champetier and Guirardel 2005; de Cornulier et al. 2007; 2008].

Our purpose in this work is to describe some examples of spaces $\mathscr{C}(G)$. A theme that the reader will observe quickly is that for the most easily understood of lowdimensional Lie groups the space $\mathscr{C}(G)$ can be complicated and yet beguilingly tractable. 
As a preliminary exercise, we consider in Section 2 the group

$$
\operatorname{Aff}=\left\{\left(\begin{array}{cc}
e^{s} & t \\
0 & 1
\end{array}\right) \mid s, t \in \mathbf{R}\right\}
$$

of orientation preserving affine transformations of the real line. Up to isomorphism, this is the unique nonabelian connected real Lie group of dimension 2.

Proposition 1.1. The space $\mathscr{C}(\mathrm{Aff})$ is a compact, contractible space of dimension 2. More precisely, it is the union of a closed 2-disc $\mathbf{D}$ and a compact interval I attached by one of its end points to a point on the boundary circle $\partial \mathbf{D}$.

The disc $\mathbf{D}$ arises as a cone over the real projective line formed by the subgroups isomorphic to $\mathbf{R}$; the cone point is the trivial subgroup $\{e\}$.

The subspace $\mathcal{N}$ (Aff) consisting of the closed normal subgroups of Aff is the disjoint union of the point $\{e\}$ and the closed interval $I$; in particular, $\mathcal{N}$ (Aff) is disconnected.

In Section 3, we recall some general facts about the Chabauty topology. In Section 4, we revisit the following result of [Hubbard and Pourezza 1979], proving a more detailed version than stated here (Theorem 4.6 versus Theorem 1.2). This result is needed in our analysis of the 3-dimensional Heisenberg group, which is our main interest.

Some notation. For locally compact groups $G, S, \ldots, T$, we denote by $\mathscr{C}_{S, \ldots, T}(G)$ the subspace of $\mathscr{C}(G)$ of closed subgroups isomorphic to one of $S, \ldots, T$. We write $\Sigma X$ to denote the suspension of a space $X$.

We write $\approx$ to indicate homeomorphism of topological spaces and $\cong$ to indicate isomorphism of groups.

Theorem 1.2 (Hubbard and Pourezza). The space $\mathscr{C}(\mathbf{C})$ is homeomorphic to the four-dimensional sphere $\mathbf{S}^{4}$.

More precisely, there is a homeomorphism $\mathscr{C}(\mathbf{C}) \rightarrow \mathbf{S}^{4}=\Sigma \mathbf{S}^{3}$ with the following properties: It identifies $\mathscr{C}_{\mathbf{R}}(\mathbf{C})$ with a trefoil knot $T$ in the equator $\mathbf{S}^{3} \subset \mathbf{S}^{4}$; it maps $\{e\}$ and $\mathbf{C}$ to the south and north poles $\mathbf{s}, \mathbf{n} \in \mathbf{S}^{4}$, respectively; it sends the space of lattices $\mathscr{L}(\mathbf{C})$ onto the complement of the (not locally flat) 2-sphere $\Sigma T \subset \Sigma \mathbf{S}^{3}$; and it identifies $\mathscr{C}_{\mathbf{Z}}(\mathbf{C})$ and $\mathscr{C}_{\mathbf{R} \oplus \mathbf{Z}}(\mathbf{C})$ with the connected components of $\Sigma T \backslash(T \cup\{\mathbf{s}, \mathbf{n}\})$.

We want to emphasize how remarkable it is that $\mathscr{C}\left(\mathbf{R}^{2}\right)$ is a manifold: Even when one knows the homeomorphism type of the subspaces corresponding to the different types of subgroups, it is by no means obvious that they assemble to form a sphere. For $n \geq 3$, the space $\mathscr{C}\left(\mathbf{R}^{n}\right)$ is much wilder: The space of lattices $\mathscr{L}\left(\mathbf{R}^{n}\right) \approx$ $\mathrm{GL}_{n}(\mathbf{R}) / \mathrm{GL}_{n}(\mathbf{Z})$ continues to provide a dense connected open set of dimension $n^{2}$ 
and the lower-dimensional subspaces $\mathscr{C}_{\mathbf{R}^{a} \oplus \mathbf{Z}^{b}}\left(\mathbf{R}^{n}\right)$ are easy to describe, but the manner in which they assemble is not.

Another space that can be described in some detail is $\mathscr{C}(\mathrm{SO}(3))$. This space is neither connected nor locally connected (like $\mathscr{C}(\mathbf{Z})$ ). In more detail, the space of torus subgroups is the base space of the Hopf-like fibration $\mathrm{SO}(3) \rightarrow \mathbf{P}^{2}$, and if $T$ is such a torus subgroup, then any neighbourhood of $T$ in $\mathscr{C}(\mathrm{SO}(3))$ contains finite cyclic groups of arbitrarily large orders.

Beginning in Section 5, we will concentrate on the 3-dimensional Heisenberg group $H$. For the most part we shall work with the model of this group in which

as a set, $H=\mathbf{C} \times \mathbf{R}$,

the product is given by $(z, t)\left(z^{\prime}, t^{\prime}\right)=\left(z+z^{\prime}, t+t^{\prime}+\frac{1}{2} \operatorname{Im}\left(\bar{z} z^{\prime}\right)\right)$,

and there is a homomorphism $p:(z, t) \mapsto z$ from $H$ onto $\mathbf{C}$.

This model (1-3) for the Heisenberg group is isomorphic to the standard matrix model via the isomorphism

$$
\mathbf{C} \times \mathbf{R} \ni(x+i y, t) \mapsto \exp \left(\begin{array}{lll}
0 & x & t \\
0 & 0 & y \\
0 & 0 & 0
\end{array}\right)=\left(\begin{array}{ccc}
1 & x & t+\frac{1}{2} x y \\
0 & 1 & y \\
0 & 0 & 1
\end{array}\right) \in\left(\begin{array}{ccc}
1 & \mathbf{R} & \mathbf{R} \\
0 & 1 & \mathbf{R} \\
0 & 0 & 1
\end{array}\right) .
$$

$H$ is also isomorphic to the semidirect product $\mathbf{R} \ltimes_{u} \mathbf{R}^{2}$ associated to the unipotent action $u: \mathbf{R} \times \mathbf{R}^{2} \rightarrow \mathbf{R}^{2}$ defined by

$$
\left(x,\left(\begin{array}{l}
t \\
y
\end{array}\right)\right) \mapsto\left(\begin{array}{ll}
1 & x \\
0 & 1
\end{array}\right)\left(\begin{array}{l}
t \\
y
\end{array}\right)=\left(\begin{array}{c}
t+x y \\
y
\end{array}\right) .
$$

Our examination of the structure of $\mathscr{C}(H)$ spans Sections 5 to 8 . We summarize the main results of these sections in the following two theorems. The first provides an overview of the global structure of $\mathscr{b}(H)$, and the second gives more information about the strata corresponding to the various types of subgroups as well as the manner in which $\operatorname{Aut}(H)$ acts on them.

We define subspaces $\mathscr{L}_{n}(H) \subset \mathscr{L}(H)$ by declaring that a lattice $\Lambda$ is in $\mathscr{L}_{n}(H)$ if $[\Lambda, \Lambda]$ has index $n$ in $\Lambda \cap Z(H)$. The subset $\mathscr{L}_{\infty}(H) \subset \mathscr{C}(H)$ is defined to consist of the subgroups $p^{-1}(L)$ with $L \in \mathscr{L}(\mathbf{C})$. And $\mathscr{L}_{! !}(H)=\mathscr{L}(H) \cup \mathscr{L}_{\infty}(H)$. We define $\mathscr{C}_{\geq Z}(H)$ as the space of closed subgroups of $H$ that contain the centre $Z(H)$; observe that $\mathscr{L}_{\infty}(H) \subset \mathscr{b}_{\geq Z}(H)$.

We write $\mathbf{P}^{1}$ to denote the real projective line and $\mathbf{P}^{2}$ for the real projective plane.

The real projective line $\mathbf{P}^{1}$ is of course homeomorphic to the circle $\mathbf{S}^{1}$. However, in this work, we try to mark the difference between homeomorphism with a circle related to the choice of a unit vector in a plane and homeomorphism with 
a circle related to the choice of a line in a plane; see for example the covering $\mathbf{S}^{1} \approx \widehat{\mathscr{C}}_{\mathbf{R}^{2}}(H) \rightarrow \mathscr{C}_{\mathbf{R}^{2}}(H) \approx \mathbf{P}^{1}$ of (6-5) below.

Theorem 1.3. The compact space $\mathscr{b}(H)$ is arc connected but not locally connected. It can be expressed as the union of the following three subspaces.

(i) $\mathscr{L}(H)$, which is open and dense in $\mathscr{C}(H)$; this has countably many connected components $\mathscr{L}_{n}(H)$, each of which is homeomorphic to a fixed aspherical 6-manifold that is a 2-torus bundle over $\mathscr{L}(\mathbf{C}) \approx \mathrm{GL}_{2}(\mathbf{R}) / \mathrm{GL}_{2}(\mathbf{Z})$.

(ii) $\mathscr{A}(H)$, which is homeomorphic to the space obtained from $\mathbf{S}^{4} \times \mathbf{P}^{1}$ by fixing a tame arc $I \subset \mathbf{S}^{4}$ and collapsing each of the circles $\left\{\{i\} \times \mathbf{P}^{1}: i \in I\right\}$ to a point.

(iii) $\mathscr{C}_{\geq Z}(H)$, from which there is a natural homeomorphism to $\mathbf{S}^{4}$; the complement of $\mathscr{L}_{\infty}(H)$ in $\mathscr{C}_{\geq Z}(H)$ is a 2-sphere $\Sigma^{2} \subset \mathbf{S}^{4}$ (which fails to be locally flat at two points).

The union $\mathscr{A}(H) \cup \mathscr{C}_{\geq Z}(H)$ is the complement of $\mathscr{L}(H)$ in $\mathscr{C}(H)$, the intersection

$$
\mathscr{A}(H) \cap \mathscr{C}_{\geq Z}(H)=\left\{C \in \mathscr{C}_{\geq Z}(H) \mid p(C) \subset \mathscr{C}_{\{0\}, \mathbf{Z}, \mathbf{R}}(\mathbf{C})\right\}
$$

is a closed 2-disc in $\Sigma^{2}$, and the space $\mathscr{L}_{! !}(H)=\mathscr{L}(H) \cup \mathscr{L}_{\infty}(H)$ is precisely $\{C \in \mathscr{C}(H) \mid p(C) \in \mathscr{L}(\mathbf{C})\}$.

$\mathscr{C}_{\geq Z}(H)$ is a weak retract of $\mathscr{C}(H)$ : there is a continuous map $f: \mathscr{C}(H) \rightarrow \mathbf{S}^{4}$, constant on $\mathscr{A}(H)$, such that $f \circ j \simeq \mathrm{id}_{\mathbf{S}^{4}}$, where $j: \mathbf{S}^{4} \rightarrow \mathscr{C}_{\geq Z}(H)$ is the homeomorphism of (iii), and where $\simeq$ denotes homotopy equivalence. In particular, $\pi_{4}(\mathscr{C}(H))$ surjects onto $\mathbf{Z}$.

The subspace $\mathcal{N}(H)$ of normal closed subgroups of $H$ is the union of $\mathscr{C}_{\geq Z}(H)$ (which is homeomorphic to $\mathscr{C}(\mathbf{C}) \approx \mathbf{S}^{4}$ ) and the interval $\{C \in \mathscr{C}(H) \mid C \subset Z(H)\}$, attached to the sphere $\mathscr{C}_{\geq Z}(H)$ by one of its endpoints.

For our second compendium of results concerning $\mathscr{b}(H)$ we need the following notation. We denote by $\mathbf{K}$ the Klein bottle (that is, the total space of the nontrivial $\mathbf{P}^{1}$ bundle over $\left.\mathbf{P}^{1}\right)$. We write $p_{*}^{-1}\left(\mathscr{C}_{\mathbf{R} \oplus \mathbf{Z}}(\mathbf{C})\right)$ for the subspace of $\mathscr{C}(H)$ consisting of closed subgroups $C$ of $H$ with $p(C)$ a subgroup of $\mathbf{C}$ isomorphic to $\mathbf{R} \oplus \mathbf{Z}$; observe that $p_{*}^{-1}\left(\mathscr{C}_{\mathbf{R} \oplus \mathbf{Z}}(\mathbf{C})\right) \subset \mathscr{C}_{\geq Z}(H)$.

Theorem 1.4. The spaces $\mathscr{L}_{n}(H)$ are homeomorphic to a common aspherical homogeneous space, namely the quotient of the 6-dimensional automorphism group $\operatorname{Aut}(H) \cong \mathbf{R}^{2} \rtimes \mathrm{GL}_{2}(\mathbf{R})$ by the discrete subgroup $\mathbf{Z}^{2} \rtimes \mathrm{GL}_{2}(\mathbf{Z})$.

The frontier of $\mathscr{L}_{n}(H)$, which is independent of $n$, consists of the following subspaces:

(i) the trivial group $\{e\}$;

(ii) $\mathscr{C}_{\mathbf{R}}(H) \approx \mathbf{P}^{2}$;

(iii) $\left.\mathscr{C}_{\mathbf{Z}}(H) \approx \mathbf{P}^{2} \times\right] 0, \infty[$; 
(iv) $\mathscr{C}_{\mathbf{R}^{2}}(H) \approx \mathbf{P}^{1}$;

(v) $\left.\mathscr{C}_{\mathbf{R} \oplus \mathbf{Z}}(H) \approx \mathbf{K} \times\right] 0, \infty\left[\right.$, which is a $\left(\mathbf{P}^{1} \times\right] 0, \infty[)$-bundle over $\mathbf{P}^{1}$;

(vi) $\mathscr{C}_{\mathbf{Z}^{2}}(H)$, which is a $\left(\mathbf{S}^{4} \backslash \Sigma^{2}\right)$-bundle over $\mathbf{P}^{1}$;

(vii) $p_{*}^{-1}\left(\mathscr{C}_{\mathbf{R} \oplus \mathbf{Z}}(\mathbf{C})\right)$;

(viii) the full group $H$.

In particular, the frontier of $\mathscr{L}_{n}(H)$, which is independent of $n$, is the union of $\mathscr{A}(H)$ and the complement $\Sigma^{2}$ of $\mathscr{L}_{\infty}(H)$ in $\mathscr{b}_{\geq Z}(H)$; the part $\mathscr{A}(H)$ is itself the union of the subspaces (i) to (vi), and $\Sigma^{2} \backslash\left(\Sigma^{2} \cap \mathscr{A}(H)\right)$ is itself the union of the subspaces (vii) and (viii). The frontier of $\bigcup_{n=1}^{\infty} \mathscr{L}_{n}(H)$ further contains

(ix) $\mathscr{L}_{\infty}(H)$.

Each of these spaces, except (vi), consists of finitely many $\operatorname{Aut}(H)$-orbits.

The action of $\operatorname{Aut}(H)$ on $\mathscr{C}_{\mathbf{Z}^{2}}(H)$, which has uncountably many orbits and which is minimal, is described in the proof of Proposition 6.1 in terms of the standard action of $\mathrm{SL}_{2}(\mathbf{Z})$ on $\mathbf{P}^{1}$.

Observe that, as $\mathscr{L}(H)$ is open dense, the spaces (i) to (ix) of Theorem 1.4 together with the spaces $\mathscr{L}_{n}(H)$ for $n \geq 1$ constitute a partition of $\mathscr{C}(H)$.

The subspaces of $\mathscr{C}(H)$ are described more precisely in later sections. Section 6 describes $\mathscr{A}(H)$ and $\mathscr{C}_{\geq Z}(H)$, Section 7 describes $\mathscr{L}_{n}(H)$, and Section 8 describes $\mathscr{L}_{! !}(H)$ and $\mathscr{C}(H) \backslash \mathscr{A}(H)$.

\section{First examples, including the affine group Aff}

The space $\mathscr{C}(G)$ has a straightforward description when $G$ is a 1-dimensional Lie group: $\mathscr{C}(\mathbf{R})$ is homeomorphic to the closed interval $[0, \infty]$, with $\lambda \in] 0, \infty[$ corresponding to the subgroup $\mathbf{Z} \lambda^{-1}$, and $\mathscr{C}(\mathbf{R} / \mathbf{Z})$ is homeomorphic to $\mathscr{C}(\mathbf{Z})$, which is homeomorphic to $\left\{1, \frac{1}{2}, \frac{1}{3}, \ldots, 0\right\} \subset[0,1]$, with $\frac{1}{n}$ corresponding to the subgroup of index $n$ and 0 corresponding to $\{0\}=\bigcap_{n=1}^{\infty} \mathbf{Z} n$. (In passing, we note that $\mathscr{C}(\mathbf{Z})$ is also homeomorphic to $\mathscr{C}\left(\mathbf{Z}_{p}\right)$ for an arbitrary prime $p$, where $\mathbf{Z}_{p}$ denotes the additive group of $p$-adic integers.)

The direct observation $\mathscr{C}(\mathbf{R} / \mathbf{Z}) \approx \mathscr{C}(\mathbf{Z})$ illustrates the more general fact that if $G$ is a locally compact abelian group with Pontryagin dual $\widehat{G}$, then there is a homeomorphism $\mathscr{C}(G) \rightarrow \mathscr{C}(\widehat{G})$ associating to each closed subgroup $C \subset G$ its orthogonal $C^{\perp}=\{\chi \in \widehat{G} \mid \chi(c)=1$ for all $c \in C\}$. In the case $G=\mathbf{R}$, if we choose an isomorphism of $\widehat{\mathbf{R}}$ with $\mathbf{R}$, the above homeomorphism defines an involution of the interval $[0, \infty]$ that exchanges the two endpoints. 


\section{Closed subgroups of the affine group Aff}

We now turn to an example that is only marginally more involved, namely the affine group of the real line, Aff as described in (1-2). The commutator subgroup [Aff, Aff] is the translation subgroup, described by the equation $s=0$. The following properties are straightforward to check.

- The Lie algebra aff of Aff consists of the set of matrices $\left(\begin{array}{ll}x & y \\ 0 & 0\end{array}\right)$ with $x, y \in \mathbf{R}$.

- The exponential mapping $\mathfrak{a f f} \rightarrow$ Aff is a diffeomorphism given by

$$
\exp \left(\begin{array}{ll}
x & y \\
0 & 0
\end{array}\right)=\left(\begin{array}{cc}
e^{x} & y\left(e^{x}-1\right) / x \\
0 & 1
\end{array}\right)
$$

- Any 1-dimensional subspace of afff distinct from [afff, $\mathfrak{a} \mathfrak{f} f]$ is conjugate under the adjoint representation to the subspace with equation $y=0$; more precisely, when $x_{0} \neq 0$, we have

$$
\left(\begin{array}{cc}
1 & y_{0} / x_{0} \\
0 & 1
\end{array}\right)\left(\begin{array}{cc}
s x_{0} & s y_{0} \\
0 & 0
\end{array}\right)\left(\begin{array}{cc}
1 & -y_{0} / x_{0} \\
0 & 1
\end{array}\right)=\left(\begin{array}{cc}
s x_{0} & 0 \\
0 & 0
\end{array}\right) \quad \text { for any } s \in \mathbf{R} .
$$

- Any $g \in$ Aff with $g \neq e$ lies in a unique one parameter subgroup of Aff which is its centralizer $Z_{\mathrm{Aff}}(g)$.

- Every nonabelian closed subgroup of Aff contains [Aff, Aff].

Consequently, closed subgroups of Aff can be listed as follows:

(i) the trivial group $\{e\}$;

(ii) the infinite cyclic subgroups;

(iii) the one parameter subgroups;

(iv) the groups generated by [Aff, Aff] and one element $g=\left(\begin{array}{cc}\lambda(g) & t \\ 0 & 1\end{array}\right)$ with $\lambda(g)>0$;

(v) the group Aff itself.

For the Chabauty topology, abelian closed subgroups, which are the subgroups of types (i), (ii), and (iii), and which are also the unimodular ${ }^{1}$ closed subgroups, constitute a closed subspace $\mathbf{D}$ of $\mathscr{C}$ (Aff) homeomorphic to the 2-disc; it is natural to regard it as a cone $\left(\mathbf{P}^{1} \times[0, \infty]\right) /((x, 0) \sim(y, 0))$ over the real projective line. The vertex corresponds to the trivial group $\{e\}$, the points in $\mathbf{P}^{1} \times\{\infty\}$ to the one parameter subgroups isomorphic to $\mathbf{R}$, and the other points to the infinite cyclic subgroups (each infinite cyclic subgroup being contained in a unique one parameter subgroup). The parameter $\lambda(g)$ defines a homeomorphism from the subspace formed by subgroups of type (iv) to the open interval $] 0, \infty[$. The closure of this

\footnotetext{
${ }^{1}$ Compare with [Bourbaki 1963, chapitre $8, \S 5$, théorème 1.i]: if $G$ is a locally compact group, the subset of unimodular closed subgroups is closed in $\mathscr{C}(G)$.
} 
subspace is a compact interval with endpoints Aff and [Aff, Aff]. It intersects $\mathbf{D}$ in a single point, namely the point in $\mathbf{P}^{1} \times\{\infty\}$ corresponding to [Aff, Aff].

The proof of Proposition 1.1 is complete.

\section{A reminder concerning the Chabauty topology}

Aspects of Proposition 1.1 and Theorems 1.2 to 1.4 illustrate properties that hold in more general groups. We record some of these in Proposition 3.4 for future reference. Two preliminary lemmas are required.

Lemma 3.1. Let $G$ be a topological group. Let $K$ be a compact subset, and let $V_{1}, \ldots, V_{n}$ be open subsets of $G$ such that $K \subset \bigcup_{j=1}^{n} V_{j}$. Then there exists a neighbourhood $U$ of e such that, for all $x \in K$, there exists $j \in\{1, \ldots, n\}$ with $U x \subset V_{j}$.

Remark. This is strongly reminiscent of (and inspired by) the so-called Lebesgue number lemma, according to which if $\mathscr{V}$ is an open covering of a compact metric space $X$, there exists $\epsilon>0$ such that every subset of $X$ of diameter less than $\epsilon$ is contained in an element $V$ of $\mathscr{V}$. See for example [Munkres 1975, Section 3.7].

Proof. For each $y \in K$, choose $j=j(y)$ such that $y \in V_{j}$. Then $V_{j} y^{-1}$ is a neighbourhood of $e$ and there exist neighbourhoods $U_{y}$ and $U_{y}^{\prime}$ of $e$ such that $U_{y} U_{y}^{\prime} \subset V_{j} y^{-1}$. Set $W_{y}=U_{y}^{\prime} y$; this is a neighbourhood of $y$, and $U_{y} W_{y} \subset V_{j}$.

From the open cover $\left(W_{y}\right)_{y \in K}$ we extract a finite subcover indexed by (say) $y_{1}, \ldots, y_{N} \in K$. Set $U=U_{y_{1}} \cap \cdots \cap U_{y_{N}}$. Then, for any $x \in K \subset \bigcup_{k=1}^{N} W_{y_{k}}$, we have $U x \subset U_{y_{k}} W_{y_{k}} \subset V_{j}$ for some $k \in\{1, \ldots, N\}$ and $j=j\left(y_{k}\right)$.

Recall that a closed subgroup $C$ of $G$ is cocompact if there exists a compact subset $K$ of $G$ such that $G=C K$. A lattice in a locally compact group $G$ is a discrete subgroup $\Lambda$ such that there exists a $G$-invariant finite probability measure on $G / \Lambda$.

For subsets $A, B, \ldots$ and elements $g, h, \ldots$ of a group $G$, we will denote by $\langle A, B, \ldots, g, h, \ldots\rangle$ the subgroup of $G$ generated by $A \cup B \cup \cdots \cup\{g, h, \ldots\}$. (This subgroup need not be closed.)

Lemma 3.2. Let $G$ be a topological group that is compactly generated, let $K$ be a compact generating set of $G$ such that $e \in K$ and $K^{-1}=K$, and let $W$ be a relatively compact nonempty open subset of $G$. Let $c_{1}, \ldots, c_{n} \in G$ be such that

$$
\bar{W} K \subset \bigcup_{j=1}^{n} c_{j} W .
$$

Then there exists a symmetric neighbourhood $U$ of $e$ in $G$ such that, for any $d_{1}, \ldots, d_{n} \in G$ with $d_{j} \in U c_{j}$ for all $j=1, \ldots, n$, we have $\left\langle d_{1}, \ldots, d_{n}\right\rangle W=G$. In particular, the closed subgroup $\overline{\left\langle d_{1}, \ldots, d_{n}\right\rangle}$ is cocompact. 
Proof. Let us first check that condition (3-1) is open in $c_{1}, \ldots, c_{n}$.

By the previous lemma, there exists a neighbourhood $U$ of $e$ in $G$ such that, for any $x \in \bar{W} K$, there exists $j \in\{1, \ldots, n\}$ with $U x \subset c_{j} W$. There is no loss of generality if we also assume that $U^{-1}=U$. For $j \in\{1, \ldots, n\}$, consider any $d_{j} \in U c_{j}$. Let $u_{j} \in U$ be such that $d_{j}=u_{j} c_{j}$. For $x \in \bar{W} K$ with $U x \subset c_{j} W$, we have $u_{j}^{-1} x \in c_{j} W$, namely $x \in d_{j} W$. Hence

$$
\bar{W} K \subset \bigcup_{j=1}^{n} d_{j} W
$$

Set $F=\left\{d_{1}, \ldots, d_{n}\right\}$; now (3-2) reads $F W \supset \bar{W} K$. By induction on $m$, we have

$$
F^{m} W \supset F^{m-1} \bar{W} K \supset F^{m-1} W K \supset \bar{W} K^{m}
$$

for any $m \geq 2$. Consequently $\langle F\rangle W \supset \bar{W}\langle K\rangle$; note that, at this point, we have used the hypothesis $e \in K$ and $K^{-1}=K$. Hence $\langle F\rangle W \supset \bar{W} G=G$, and in particular $\overline{\langle F\rangle}$ is cocompact.

Remark 3.3. Upon replacing $U$ by $U^{\prime}=\bigcap_{k=1}^{n} c_{k}^{-1} U c_{k}$, we can add the following conclusion to Lemma 3.2: For any $d_{1}, \ldots, d_{n} \in G$, if $d_{j} \in c_{j} U^{\prime}$ for $j=1, \ldots, n$, then the closed subgroup $\overline{\left\langle d_{1}, \ldots, d_{n}\right\rangle}$ is cocompact.

For a Hausdorff topological group, the following two properties are clearly equivalent: (i) every neighbourhood of the identity contains a nontrivial subgroup, and (ii) every neighbourhood of the identity contains a nontrivial closed subgroup. (Indeed, for any neighborhood $U$ of the identity, there exists a neighborhood $V$ of the identity such that $\bar{V} \subset U$; for any subgroup $S$ inside $V$, the closure $\bar{S}$ is a closed subgroup inside $U$.) Recall that, by definition, a group has no small subgroup, or is NSS, if these properties do not hold.

Proposition 3.4. Let $G$ be a locally compact group.

(i) The space $\mathscr{A}(G)$ of closed abelian subgroups of $G$ is closed in $\mathscr{C}(G)$.

(ii) The space $\mathscr{K}(G)$ of cocompact closed subgroups of $G$ is open in $\mathscr{C}(G)$ if and only if $G$ is compactly generated.

(iii) If $G$ is NSS, the space $\mathscr{D}(G)$ of discrete subgroups of $G$ is open in $\mathscr{C}(G)$.

(It follows that, if $G$ is compactly generated, the space of cocompact lattices of $G$ is open in $\mathscr{C}(G)$.)

Remarks. Claim (ii) is essentially a result of Stieglitz and Oler [Oler 1973]. Compare (ii) with [Bourbaki 1963, chapitre $8, \S 5, \mathrm{n}^{\mathrm{o}} 3$, théorème $1(\mathrm{i})$, (ii)]: If $\mathcal{U}(G)$ denotes the space of unimodular closed subgroups of $G$, then $U(G)$ is closed in $\mathscr{C}(G)$. If, moreover, $G$ is compactly generated, then $\mathscr{K}(G) \cap U(G)$ is open in $\mathcal{U}(G)$. 
Compare (iii) with [Bourbaki 1963, chapitre $8, \S 5, \mathrm{n}^{\circ} 4$, théorème 2(i), (iii)]: If $G$ is NSS, then $\mathscr{D}(G)$ is locally closed in $\mathscr{C}(G)$. If, moreover, $G$ is compactly generated, then $\mathscr{D}(G) \cap \mathscr{K}(G)$ is locally closed in $\mathscr{C}(G)$.

Proof. (i) Towards showing that the complement of $\mathscr{A}(G)$ in $\mathscr{C}(G)$ is open, we fix $C \in \mathscr{C}(G) \backslash \mathscr{A}(G)$ and $x, y \in C$ such that $x y \neq y x$. There exist neighbourhoods $U_{x}$ and $U_{y}$ of $e$ in $G$ such that, for all $x^{\prime} \in x U_{x}$ and $y^{\prime} \in y U_{y}$, we have $x^{\prime} y^{\prime} \neq y^{\prime} x^{\prime}$. Set $U=U_{x} \cap U_{y}$.

For any $D \in \mathscr{V}_{\{x, y\}, U^{-1}}(C)$ we have $\{x, y\} \subset D U^{-1}$; see (1-1). In other words, there exist $x^{\prime}, y^{\prime} \in D$ such that $x^{\prime} \in x U$ and $y^{\prime} \in y U$. In particular, $D$ is not abelian.

(ii) Suppose first that $\mathscr{K}(G)$ is open in $\mathscr{C}(G)$. Since $G$ is clearly in $\mathscr{K}(G)$, there exist a compact subset $K$ and a nonempty open subset $U$ in $G$ such that

$$
\mathscr{V}_{K, U}(G)=\{D \in \mathscr{C}(G) \mid K \subset D U\} \subset \mathscr{K}(G) .
$$

The closed subgroup $\overline{\langle K\rangle}$ generated by $K$ is in $\mathscr{V}_{K, U}(G)$, a fortiori in $\mathscr{K}(G)$. (Note that $\langle K\rangle$ need not be closed. This justifies the introduction of $\langle K, V\rangle$ below.) Let $V$ be any relatively compact open neighbourhood of $e$ in $G$. On the one hand, the subgroup $\langle K, V\rangle$ generated by $K \cup V$ is open in $G$, and therefore also closed; on the other hand, we have $\overline{\langle K\rangle} \subset\langle K, V\rangle$, and therefore $\langle K, V\rangle \subset \mathscr{K}(G)$. Thus, there exists a compact subset $L$ of $G$ such that $\langle K, V\rangle L=G$. It follows that the compact subset $K \cup \bar{V} \cup L$ generates $G$; in particular, $G$ is compactly generated.

Suppose now that $G$ is compactly generated, say by some compact subset $K$. Without loss of generality, we assume that $e \in K$ and $K^{-1}=K$. Let $V$ be a relatively compact open neighbourhood of $e$.

We fix $C \in \mathscr{K}(G)$ and choose a compact subset $L$ of $G$ such that $C L=G$. Observe that $L V$ is open in $G$; set $M=L \bar{V}$, which is also a compact subset of $G$. Since we have $M K \subset G=C L V=\bigcup_{c \in C} c L V$ with $M K$ compact and the $c L V$ 's open, there exists a finite family $c_{1}, \ldots, c_{n}$ in $C$ such that $M K \subset \bigcup_{j=1}^{n} c_{j} L V$.

By Lemma 3.2 and Remark 3.3, there exists an open neighbourhood $U$ of $e$ such that, whenever $d_{1}, \ldots, d_{n}$ in $G$ are such that $d_{j} \in c_{j} U$ for $j=1, \ldots, n$, any closed subgroup of $G$ containing $d_{1}, \ldots, d_{n}$ is cocompact. Set $F=\left\{c_{1}, \ldots, c_{n}\right\}$. Then $c_{1}, \ldots, c_{n} \in D U^{-1}$ for any closed subgroup $D \in \mathscr{V}_{F, U^{-1}}(C)$; see (1-1). In other words, any $D \in \mathscr{V}_{F, U^{-1}}(C)$ contains elements $d_{1}, \ldots, d_{n}$ such that $d_{j} \in c_{j} U$ for all $j$. We have shown that the neighbourhood $\mathscr{V}_{F, U^{-1}}(C)$ of $C$ is contained in $\mathscr{K}(G)$. This ends the proof of (ii).

(iii) Since locally compact NSS groups are metrisable (see for example [Kaplansky 1971, Chapter II, Theorem 2]), there exists a left-invariant distance function $\delta: G \times G \rightarrow \mathbf{R}_{+}$defining the topology of $G$. For a closed subgroup $C$ of $G$, define the minimum distance $\min _{\delta}(C)=\min \{\delta(e, c) \mid c \in C, c \neq e\} \in \mathbf{R}_{+}^{*} \cup\{\infty\}$. Following the standard convention, we set $\min _{\delta}(\{e\})=\infty$. It is easy to check that the mapping 
$\mathscr{C}(G) \rightarrow \mathbf{R}_{+}^{*} \cup\{\infty\}$ given by $C \mapsto \min _{\delta}(C)$ is continuous, and it follows that $\mathscr{D}(G)$ is open in $\mathscr{C}(G)$.

Remark 3.5. (i) The space $\mathscr{L}(G)$ of lattices of $G$ need not be open in $\mathscr{C}(G)$.

(ii) There are classes of groups $G$ in which all lattices are cocompact, and $\mathscr{L}(G)$ is open in $\mathscr{C}(G)$ for such groups. This applies for example to soluble Lie groups with countably many connected components; see [Raghunathan 1972, Theorem 3.1].

(iii) A locally compact group which is not unimodular does not contain any lattice [Raghunathan 1972, Remark 1.9]. In particular, for a solvable Lie group $G$, the space $\mathscr{L}(G)$ is empty "in most cases".

An example to which Remark 3.5(i) applies is the group $\mathrm{PSL}_{2}(\mathbf{R})$ of fractional linear transformations of the Poincaré half-plane $\mathscr{H}=\{z \in \mathbf{C} \mid \operatorname{Im}(z)>0\}$. For each real number $s \geq 2$, let $\Gamma_{s}$ denote the subgroup of $\mathrm{PSL}_{2}(\mathbf{R})$ generated by the transformations

$$
\left[\begin{array}{ll}
1 & s \\
0 & 1
\end{array}\right]: z \mapsto z+s \quad \text { and } \quad\left[\begin{array}{ll}
1 & 0 \\
s & 1
\end{array}\right]: z \mapsto \frac{z}{s z+1}
$$

It is standard that $\Gamma_{s}$ is a nonabelian free group of rank 2 for any $s \geq 2$, that $\Gamma_{2}$ is a lattice in $\mathrm{PSL}_{2}(\mathbf{R})$, and that $\operatorname{Vol}\left(\Gamma_{s} \backslash \mathscr{H}\right)=\infty$ for $s>2$ (so then $\Gamma_{s}$ is not a lattice); see for example [de la Harpe 2000, Exercise II.33].

Thus, in $\mathscr{C}\left(\mathrm{PSL}_{2}(\mathbf{R})\right)$, any neighbourhood of the lattice $\Gamma_{2}$ contains for $\epsilon$ small enough a nonlattice $\Gamma_{2+\epsilon}$.

Let us assume that the locally compact group $G$ is metrisable and that its topology is defined by a left-invariant distance function $\delta$ relative to which closed balls are compact. Then $\mathscr{C}(G)$ is also metrisable, and there is a basis of neighbourhoods of any point $C$ in $\mathscr{C}(G)$ that consists of the subsets

$$
\mathscr{V}_{R, \epsilon}(C)=\left\{\begin{array}{l|l}
D \in \mathscr{C}(G) & \begin{array}{l}
\delta(x, D)<\epsilon \text { for all } x \in C \cap \bar{B}_{R} \text { and } \\
\delta(y, C)<\epsilon \text { for all } y \in D \cap \bar{B}_{R}
\end{array}
\end{array}\right\} .
$$

Here, $B_{R}$ denotes for $R>0$ the open ball $\{g \in G \mid \delta(g, e)<R\}$.

There is another way to describe this topology. In $\mathscr{C}(G)$, a sequence $\left(C_{n}\right)_{n \geq 1}$ converges to $C$ if and only if

(3-4a) for any strictly increasing map $\varphi: \mathbf{N} \rightarrow \mathbf{N}$, and for any sequence $\left(g_{\varphi(n)}\right)_{n \geq 1}$ converging to some $g \in G$ with $g_{\varphi(n)} \in C_{\varphi(n)}$ for all $n \geq 1$, we have $g \in C$;

(3-4b) for any $g \in C$, there exists a sequence $\left(g_{n}\right)_{n \geq 1}$ converging to $g$ with $g_{n} \in C_{n}$ for all $n \geq 1$.

This has been observed by many authors; a proof can be found in [Benedetti and Petronio 1992, Section E.1] or in [Guivarc'h and Rémy 2006, Section 2.1]. 


\section{Closed subgroups of the group $\mathrm{C}$}

This section is an exposition of [Hubbard and Pourezza 1979]. We describe the space $\mathscr{C}(\mathbf{C})$ in four steps: First we describe the closed subspace $\mathscr{C}_{n l}(\mathbf{C})$ of closed subgroups that are not lattices and the open subspace $\mathscr{L}(\mathbf{C})$ of lattices, then we analyze the ways in which a sequence of lattices can converge to a nonlattice, and finally we give a global description of $\mathscr{C}(\mathbf{C})=\mathscr{C}_{\text {nl }}(\mathbf{C}) \cup \mathscr{L}(\mathbf{C})$.

\section{I. Closed subgroups in $\mathrm{C}$ that are not lattices.}

There is an obvious identification of the space $\mathscr{C}_{\mathbf{R}}(\mathbf{C})$ of closed subgroups of $\mathbf{C}$ isomorphic to $\mathbf{R}$ with the real projective line $\mathbf{P}^{1}$, and thus $\mathscr{C}_{\mathbf{R}}(\mathbf{C})$ is homeomorphic to a circle. Each closed subgroup $C \subset \mathbf{C}$ isomorphic to $\mathbf{Z}$ is contained in a unique group $\bar{C} \in \mathscr{C}_{\mathbf{R}}(\mathbf{C})$ and is determined by $\bar{C}$ and the "norm" $|C|:=\min \left\{|z| \in \mathbf{R}_{+}^{*}\right.$ : $z \in C, z \neq 0\}$. Correspondingly, the space $\mathscr{b}_{\mathbf{Z}}(\mathbf{C})$ is homeomorphic to the direct product of $\mathbf{P}^{1}$ with an open interval. Moreover, $C \rightarrow\{0\}$ as $|C| \rightarrow \infty$, and for $\lambda \in[0,1]$ we have $\lambda C \rightarrow \bar{C}$ as $\lambda \rightarrow 0$. Thus the space of closed subgroups of $\mathbf{C}$ isomorphic to one of $\{0\}, \mathbf{Z}$, or $\mathbf{R}$ is homeomorphic to a cone

$$
\mathscr{C}_{\mathrm{nl}}^{-}(\mathbf{C})=\left(\mathbf{P}^{1} \times[-1,0]\right) /((x,-1) \sim(y,-1)),
$$

with the vertex $(*,-1)$ corresponding to $\{0\}$, points $(x, t)$ with $-1<t<0$ corresponding to infinite cyclic groups, and the base $\mathbf{P}^{1} \times\{0\}$ corresponding to groups isomorphic to $\mathbf{R}$. (The minus sign indicates that $\mathscr{C}_{\mathrm{nl}}^{-}(\mathbf{C})$ is the lower hemisphere of a 2-sphere that plays an important role in what follows.)

A closed subgroup $C \subset \mathbf{C}$ isomorphic to $\mathbf{R} \oplus \mathbf{Z}$ has an identity component $C^{0} \in \mathscr{C}_{\mathbf{R}}(\mathbf{C})$ and is determined by $C^{0}$ and the "norm" $\min \left\{|z| \in \mathbf{R}_{+}^{*}: z \in C, z \notin C^{0}\right\}$; it is convenient to parametrise $C$ by $C^{0}$ and the inverse of this norm. This parameterisation gives a homeomorphism from $\mathscr{C}_{\mathbf{R} \oplus \mathbf{Z}}(\mathbf{C})$ to $\left.\mathbf{P}^{1} \times\right] 0,1[$. This extends to an identification of the space of closed subgroups of $\mathbf{C}$ isomorphic to one of $\mathbf{R}$, $\mathbf{R} \oplus \mathbf{Z}$, or $\mathbf{C}$ with the cone

$$
\mathscr{C}_{\mathrm{nl}}^{+}(\mathbf{C})=\left(\mathbf{P}^{1} \times[0,1]\right) /((x, 1) \sim(y, 1)),
$$

with the vertex $(*, 1)$ corresponding to $\mathbf{C}$, points $(x, t)$ with $0<t<1$ to groups isomorphic to $\mathbf{R} \oplus \mathbf{Z}$, and the base of the cone $\mathbf{P}^{1} \times\{0\}$ corresponding to groups isomorphic to $\mathbf{R}$.

By combining these observations we obtain the following proposition. This proposition is in the paper of John Hubbard and Ibrahim Pourezza [1979], but Hubbard informs us that they learned it from Adrien Douady. Moreover, it was Douady who suggested that they determine the homeomorphism type of $\mathscr{C}\left(\mathbf{R}^{2}\right)$. Apparently, this problem was of interest to Bourbaki at the time. 
Proposition 4.1. The space of closed subgroups of $\mathbf{C}$ that are not lattices is homeomorphic to a 2-sphere

$$
\mathscr{C}(\mathbf{C}) \backslash \mathscr{L}(\mathbf{C})=\mathscr{C}_{\{0\}, \mathbf{Z}, \mathbf{R}, \mathbf{R} \oplus \mathbf{Z}, \mathbf{C}}(\mathbf{C})=\mathscr{C}_{\mathrm{nl}}^{-}(\mathbf{C}) \bigcup_{\mathscr{C}_{\mathbf{R}}(\mathbf{C})} \mathscr{C}_{\mathrm{nl}}^{+}(\mathbf{C}) \approx \mathbf{S}^{2}
$$

\section{II. Lattices in $\mathrm{C}$.}

Classically, one defines for any lattice $L \subset \mathbf{C}$ two complex numbers

$$
g_{2}(L)=60 \sum_{z \in L, z \neq 0} z^{-4} \text { and } g_{3}(L)=140 \sum_{z \in L, z \neq 0} z^{-6}
$$

and one denotes by $\Delta(L)=g_{2}(L)^{3}-27 g_{3}(L)^{2}$ the discriminant of $L$. The surface

$$
\Sigma=\left\{(a, b) \in \mathbf{C}^{2} \mid a^{3}-27 b^{2}=0\right\}
$$

has an isolated singularity (cusp) at the origin and is smooth elsewhere. Set

$$
T=\Sigma \cap \mathbf{S}^{3},
$$

where $\mathbf{S}^{3}=\left\{\left.(a, b) \in \mathbf{C}^{2}|| a\right|^{2}+|b|^{2}=1\right\}$ is the unit 3-sphere. The smooth curve $T$ is a trefoil knot. It is a classical and basic result that $\Delta(L) \neq 0$ and that, moreover, the mapping

$$
\underline{g}: \mathscr{L}(\mathbf{C}) \rightarrow \mathbf{C}^{2} \backslash \Sigma, \quad L \mapsto\left(g_{2}(L), g_{3}(L)\right)
$$

is a homeomorphism. There are two classical methods of proving this. One method uses the modular function, often denoted by $J$; see for example [Saks and Zygmund 1965, Section VIII.13] or [Hurwitz 1964, Chapter II.4, Section 4]. The other method provides an explicit inverse to $g$; this associates to $(a, b) \in \mathbf{C}^{2} \backslash \Sigma$ the period lattice of the holomorphic 1-form $\overline{\mathrm{d} X} / Y$ on the genus-1 plane projective curve with equation $Y^{2} Z=4 X^{3}-a X Z^{2}-b Z^{3}$; see for example [Mumford 1976, Section 7.D].

Let $\mathscr{L}^{\text {umod }}(\mathbf{C})$ be the subspace of $\mathscr{L}(\mathbf{C})$ of unimodular lattices. The natural action of the group $\mathrm{SL}_{2}(\mathbf{R})$ on $\mathscr{L}^{\text {umod }}(\mathbf{C})$ is transitive and the isotropy subgroup of the lattice $\mathbf{Z}[i]$ is $\operatorname{SL}(2, \mathbf{Z})$; hence $\mathscr{L}^{\text {umod }}(\mathbf{C})$ is homeomorphic to $\mathrm{SL}_{2}(\mathbf{R}) / \mathrm{SL}_{2}(\mathbf{Z})$. The universal covering of this space is the universal covering $\widetilde{S L}_{2}(\mathbf{R})$ of $\mathrm{SL}_{2}(\mathbf{R})$, which is homeomorphic to an open 3-disc. It follows ${ }^{2}$ that the higher homotopy groups $\pi_{j}(\mathscr{L}(\mathbf{C}))$ for $j \geq 2$ are trivial and that $\pi_{1}(\mathscr{L}(\mathbf{C}))$ is isomorphic to the inverse image $\widetilde{S L}_{2}(Z)$ of $\mathrm{SL}_{2}(\mathbf{Z})$ in the universal covering of the group $\mathrm{SL}_{2}(\mathbf{R})$.

\footnotetext{
${ }^{2}$ This is in sharp contrast with the situation for $n \geq 3$. In that case, the universal covering of the space $\mathrm{SL}_{n}(\mathbf{R}) / \mathrm{SL}_{n}(\mathbf{Z})$ of lattices in $\mathbf{R}^{n}$ is the two-sheeted covering $\widetilde{\mathrm{SL}}_{n}(\mathbf{R})$, which is homotopic to its maximal compact subgroup $\operatorname{Spin}(n)$. In particular, $\widetilde{\mathrm{SL}}_{n}(\mathbf{R})$ is not contractible.
} 
There are two continuous actions of the multiplicative group $\mathbf{C}^{*}$ that are both natural and important in the present context:

$$
\begin{aligned}
\mathbf{C}^{*} \text { acts on } \mathscr{C}(\mathbf{C}) & \text { by } & (s, C) & \mapsto \sqrt{s} C, \\
\mathbf{C}^{*} \text { acts on } \mathbf{C}^{2} & \text { by } & (s,(a, b)) & \mapsto\left(s^{-2} a, s^{-3} b\right) .
\end{aligned}
$$

Several remarks are in order:

- The subgroup $\sqrt{s} C$ is well defined even though $\sqrt{s}$ is only defined up to a sign, since $-C=C$.

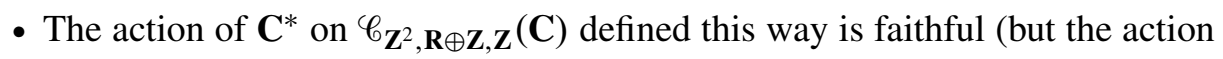
defined by $(s, C) \mapsto s C$ would not be).

- Each of the subspaces $\mathscr{L}(\mathbf{C}), \mathscr{C}_{\mathbf{R}}(\mathbf{C}), \mathscr{C}_{\mathbf{Z}}(\mathbf{C}),\{0\}, \mathscr{C}_{\mathbf{R} \oplus \mathbf{Z}}(\mathbf{C})$, and $\{\mathbf{C}\}$ of $\mathscr{C}(\mathbf{C})$ is invariant by $\mathbf{C}^{*}$.

- The action of $\mathbf{C}^{*}$ on $\mathscr{C}_{\mathbf{Z}}(\mathbf{C})$ is free and transitive.

- The hypersurface $\Sigma$ in $\mathbf{C}^{2}$ is $\mathbf{C}^{*}$-invariant.

- The mapping $\underline{g}$ of (4-2) is $\mathbf{C}^{*}$-equivariant (this carries over to $\underline{g^{\prime}}$; see (4-5) below).

The actions of the subgroups $\mathbf{R}_{+}^{*}$ (positive reals) and $\mathbf{S}^{1}=\left\{z \in \mathbf{C}^{*}|| z \mid=1\right\}$ obtained by restriction will also play a role below.

The action of $\mathbf{R}_{+}^{*}$ on $\mathscr{L}(\mathbf{C})$ is free, and its orbits are transverse to $\mathscr{L}^{\text {umod }}(\mathbf{C})$; similarly, the action of $\mathbf{R}_{+}^{*}$ on $\mathbf{C}^{2} \backslash \Sigma$ is free, and its orbits are transverse to $\mathbf{S}^{3} \backslash T$. It follows that $\mathscr{L}(\mathbf{C})$ is homeomorphic to a direct $\operatorname{product}^{3} \mathscr{L}^{\text {umod }}(\mathbf{C}) \times \mathbf{R}_{+}^{*}$, and that we have a homeomorphism

$$
\mathscr{L}^{\text {umod }}(\mathbf{C}) \approx \mathbf{S}^{3} \backslash T .
$$

In particular, the fundamental group $\widetilde{\mathrm{SL}}_{2}(\mathbf{Z})$ of the left-hand space is isomorphic to the group of the trefoil knot, also known as the Artin braid group on three strings. (The idea behind the argument leading to (4-4) is due to Daniel Quillen; see [Milnor 1971, Section 10].)

The action of $\mathbf{R}_{+}^{*}$ on $\mathscr{C}_{\mathbf{Z}}(\mathbf{C})$ is also free (as already observed), and its orbits are transverse to the subspace of subgroups of the form $\mathbf{Z} w$ with $w$ of modulus one in $\mathbf{C}$; similarly, the action of $\mathbf{R}_{+}^{*}$ on $\Sigma \backslash\{(0,0)\}$ is free, and its orbits are

\footnotetext{
${ }^{3}$ There are at least three tempting choices for a subspace $\mathscr{L}^{*}(\mathbf{C})$ that intersects every $\mathbf{R}_{+}^{*}$-orbit exactly once, so that $\mathscr{L}(\mathbf{C})$ is homeomorphic to the direct product $\mathscr{L}^{*}(\mathbf{C}) \times \mathbf{R}_{+}^{*}$ and each $\mathscr{L}^{*}(\mathbf{C})$ is homeomorphic to $\mathbf{S}^{3} \backslash T$. One choice is the space $\mathscr{L}^{\text {umod }}(\mathbf{C})$ introduced here; another choice is the space $\mathscr{L}^{\text {short=1}}(\mathbf{C})$ of lattices $L$ whose shortest vector has norm 1 , namely of lattices with $\min \{|z|: z \in L, z \neq 0\}=1$; and a third choice is the space of lattices $L$ with $\left|g_{2}(L)\right|^{2}+\left|g_{3}(L)\right|^{2}=1$. Each of these three choices has its own virtues. For example, is it apparent that there are sequences in $\mathscr{L}^{\text {umod }}(\mathbf{C})$ that converge to a subgroup isomorphic to $\mathbf{R}$, and sequences in $\mathscr{L}^{\text {short=1}}(\mathbf{C})$ that converge to subgroups isomorphic to $\mathbf{Z}$.
} 
transverse to $T$. The three spaces $\mathscr{C}_{\mathbf{Z}}(\mathbf{C}), \Sigma \backslash\{(0,0)\}$, and $T \times \mathbf{R}_{+}^{*}$ are naturally homeomorphic to each other.

An alternative description of $\mathrm{SL}_{2}(\mathbf{R}) / \mathrm{SL}_{2}(\mathbf{Z})$. For the reader familiar with the geometry of 3-manifolds, we describe another way of seeing that $\mathrm{SL}_{2}(\mathbf{R}) / \mathrm{SL}_{2}(\mathbf{Z})$, better viewed now as $\mathrm{PSL}_{2}(\mathbf{R}) / \mathrm{PSL}_{2}(\mathbf{Z})$, is homeomorphic to the complement of the trefoil knot.

The action of $\mathrm{PSL}_{2}(\mathbf{R})$ on the upper half-plane $\mathscr{H}$ by fractional linear transformations induces a simply transitive action on the unit tangent bundle $U \mathscr{H}$ (in the hyperbolic metric). Thus we may identify $\mathrm{PSL}_{2}(\mathbf{R})$ with $U \mathscr{H}$ and $\mathrm{SL}_{2}(\mathbf{R}) / \mathrm{SL}_{2}(\mathbf{Z})$ with $U \mathscr{H} / \mathrm{PSL}_{2}(\mathbf{Z})$. The latter 3-manifold is a Seifert fibre space whose base orbifold is the familiar $\mathscr{H} / \mathrm{PSL}_{2}(\mathbf{Z})$, a once punctured sphere with two cone points where the local groups are cyclic of order 2 and 3, respectively. It is well known that this Seifert fibred space is the complement of the trefoil knot.

Indeed the trefoil knot is the $(2,3)$-torus knot, and for any $p, q$ coprime, the complement in $\mathbf{S}^{3}$ of the $(p, q)$-torus $\operatorname{knot} T_{p, q}$ is a Seifert fibred space whose base orbifold is a disc with two cone points, one of order $p$ and one of order $q$. Perhaps the easiest way to see this is to observe that the nonsingular fibres of the map from $\mathbf{S}^{3} \subset \mathbf{C}^{2}$ to $\mathbf{C} \cup\{\infty\}=\mathbf{S}^{2}$ (the complex projective line) given by $\left(z_{1}, z_{2}\right) \mapsto z_{1}^{p} / z_{2}^{q}$ are $(p, q)$-torus knots; deleting one nonsingular fibre gives the structure we seek.

Returning now to the main argument, note that the mapping $\underline{g}$ of (4-2) extends to a homeomorphism

$$
\underline{g^{\prime}}: \mathscr{L}(\mathbf{C}) \cup \mathscr{C}_{\mathbf{Z}}(\mathbf{C}) \rightarrow \mathbf{C}^{2} \backslash\{(0,0)\},
$$

which is onto and $\mathbf{R}_{+}^{*}$-equivariant. For any $w \in \mathbf{C}^{*}$, we have

$$
\begin{aligned}
& g_{2}(\mathbf{Z} w)=60 \sum_{n \in \mathbf{Z}, n \neq 0}(n w)^{-4}=\frac{4 \pi^{4}}{3 w^{4}} \\
& g_{3}(\mathbf{Z} w)=140 \sum_{n \in \mathbf{Z}, n \neq 0}(n w)^{-6}=\frac{2^{3} \pi^{6}}{3^{3} w^{6}}
\end{aligned}
$$

(by an easy computation, or by [Saks and Zygmund 1965, Chapter VIII, (12.8)]). Summing up:

Proposition 4.2. With the notation above, the space of lattices in $\mathbf{C}$ is homeomorphic to $\mathbf{C}^{2} \backslash \Sigma$ or equivalently $\mathbf{R}_{+}^{*} \times\left(\mathbf{S}^{3} \backslash T\right)$. Its fundamental group is isomorphic to the trefoil knot group, and its universal covering is a 4-disc.

The homeomorphism $\mathscr{L}(\mathbf{C}) \approx \mathbf{C}^{2} \backslash \Sigma$ extends to a homeomorphism $\mathscr{b}_{\mathbf{Z}, \mathbf{Z}^{2}}(\mathbf{C}) \approx$ $\mathbf{C}^{2} \backslash\{(0,0)\}$, and further to a homeomorphism $\mathscr{C}_{\{0\}, \mathbf{Z}, \mathbf{Z}^{2}}(\mathbf{C}) \approx \mathbf{C}^{2}$. 


\section{III. Degeneration of lattices in $\mathrm{C}$.}

Let $L$ be a lattice in C. Choose $w_{1}, w_{2} \in L$ with $w_{1}$ of smallest absolute value among elements of $L \backslash\{0\}$, with $w_{2}$ of smallest absolute value among elements of $L \backslash \mathbf{Z} w_{1}$, and with $\operatorname{Im}\left(w_{2} / w_{1}\right)>0$. Then $\left(w_{1}, w_{2}\right)$ is a positively oriented $\mathbf{Z}$-basis of $L$. Define the minimal length $\ell_{1}(L):=\left|w_{1}\right|$, the second minimal length $\ell_{2}(L):=\left|w_{2}\right|$, and the distortion coefficient $\kappa(L)=\ell_{2}(L) / \ell_{1}(L)$ of $L$. Clearly, these depend on $L$ only and not on the choice of $w_{1}$ and $w_{2}$ (unless $\kappa(L)=1$, the subgroup $\mathbf{Z} w_{1}$ is also well defined). The definition of $\ell_{1}$ carries over to $\mathscr{C}_{\mathbf{Z}}(\mathbf{C})$, and that of $\ell_{2}$ (cum grano salis) to $\mathscr{C}_{\mathbf{R} \oplus \mathbf{Z}}(\mathbf{C})$. The functions

$$
\begin{aligned}
& \left.\ell_{1}: \mathscr{L}(\mathbf{C}) \cup \mathscr{C}_{\mathbf{Z}}(\mathbf{C}) \rightarrow\right] 0, \infty[, \\
& \left.\ell_{2}: \mathscr{L}(\mathbf{C}) \cup \mathscr{C}_{\mathbf{R} \oplus \mathbf{Z}}(\mathbf{C}) \rightarrow\right] 0, \infty[, \\
& \kappa: \mathscr{L}(\mathbf{C}) \rightarrow[1, \infty[
\end{aligned}
$$

are continuous. Observe that $w_{2} / w_{1}$ always belongs to the standard fundamental domain for the action of $\mathrm{PSL}_{2}(\mathbf{Z})$ on the Poincare half-plane; hence the angle spanned by $w_{1}$ and $w_{2}$ is always between $\pi / 3$ and $2 \pi / 3$.

Proposition 4.3. Let $\left(L_{n}\right)_{n \geq 1}$ be a sequence in $\mathscr{L}(\mathbf{C})$.

(i) $\lim _{n \rightarrow \infty} L_{n}=\{0\}$ if and only if $\ell_{1}\left(L_{n}\right) \rightarrow \infty$.

(ii) $\lim _{n \rightarrow \infty} L_{n}=\mathbf{C}$ if and only if $\ell_{2}\left(L_{n}\right) \rightarrow 0$.

(iii) If $\lim _{n \rightarrow \infty} L_{n}=C$ for some $C \in \mathscr{C}(\mathbf{C})$ with $C \notin \mathscr{L}(\mathbf{C}), C \neq\{0\}$, and $C \neq \mathbf{C}$, then $\kappa\left(L_{n}\right) \rightarrow \infty$.

Proof. We leave (i) and (ii) to the reader and prove (iii). We shall assume that $\kappa\left(L_{n}\right)$ does not tend to $\infty$ and reach a contradiction.

By assumption, there exists a subsequence $\left(L_{n_{k}}\right)_{k \geq 1}$ such that $\kappa\left(L_{n_{k}}\right)$ converges to some $\kappa_{0}<\infty$. Also, $\ell_{1}\left(L_{n_{k}}\right)$ is bounded above because $C \neq\{0\}$, and $\ell_{2}\left(L_{n_{k}}\right)$ is bounded below because $C \neq \mathbf{C}$; since their quotients $\kappa\left(L_{n_{k}}\right)$ are bounded, both $\ell_{1}\left(L_{n_{k}}\right)$ and $\ell_{2}\left(L_{n_{k}}\right)$ are bounded simultaneously above and below. Upon extracting a further appropriate subsequence, we can assume that $L_{n_{k}}$ contains vectors $w_{1}\left(n_{k}\right)$ and $w_{2}\left(n_{k}\right)$ defined as above, and that we have limits in $\mathbf{C}$, say $w_{1}=\lim _{k \rightarrow \infty} w_{1}\left(n_{k}\right)$ and $w_{2}=\lim _{k \rightarrow \infty} w_{2}\left(n_{k}\right)$. But then the limit group $C$ is the lattice spanned by $w_{1}$ and $w_{2}$, and this is the desired contradiction.

Our next proposition is a refinement of (iii) above; the proof is left to the reader. We denote by $[w]$ the class in $\mathbf{C}^{*} /\{ \pm \mathrm{id}\}$ of a vector $w \in \mathbf{C}^{*}$, and by $\mathbf{R} w$ its class in $\mathbf{P}^{1}$.

Proposition 4.4. Let $L_{n} \rightarrow C$ be as in the previous proposition, and let $v \in \mathbf{C}^{*}$.

(i) $C=\mathbf{Z} v$ if and only if $\kappa\left(L_{n}\right) \rightarrow \infty$ and $\left[w_{1}\left(L_{n}\right)\right] \rightarrow[v] \in \mathbf{C} /\{ \pm \mathrm{id}\}$.

(ii) $C=\mathbf{R} v \oplus \mathbf{Z}($ iv $)$ if and only if $\kappa\left(L_{n}\right) \rightarrow \infty$ and $\left[w_{2}\left(L_{n}\right)\right] \rightarrow[$ iv $] \in \mathbf{C} /\{ \pm \mathrm{id}\}$. 
(iii) $C=\mathbf{R} v$ if and only if $\ell_{1}\left(L_{n}\right) \rightarrow 0, \ell_{2}\left(L_{n}\right) \rightarrow \infty$, and $\mathbf{R} w_{1}\left(L_{n}\right) \rightarrow \mathbf{R} v \in \mathbf{P}^{1}$.

Comment on (iii). If $\ell_{1}\left(L_{n}\right)<\ell_{2}\left(L_{n}\right)$, recall that $w_{1}\left(L_{n}\right)$ is well defined up to sign, so that $\mathbf{R} w_{1}\left(L_{n}\right)$ is well defined in $\mathbf{P}^{1}$. Since $L_{n} \rightarrow C$ by hypothesis, we have $\mathbf{R} w_{1}\left(L_{n}\right) \rightarrow \mathbf{R} v$ in $\mathbf{P}^{1}$.

\section{IV. The homeomorphism $\mathscr{C}(\mathbf{C}) \approx \mathbf{S}^{\mathbf{4}}$ of [Hubbard and Pourezza 1979].}

To prepare for Theorem 4.6, which is a detailed version of Theorem 1.2, it is convenient to record the following identifications. The 4-sphere of Theorem 1.2 is identified with the one-point compactification $\mathbf{C}^{2} \cup\{\infty\}$ of $\mathbf{C}^{2}$, its north and south poles to $\infty$ and $(0,0)$ respectively, and its equator to the unit sphere

$$
\mathbf{S}^{3}=\left\{(a, b) \in \mathbf{C}^{2} \mid\|(a, b)\|=1\right\}, \quad \text { where }\|(a, b)\|=\sqrt{|a|^{2}+|b|^{2}} .
$$

We denote by $B$ the open unit ball $\left\{(a, b) \in \mathbf{C}^{2} \mid\|(a, b)\|<1\right\}$, and we denote by

$$
\gamma: \mathbf{C}^{2} \rightarrow \mathscr{C}_{\{0\}, \mathbf{Z}, \mathbf{Z}^{2}}(\mathbf{C})
$$

the inverse of the homeomorphism $g^{\prime}$ that appears in (4-5) and in Proposition 4.2.

As our aim is to define a homeomorphism $f$ from $\mathbf{C}^{2} \cup\{\infty\}$ to $\mathscr{C}(\mathbf{C})$, we will first define its restriction to $\bar{B}$. We want the image $f\left(\mathbf{S}^{3} \backslash T\right)$ to be the set of unimodular lattices; in contrast, $\gamma\left(\mathbf{S}^{3} \backslash T\right)$ contains lattices with arbitrarily large coareas (compare with note 3 ). The construction of $f$ involves several auxiliary mappings.

The coarea is traditionally defined as a function $\mathscr{L}(\mathbf{C}) \rightarrow] 0, \infty[$. We extend it by defining coarea $(C)=\infty$ if $C=\{0\}$ or $C \cong \mathbf{Z}$ and coarea $(C)=0$ if $C \cong \mathbf{R} \oplus \mathbf{Z}$ or $C=\mathbf{C}$; the resulting extension $\mathscr{C}(\mathbf{C}) \backslash \mathscr{C}_{\mathbf{R}}(\mathbf{C}) \rightarrow[0, \infty]$ is continuous. Therefore, it makes sense to define

$$
\mathscr{C}^{\text {coarea } \geq 1}(\mathbf{C})=\left\{C \in \mathscr{C}(\mathbf{C}) \backslash \mathscr{C}_{\mathbf{R}}(\mathbf{C}) \mid \operatorname{coarea}(C) \geq 1\right\},
$$

which is a subset of $\mathscr{C}_{\mathbf{Z}^{2}, \mathbf{Z},\{0\}}(\mathbf{C})$ containing $\mathscr{C}_{\mathbf{Z},\{0\}}(\mathbf{C})$.

The second auxiliary mapping is the retraction

$$
\pi: \mathbf{C}^{2} \backslash\{(0,0)\} \rightarrow \mathbf{S}^{3}, \quad(a, b) \mapsto \mathbf{R}_{+}^{*}(a, b) \cap \mathbf{S}^{3}
$$

which assigns to $(a, b)$ the intersection with $\mathbf{S}^{3}$ of its $\mathbf{R}_{+}^{*}$-orbit; see (4-3). Observe that $\pi$ is $\mathbf{S}^{1}$-equivariant (for the actions of $\mathbf{S}^{1}$ viewed as a subgroup of $\mathbf{C}^{*}$ ).

We define a third continuous mapping

$$
\left.\left.\varphi: \mathbf{C}^{2} \backslash\{(0,0)\} \rightarrow\right] 0, \infty\right], \quad(a, b) \mapsto(\operatorname{coarea}(\gamma(\pi(a, b))))^{1 / 2} .
$$

Observe that $\varphi$ is $\mathbf{C}^{*}$-invariant; also, for $(a, b) \in \mathbf{C}^{2} \backslash\{0,0\}$, we have $(a, b) \in \Sigma$ if and only if $\pi(a, b) \in T$, if and only if $\gamma(\pi(a, b)) \cong \mathbf{Z}$, and if and only if $\operatorname{coarea}(\gamma(\pi(a, b)))=\infty$. 
The fourth and last auxiliary mapping is a continuous function

$$
h: \bar{B} \rightarrow \mathbf{R}_{+} \cup\{\infty\}
$$

with the properties that

(i) $h(0,0)=0$ and

(ii) for $(a, b) \in \bar{B} \backslash\{(0,0)\}$, there exists an order-preserving homeomorphism $\eta_{\varphi(a, b)}$ from $[0,1]$ onto $[0, \varphi(a, b)]$ such that $h(a, b)=\eta_{\varphi(a, b)}(\|(a, b)\|)$.

The notation indicates that the homeomorphism $\eta_{\varphi(a, b)}$ depends on $(a, b)$ through its image by $\varphi$ only; in particular, for any $t$ and $\theta$ in $\mathbf{R}$, the homeomorphisms associated to $(a, b),\left(t^{-2} a, t^{-3} b\right)$, and $\left(e^{-2 i \theta} a, e^{-3 i \theta} b\right)$ are identical. It follows from (i) and (ii) that, for $(a, b) \in \bar{B}$,

(iii) $h(a, b)=\infty$ if and only if $(a, b) \in T$;

(iv) $h(a, b)=0$ if and only if $(a, b)=(0,0)$;

(v) $(1 / h(a, b)) \gamma(\pi(a, b)) \in \mathscr{L}^{\text {umod }}(\mathbf{C})$ if and only if $(a, b) \in \mathbf{S}^{3} \backslash T$.

The other properties of the mapping $h$ do not play any important role below. One possibility (out of many others) would be to require $\eta_{\varphi(a, b)}$ to be a fractional linear homeomorphism tangent to the identity at the origin, and therefore to set

$$
h(a, b)=\frac{\|(a, b)\|}{1+(1 / \varphi(a, b)-1)\|(a, b)\|}
$$

if $(a, b) \neq(0,0)$, and $h(0,0)=0$.

Checking that the map defined by (4-7) is continuous is equivalent to checking that the homeomorphisms $\eta_{c}:[0,1] \rightarrow[0, c]$ depend continuously on $\left.\left.c \in\right] 0, \infty\right]$ with respect to the uniform topology on the space of mappings from $[0,1]$ to the compact interval $[0, \infty]$.

For a closed subgroup $C$ of $\mathbf{C}$, we denote by

$$
\sigma(C)=\{z \in \mathbf{C} \mid \bar{z} \in C\}
$$

the complex conjugate of $C$.

Proposition 4.5. Let $f: \bar{B} \rightarrow \mathscr{C}_{\mathbf{Z}^{2}, \mathbf{R}, \mathbf{Z},\{0\}}(\mathbf{C})$ be the mapping defined by

$$
\begin{array}{llrl}
f(a, b) & =\frac{1}{h(a, b)} \gamma(\pi(a, b))=\frac{1}{\varphi(a, b)} \gamma(a, b) & & \text { for }(a, b) \in \mathbf{S}^{3} \backslash T, \\
f(a, b) & =\mathbf{R} w & & \text { for }(a, b) \in T, \\
f(a, b) & =\frac{1}{h(a, b)} \gamma(\pi(a, b)) & & \text { for }(a, b) \in B \backslash\{(0,0)\}, \\
f(0,0) & =\{0\}, &
\end{array}
$$

where $w$ is defined by $\gamma(a, b)=\mathbf{Z} w$ in the second case. 
Then $f$ is a homeomorphism of $\bar{B}$ onto $\mathscr{C}^{\text {coarea } \geq 1}(\mathbf{C}) \cup \mathscr{C}_{\mathbf{R}}(\mathbf{C})$. Moreover, $f$ is compatible with complex conjugation: $f(\bar{a}, \bar{b})=\sigma(f(a, b))$ for all $(a, b) \in \bar{B}$.

Proof. To show that $f$ is a homeomorphism, since $\bar{B}$ is compact, it is sufficient to show that $f$ is continuous, injective, and that its image is $\mathscr{C}^{\text {coarea } \geq 1}(\mathbf{C}) \cup \mathscr{C}_{\mathbf{R}}(\mathbf{C})$.

First step: $f$ is injective with image as indicated. The domain of $f$ is the disjoint union of four subsets

$$
\{(0,0)\}, \quad T, \quad \Sigma \cap(B \backslash\{(0,0)\}), \quad \bar{B} \backslash(\Sigma \cap \bar{B}) .
$$

From the definitions of $\gamma, \varphi, h$, and $f$, it is clear that $f$ maps these subsets to

$$
\{0\}, \quad \mathscr{C}_{\mathbf{R}}(\mathbf{C}), \quad \mathscr{C}_{\mathbf{Z}}(\mathbf{C}), \quad \mathscr{L}^{\text {coarea } \geq 1}(\mathbf{C}),
$$

respectively. As it is obvious that $f$ induces a bijection from the one-point subset $\{(0,0)\}$ of $\bar{B}$ onto the one-point subset $\{0\}$ of $\mathscr{C}(\mathbf{C})$, there are three more cases to check.

(i) The group $\mathbf{S}^{1}$ acts freely transitively on both $T$ and $\mathscr{C}_{\mathbf{R}}(\mathbf{C})$. Because $f$ is $\mathbf{S}^{1}$-equivariant, it follows that $f$ induces a bijection from $T$ onto $\mathscr{C}_{\mathbf{R}}(\mathbf{C})$.

(ii) Let $(a, b) \in \Sigma \cap(B \backslash\{(0,0)\})$. Then $\pi(a, b) \in T$, there exists a nonzero $w \in \mathbf{C}$ such that $f(a, b)=\mathbf{Z} w$, and $w$ is well defined up to a change of sign. Moreover, $\varphi(a, b)=\infty$ and $h(a, b)=\|(a, b)\| /(1-\|(a, b)\|)$. Thus $f$ induces a bijection from the part $\mathbf{R}_{+}^{*}(a, b) \cap B$ in $B$ of the $\mathbf{R}_{+}^{*}$-orbit of $(a, b)$ onto the set of infinite cyclic subgroups of $\mathbf{C}$ contained in $\mathbf{R} w$. It follows that $f$ induces a bijection from $\Sigma \cap(B \backslash\{(0,0)\})$ onto $\mathscr{C}_{\mathbf{Z}}(\mathbf{C})$.

(iii) Let $(a, b) \in \bar{B} \backslash(\Sigma \cap \bar{B})$. Then $\pi(a, b) \in \mathbf{S}^{3} \backslash T$ and $L_{0}:=\gamma(\pi(a, b)) \in \mathscr{L}(\mathbf{C})$. Thus $f$ induces a bijection from $\mathbf{R}_{+}^{*}(a, b) \cap \bar{B}$ onto the set of lattices of the form $(1 / t) L_{0}$ with $0<t \leq\left(\operatorname{coarea}\left(L_{0}\right)\right)^{1 / 2}$, namely, with coarea $\left((1 / t) L_{0}\right) \geq 1$. It follows that $f$ induces a bijection from $\bar{B} \backslash(\Sigma \cap \bar{B})$ onto $\mathscr{L}^{\text {coarea } \geq 1}(\mathbf{C})$.

Second step: $f$ is continuous. It is clear that $f$ is continuous at any point of $\bar{B} \backslash(T \cup\{(0,0)\})$, because the mappings $\gamma, \pi, \varphi$, and $h$ are continuous (and, moreover, $h(a, b)<\infty)$. It remains to check the continuity of $f$ first at the origin and at the points of $T$.

(i) To show that $f$ is continuous at $(0,0)$, it is enough by Proposition 4.3(i) to show that $\ell_{1}(f(a, b)) \rightarrow \infty$ when $(a, b) \rightarrow(0,0)$. Since the function

$$
\mathbf{S}^{3} \rightarrow \mathbf{R}_{+}^{*}, \quad(a, b) \mapsto \ell_{1}(\gamma(a, b))
$$

is continuous and positive on a compact domain, its minimum is positive. Since $\lim _{(a, b) \rightarrow(0,0)} h(a, b)=0$, we have

$$
\ell_{1}(f(a, b))=\frac{1}{h(a, b)} \ell_{1}(\gamma(\pi(a, b))) \rightarrow \infty \quad \text { if }(a, b) \rightarrow(0,0) .
$$


Thus $f$ is indeed continuous at the origin.

(ii) Let $\left(a_{0}, b_{0}\right) \in T$. If $(a, b)$ tends to $\left(a_{0}, b_{0}\right)$ inside $T$, then $f(a, b) \cong \mathbf{R}$ tends to $f\left(a_{0}, b_{0}\right) \cong \mathbf{R}$ by the continuity of the $\mathbf{S}^{1}$-action (which is transitive on both $T$ and $\left.\mathscr{C}_{\mathbf{R}}(\mathbf{C})\right)$ and the $\mathbf{S}^{1}$-equivariance of $f$. If $(a, b)$ tends to $\left(a_{0}, b_{0}\right)$ inside $\Sigma \cap B$, it is a straightforward consequence of the definition of $f$ and of the continuity of $h$ that $f(a, b) \cong \mathbf{Z}$ tends to $f\left(a_{0}, b_{0}\right) \cong \mathbf{R}$. Therefore, we can assume from now on that $(a, b)$ tends to $\left(a_{0}, b_{0}\right)$ inside $\bar{B} \backslash(\Sigma \cap \bar{B})$, and we have to show that $f(a, b) \cong \mathbf{Z}^{2}$ tends to $f\left(a_{0}, b_{0}\right) \cong \mathbf{R}$; for this, we are going to check that the three conditions of Proposition 4.4(iii) are satisfied.

We have $\ell_{1}(f(a, b))=\ell_{1}(\gamma(\pi(a, b))) / h(a, b)$. The function $\ell_{1} \circ \gamma$ is continuous on $\mathbf{S}^{3}$, so $\ell_{1}(\gamma(\pi(a, b)))$ is bounded, and $h(a, b) \rightarrow \infty$. Hence $\ell_{1}(f(a, b)) \rightarrow 0$ when $(a, b) \rightarrow\left(a_{0}, b_{0}\right)$.

We have $1 \leq \operatorname{coarea}(f(a, b)) \leq \ell_{1}(f(a, b)) \ell_{2}(f(a, b))$. The previous point implies that $\ell_{2}(f(a, b)) \rightarrow \infty$ when $(a, b) \rightarrow\left(a_{0}, b_{0}\right)$.

For points $(a, b)$ inside $\bar{B} \backslash(\Sigma \cap \bar{B})$ and near enough to $\left(a_{0}, b_{0}\right)$, we have $\ell_{1}(\gamma(a, b))<\ell_{2}(\gamma(a, b))$, so $\mathbf{R} w_{1}(\gamma(a, b))$ is well defined in $\mathbf{P}^{1}$; moreover

$$
\mathbf{R} w_{1}(\gamma(a, b))=\mathbf{R} w_{1}(\gamma(\pi(a, b)))=\mathbf{R} w_{1}(f(a, b)) .
$$

Since the mappings $\gamma$ and $(a, b) \mapsto \mathbf{R} w_{1}(\gamma(a, b))$ are continuous in appropriate domains, we have $\mathbf{R} w_{1}(f(a, b)) \rightarrow \mathbf{R} v$.

Third step. For each $C \in \mathscr{L}(\mathbf{C}) \cup \mathscr{C}_{\mathbf{Z}}(\mathbf{C})$ we have $g_{2}(\sigma(C))=\overline{g_{2}(C)}$, and similarly for $g_{3}$. It follows that $f(\bar{a}, \bar{b})=\sigma(f(a, b))$ for all $(a, b) \in \bar{B}$. We leave the details to the reader.

We can now state the final result of this expository section. We denote by $\delta$ the inversion of $\mathbf{C}^{2} \cup\{\infty\}$ that exchanges $(0,0)$ and $\infty$ and that is defined on other points by $\delta(a, b)=(a, b) /\|(a, b)\|^{2}$. For a closed subgroup $C$ of $\mathbf{C}$, we denote by

$$
C^{*}=\{z \in \mathbf{C} \mid \operatorname{Im}(\bar{z} w) \in \mathbf{Z} \text { for all } w \in C\}
$$

the dual of $C$. It is straightforward to check that this duality is a homeomorphism that exchanges the following pairs of groups:

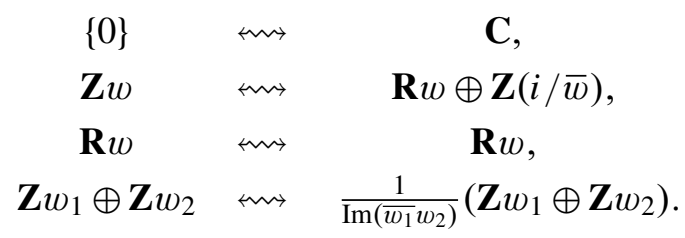

In particular, its fixed point set in $\mathscr{C}(\mathbf{C})$ is exactly the image $\mathscr{L}^{\text {umod }}(\mathbf{C}) \cup \mathscr{C}_{\mathbf{R}}(\mathbf{C})$ by $f$ of the fixed point set $\mathbf{S}^{3}$ of the inversion $\delta$. The main result of this section (and of [Hubbard and Pourezza 1979]) follows: 
Theorem 4.6. Let $f: \mathbf{C}^{2} \cup\{\infty\} \rightarrow \mathscr{b}(\mathbf{C})$ be the extension of the mapping $f$ of Proposition 4.5 defined by $f(a, b)=f(\delta(a, b))^{*}$ when $(a, b) \notin \bar{B}$. Then $f$ is $a$ homeomorphism from $\mathbf{C}^{2} \cup\{\infty\}$, which is homeomorphic to $\mathbf{S}^{4}$, to the space $\mathscr{C}(\mathbf{C})$ of closed subgroups of $\mathbf{C}$, equipped with the Chabauty topology. Moreover, $f$ has the following properties:

(i) The images of the subspaces

$\mathbf{C}^{2} \backslash \Sigma, \quad \mathbf{S}^{3} \backslash T, \quad T, \quad(\Sigma \cap B) \backslash\{(0,0)\}, \quad\{(0,0)\}, \quad \Sigma \cap\left(\mathbf{C}^{2} \backslash \bar{B}\right), \quad\{\infty\}$ of $\mathbf{C}^{2} \cup\{\infty\}$ are, respectively, these subspaces of $\mathscr{C}(\mathbf{C})$ :

$\mathscr{L}(\mathbf{C}), \quad \mathscr{L}^{\text {umod }}(\mathbf{C}), \quad \mathscr{C}_{\mathbf{R}}(\mathbf{C}), \quad \mathscr{C}_{\mathbf{Z}}(\mathbf{C}), \quad\{0\}, \quad \mathscr{C}_{\mathbf{R} \oplus \mathbf{Z}}(\mathbf{C}), \quad\{\mathbf{C}\}$.

(ii) Inversion in $\mathbf{C}^{2}$ corresponds to duality in $\mathscr{C}(\mathbf{C})$ :

$$
f(\delta(a, b))=f(a, b)^{*} \quad \text { for all }(a, b) \in \mathbf{C}^{2} \cup\{\infty\} .
$$

(iii) For the action of $\mathbf{S}^{1}$ defined by (4-3), the mapping $f$ is equivariant:

$$
f\left(e^{-i 2 \theta} a, e^{-i 3 \theta} b\right)=e^{i \theta / 2} f(a, b) \quad \text { for all }(a, b) \in \mathbf{C}^{2} \text { and } \theta \in \mathbf{R} .
$$

(iv) The homeomorphism $f$ is compatible with complex conjugation:

$$
f(\bar{a}, \bar{b})=\sigma(f(a, b)) \quad \text { for all }(a, b) \in \mathbf{C}^{2} \cup\{\infty\} .
$$

(v) For the action of $\mathbf{R}_{+}^{*}$ defined by (4-3) and for $(a, b) \in \mathbf{C}^{2} \backslash \Sigma$, the lattices $f(a, b)$ and $\underline{g}^{-1}(a, b)$, see (4-2), are in the same $\mathbf{R}_{+}^{*}$-orbit.

\section{Generalities on the real Heisenberg group}

The rest of this article is dedicated to the study of the 3-dimensional Heisenberg group $H$ and its subgroups.

We begin by recalling a few basic properties of $H$. Its centre $Z(H)$ can be identified with the second factor $\mathbf{R}$ in the description $H=\mathbf{C} \times \mathbf{R}$ of (1-3); moreover, $Z(H)$ coincides with the commutator subgroup $[H, H]$. There is a canonical projection

$$
p: H \rightarrow H / Z(H)=\mathbf{C},
$$

as in (1-3), and $H$ is an extension of $\mathbf{C}$ by $\mathbf{R}$ which does not split (unlike the extension corresponding to Equation (1-4)). It is useful to have the formula for commutators

$$
(z, t)\left(z^{\prime}, t^{\prime}\right)(z, t)^{-1}\left(z^{\prime}, t^{\prime}\right)^{-1}=\left(0, \operatorname{Im}\left(\bar{z} z^{\prime}\right)\right) .
$$


We denote by $\operatorname{Aut}(H)$ the group of continuous ${ }^{4}$ automorphisms of $H$. Any $\Phi \in \operatorname{Aut}(H)$ preserves the center and thus descends to a continuous automorphism of $H / Z(H) \cong \mathbf{R}^{2}$. Hence we have a homomorphism

$$
\Pi: \operatorname{Aut}(H) \rightarrow \mathrm{GL}_{2}(\mathbf{R}) .
$$

This is onto. The inner automorphisms act on $H$ by

$$
\operatorname{Int}_{(w, *)}(z, t)=(w, *)(z, t)(w, *)^{-1}=(z, t+\operatorname{Im}(\bar{w} z)),
$$

where $\operatorname{Int}_{(w, *)}$ is written for $\operatorname{Int}_{(w, s)}$, with $s$ arbitrary; these automorphisms form a normal subgroup $\operatorname{Int}(H)$ of $\operatorname{Aut}(H)$ isomorphic to $H / Z(H) \cong \mathbf{R}^{2}$. It follows from (5-4) that $\operatorname{Int}(H) \subset \operatorname{ker}(\Pi)$, so that we have a sequence

$$
\{1\} \rightarrow \operatorname{Int}(H) \rightarrow \operatorname{Aut}(H) \stackrel{\Pi}{\longrightarrow} \mathrm{GL}_{2}(\mathbf{R}) \rightarrow\{1\} .
$$

Proposition 5.1. The sequence (5-5) is exact and split, so that the group of continuous automorphisms of $H$ is a semidirect product

$$
\operatorname{Aut}(H)=\operatorname{Int}(H) \rtimes \mathrm{GL}_{2}(\mathbf{R}) \cong \mathbf{R}^{2} \rtimes \mathrm{GL}_{2}(\mathbf{R}) .
$$

In particular, in its natural topology, $\operatorname{Aut}(H)$ has two connected components.

Note. We write $\mathrm{GL}_{2}(\mathbf{R})$ on the right side of $\operatorname{Int}(H) \rtimes \mathrm{GL}_{2}(\mathbf{R})$, but nevertheless we regard $\mathrm{GL}_{2}(\mathbf{R})$ as acting from the left on $\mathbf{R}^{2} \cong \operatorname{Int}(H)$.

Proof. The group $\operatorname{Aut}(H)$ contains a copy of $\mathrm{GL}_{2}(\mathbf{R})$, acting by

$$
\left(\begin{array}{ll}
a & b \\
c & d
\end{array}\right)(x+i y, t)=((a x+b y)+i(c x+d y),(a d-b c) t) .
$$

Consequently, the homomorphism $\Pi$ in (5-5) has a section. As we have already observed that $\operatorname{Int}(H) \subset \operatorname{ker}(\Pi)$, it remains to justify the opposite inclusion.

Let $\Phi \in \operatorname{ker}(\Pi)$. Since $\Phi$ acts as the identity on $H / Z(H)$, it is of the form

$$
(x+i y, t) \mapsto(x+i y, t+\varphi(x+i y, t))
$$

for some mapping $\varphi: H \rightarrow \mathbf{R}$. The multiplication identity

$$
\Phi(x+i y, t) \Phi\left(x^{\prime}+i y^{\prime}, t^{\prime}\right)=\Phi\left((x+i y, t)\left(x^{\prime}+i y^{\prime}, t^{\prime}\right)\right)
$$

reduces to $\varphi(x+i y, t)+\varphi\left(x^{\prime}+i y^{\prime}, t^{\prime}\right)=\varphi\left((x+i y, t)\left(x^{\prime}+i y^{\prime}, t^{\prime}\right)\right)$. Thus, $\varphi$ is a continuous group homomorphism with values in the abelian group $\mathbf{R}$. It follows that $\varphi$ factors through a homomorphism $H /[H, H] \cong \mathbf{R}^{2} \rightarrow \mathbf{R}$, namely a linear form on $\mathbf{R}^{2}$, say $(x, y) \mapsto-v x+u y$ for some $u, v \in \mathbf{R}$. If $w=u+i v$, it follows from (5-4) that $\Phi=\operatorname{Int}_{w, *} \in \operatorname{Int}(H)$.

\footnotetext{
${ }^{4}$ Any automorphism of $H$ happens to be continuous, but this fact does not play any role in our presentation.
} 
We record here a few more observations concerning $\operatorname{Aut}(H)$.

- The centre $Z(H)$ is fixed elementwise by the derived group of $\operatorname{Aut}(H)$, namely by $\mathbf{R}^{2} \rtimes \mathrm{SL}_{2}(\mathbf{R})$.

- The projection $p$ of (5-1) is $\mathrm{GL}_{2}(\mathbf{R})$-equivariant, where the action on $\mathbf{C}$ is the standard action.

- The simple group $\mathrm{SL}_{2}(\mathbf{R})$ is a Levi factor in the identity component of the group $\operatorname{Aut}(H)$. There are many choices for such a factor, and each one corresponds to a choice of a "supplement" $\mathbf{C}$ to the centre as one writes $H=\mathbf{C} \times \mathbf{R}$; indeed, once the Levi factor $\mathrm{SL}_{2}(\mathbf{R})$ is chosen, the slices $\mathbf{C}^{*} \times\left\{t_{0}\right\}$ are the $\mathrm{SL}_{2}(\mathbf{R})$-orbits in $H$.

Proposition 5.2. (i) Let $C$ be a closed subgroup of $H$ such that $C \cap Z(H) \neq\{e\}$. Then the subgroup $p(C)$ of $\mathbf{C}$ is closed. In particular, if $C$ is any nonabelian closed subgroup of $H$, then $p(C)$ is closed.

(ii) Let $C$ be a nonabelian closed subgroup of $H$. Then, either $C$ contains $Z(H)$ or $C$ is a lattice in $H$. In other words, $\mathscr{C}(H)=\mathscr{L}(H) \cup \mathscr{A}(H) \cup \mathscr{C}_{\geq Z}(H)$.

(iii) The assignment $C \mapsto p(C)$ defines a continuous map

$$
p_{*}: \mathscr{C}(H) \backslash \mathscr{A}(H) \rightarrow \mathscr{C}(\mathbf{C}) .
$$

(iv) The spaces $\mathscr{L}(H)$ and $\mathscr{L}_{! !}(H)$ are open in $\mathscr{C}(H)$.

(v) The assignment $C \mapsto C \cap Z(H)$ defines a continuous map

$$
\mathscr{b}(H) \backslash \mathscr{A}(H) \rightarrow \mathscr{C}(Z(H)) .
$$

Remark. In (i), the condition that $C$ is nonabelian cannot be removed. Consider for example the subgroup $A_{\theta}$ in $H=\mathbf{C} \times \mathbf{R}$ generated by $(\theta, 1)$ and $(1,1)$, where $\theta$ is an irrational real number. This subgroup is closed, isomorphic to $\mathbf{Z}^{2}$, and its projection in $\mathbf{C}$ is dense in a real line.

Similarly, the map of (v) is not continuous on the whole of $\mathscr{C}(H)$.

The map $p_{*}$ of (iii) doesn't extend continuously on $\mathscr{C}(H)$; see Proposition 8.8.

Proof. (i) Let $\left(z_{n}\right)_{n \geq 1}$ be a sequence in $p(C)$ that converges to a limit $z \in \mathbf{C}$. There exists a sequence $\left(t_{n}\right)_{n \geq 1}$ in $\mathbf{R}$ such that $\left(z_{n}, t_{n}\right) \in C$ for all $n \geq 1$. Upon multiplying each $\left(z_{n}, t_{n}\right)$ by $\left(0, t_{n}^{\prime}\right)$ for appropriate $t_{n}^{\prime} \in C \cap Z(H)$, we can assume that the sequence $\left(t_{n}\right)_{n \geq 1}$ is bounded; upon extracting a subsequence, we can assume that the sequence $\left(t_{n}\right)_{n \geq 1}$ is convergent in $\mathbf{R}$. It follows that the sequence $\left(\left(z_{n}, t_{n}\right)\right)_{n \geq 1}$ converges to some element $(z, t) \in C$ with $z=p(z, t) \in p(C)$.

The last claim of (i) follows from the fact that, whenever $C$ contains two noncommuting elements, their commutator is in $(C \cap Z(H)) \backslash\{e\}$. 
(ii) Let $C$ be in $\mathscr{C}(H) \backslash \mathscr{A}(H)$, so $p(C) \in \mathscr{C}_{\mathbf{Z}} \mathbf{Z}^{2} \mathbf{R} \oplus \mathbf{Z}, \mathbf{C}(\mathbf{C})$. Recall from Subsection 4.IV that the coarea of $p(C)$ is well defined and lies in $[0, \infty[$. We distinguish two cases.

In the first case, coarea $(p(C))=0$. For every $\epsilon>0$, there exist two noncommuting elements $(z, t),\left(z^{\prime}, t^{\prime}\right) \in C$ such that $0<\left|\operatorname{Im}\left(\bar{z} z^{\prime}\right)\right|<\epsilon$. The commutator of these two elements is $\left(0, \operatorname{Im}\left(\bar{z} z^{\prime}\right)\right) \in C \cap Z(H)$. Since $C$ is closed, it follows that $Z(H) \subset C$.

In the second case, coarea $(p(C))>0$. Now $p(C) \in \mathscr{L}(\mathbf{C})$, and by Equation (5-2) the commutator group $[C, C]$ is generated by $(0$, coarea $(p(C)))$. If $C \cap Z(H)$ is not the whole of $Z(H) \approx \mathbf{R}$ then, being closed, it must be cyclic (hence discrete) and contain $[C, C]$ as a subgroup of finite index, $n$ say, in which case $C \in \mathscr{L}_{n}(H)$.

(iii) Let $\left(C_{n}\right)_{n \geq 1}$ be a sequence converging to some $C_{0}$ in $\mathscr{C}(H) \backslash \mathscr{A}(H)$. By (i), $p\left(C_{n}\right)$ is a closed subgroup of $\mathbf{C}$ for all $n \geq 1$. We have to show that $\left(p\left(C_{n}\right)\right)_{n \geq 1}$ converges to $p\left(C_{0}\right)$.

Let $\varphi: \mathbf{N} \rightarrow \mathbf{N}$ be a strictly increasing map and let $\left(y_{\varphi(n)}\right)_{n \geq 1}$ be a sequence in $\mathbf{C}$ converging to some $y \in \mathbf{C}$, with $y_{\varphi(n)} \in p\left(C_{\varphi(n)}\right)$ for all $n \geq 1$. For $n \geq 1$, choose $x_{\varphi(n)} \in C_{\varphi(n)}$ such that $p\left(x_{\varphi(n)}\right)=y_{\varphi(n)}$. Upon multiplying $x_{\varphi(n)}$ by an appropriate element in $C_{\varphi(n)} \cap Z(H)$, see claim (ii), we can assume that the sequence $\left(x_{\varphi(n)}\right)_{n \geq 1}$ is bounded in $H$, and therefore has a subsequence that converges to some $x \in H$. As $\left(C_{n}\right)_{n \geq 1}$ converges to $C_{0}$, we have $x \in C$, and therefore $y=p(x) \in p(C)$. Thus condition (3-4a) applies.

It is straightforward to check condition (3-4b).

(iv) We know from Remark 3.5(ii) that $\mathscr{L}(H)$ is open in $\mathscr{C}(H)$. (And we also know that $\mathscr{L}(H)$ is nonempty; more on this in Section 7.)

Consider $\mathscr{L}_{! !}(H)=\{C \in \mathscr{C}(H) \backslash \mathscr{A}(H) \mid p(C) \in \mathscr{L}(\mathbf{C})\}$. It follows from (iii) and the openness of $\mathscr{L}(\mathbf{C})$ in $\mathscr{C}(\mathbf{C})$ that $\mathscr{L}_{! !}(H)$ is open in $\mathscr{C}(H) \backslash \mathscr{A}(H)$. As the latter space is open in $\mathscr{C}(H)$, by Proposition $3.4, \mathscr{L}_{! !}(H)$ is also open in $\mathscr{C}(H)$.

(v) Let $C_{n} \rightarrow C_{0}$ be as in the beginning of the proof of (iii). We have to show that $\left(C_{n} \cap Z(H)\right)_{n \geq 1}$ converges to $C_{0} \cap Z(H)$. Since condition (3-4a) is straightforward to check, we need only verify condition (3-4b). Let $t \in C_{0} \cap Z(H)$; we have to find $t_{k} \in C_{k} \cap Z(H)$ so that $\lim _{k \rightarrow \infty} t_{k}=t$.

Choose $u, v \in C_{0}$ such that $[u, v] \neq e$. For all $k \geq 1$, choose also $g_{k}, u_{k}, v_{k} \in$ $C_{k}$ such that $\lim _{k \rightarrow \infty} g_{k}=t, \lim _{k \rightarrow \infty} u_{k}=u$, and $\lim _{k \rightarrow \infty} v_{k}=v$. Observe that $\lim _{k \rightarrow \infty} p\left(g_{k}\right)=0$; also, $p(u)$ and $p(v)$ are $\mathbf{R}$-linearly independent (since $[u, v] \neq e$ ), so $p\left(u_{k}\right)$ and $p\left(v_{k}\right)$ are $\mathbf{R}$-linearly independent for $k$ large enough. Set $I=\left\{k \geq 1 \mid g_{k} \notin C_{k} \cap Z(H)\right\}$. In case $I$ is finite, we can set $t_{k}=g_{k}$ if $k \notin I$ and choose $t_{k}$ arbitrarily if $t \in I$. From now on, for convenience, we assume that $I$ is an infinite set of integers. 
As $p\left(g_{k}\right) \neq 0$ and $\lim _{k \in I, k \rightarrow \infty} p\left(g_{k}\right)=0$, it follows that the coarea of the lattice generated in $\mathbf{C}$ by $p\left(g_{k}\right), p\left(u_{k}\right), p\left(v_{k}\right)$ tends to 0 , and therefore that $C_{k} \cap Z(H)$ is more and more dense in $Z(H)$ when $k \in I, k \rightarrow \infty$. Thus we can assure that $\lim _{k \rightarrow \infty} t_{k}=t$ by defining $t_{k}=g_{k}$ when $k \notin I$ and choosing $t_{k} \in C_{k} \cap Z(H)$ sufficiently close to $g_{k}$ when $k \in I$.

Let $\Lambda$ be a nonabelian closed subgroup of $H$ that does not contain $Z(H)$. It follows from Proposition 5.2 that $\Lambda$ is a lattice; moreover, the commutator subgroup $[\Lambda, \Lambda]$ is of finite index in the centre $Z(\Lambda)=\Lambda \cap Z(H)$ of $\Lambda$. For each integer $n \geq 1$, recall from Section 1 that we denote by $\mathscr{L}_{n}(H)$ the space of lattices $\Lambda$ such that $[Z(\Lambda):[\Lambda, \Lambda]]=n$. We define

$$
p_{n}: \mathscr{L}_{n}(H) \rightarrow \mathscr{L}(\mathbf{C})
$$

to be the projection induced by $p$.

Proposition 5.3. (i) For any $n \geq 1$, the subspace $\mathscr{L}_{n}(H)$ is open in $\mathscr{C}(H)$.

(ii) For $(z, t),\left(z^{\prime}, t^{\prime}\right) \in H$, if $z$ and $z^{\prime}$ generate a lattice in $\mathbf{C}$, then $(z, t)$ and $\left(z^{\prime}, t^{\prime}\right)$ generate a lattice in $H$.

Proof. (i) The function $J: \mathscr{L}_{! !}(H) \rightarrow \mathbf{R}_{+}$defined by

$$
J(D)= \begin{cases}1 / n & \text { if } D \in \mathscr{L}_{n}(H) \\ 0 & \text { if } D \in \mathscr{L}_{\infty}(H)\end{cases}
$$

is continuous because

$$
J(D)=\min \{|t| \mid t \in D \cap Z(H), t \neq e\} / \operatorname{coarea}(p(D))
$$

and each of the functions

$$
\begin{aligned}
\mathscr{L}_{! !}(H) & \rightarrow \mathscr{C}(Z(H)), & & D \mapsto D \cap Z(H), \\
\mathscr{C}_{\mathbf{Z}, \mathbf{R}}(Z(H)) & \rightarrow \mathbf{R}_{+}, & & D \mapsto \inf \{|t| \mid t \in D, t \neq e\}, \\
\mathscr{L}(\mathbf{C}) & \rightarrow \mathbf{R}_{+}^{*}, & & L \mapsto \operatorname{coarea}(L)
\end{aligned}
$$

is continuous.

Thus $\mathscr{L}_{n}(H)$ is open in $\mathscr{L}(H)$ since it is the inverse image of the point $1 / n$, which is open in the image of $J$. As $\mathscr{L}(H)$ is open in $\mathscr{C}(H)$, the space $\mathscr{L}_{n}(H)$ is also open in $\mathscr{C}(H)$.

(ii) Denote by $\Gamma$ the subgroup of $H$ generated by two elements $a=(z, t)$ and $b=\left(z^{\prime}, t^{\prime}\right)$, and set $\Lambda=p(\Gamma)$. Since $H$ is nilpotent of class two, each element $\gamma \in \Gamma$ can be written as $\gamma=a^{q} b^{r}[a, b]^{s}$, where $q, r, s \in \mathbf{Z}$.

Assume that $\Lambda$ is a lattice in C. Then $p(\gamma)=q z+r z^{\prime}=0$ if and only if $q=r=0$, and therefore $\Gamma \cap \operatorname{ker}(p)$ is the infinite cyclic group generated by 
$[a, b]=\left(0, \operatorname{Im}\left(\bar{z} z^{\prime}\right)\right)$. Since $p(\Gamma)=\Lambda$ and $\Gamma \cap \operatorname{ker}(p)=\Gamma \cap Z(H)$ are lattices in $\mathbf{C}$ and $Z(H)$ respectively, $\Gamma$ is a lattice in $H$.

\section{Closed subgroups of the Heisenberg group which are not lattices}

Our analysis of $\mathscr{C}(H)$ is spread over the next three sections. In the present section we describe the subspace formed by the closed subgroups that are not lattices. In Subsections 6.I and 6.II, we deal with closed subgroups that have Zariski closures of dimension 1 and 2, respectively; observe that, since $H$ is torsion-free, the trivial group $\{e\}$ is the only subgroup with Zariski closure of dimension 0. In 6.III, we consider the subgroups which contain the centre $Z(H)$; some, but not all, appear already in Subsections 6.I and 6.II. In 6.IV, we describe the action of the group $\operatorname{Aut}(H)$ on the nonlattice part of $\mathscr{C}(H)$.

\section{I. Groups isomorphic to $R$ and $Z$.}

(I.i) One-parameter subgroups in $H$. Any such subgroup is a real line through the origin in $\mathbf{C} \times \mathbf{R}$, that is, it is of the form

$$
\left\{\left(s z_{0}, s t_{0}\right) \in H \mid s \in \mathbf{R}\right\} \quad \text { for some }\left(z_{0}, t_{0}\right) \in H \text { with }\left(z_{0}, t_{0}\right) \neq(0,0) .
$$

The centre $Z(H)$ corresponds to $z_{0}=0$. In the Chabauty topology, these groups constitute a space

$$
\mathscr{C}_{\mathbf{R}}(H) \approx \mathbf{P}^{2}
$$

that is homeomorphic to a real projective plane.

(I.ii) Infinite cyclic subgroups. Any $h \neq e$ in $H$ generates a closed subgroup isomorphic to $\mathbf{Z}$, and each of these subgroups has exactly two generators. Thus the subgroups isomorphic to $\mathbf{Z}$ constitute a space

$$
\left.\mathscr{C}_{\mathbf{Z}}(H)=(H \backslash\{e\}) /\{\mathrm{id}, J\} \approx \mathbf{P}^{2} \times\right] 0, \infty[
$$

homeomorphic to the direct product of a real projective plane and an open interval. Here $J$ denotes the antiautomorphism of $H$ mapping each element $h=(z, t)$ to its inverse $h^{-1}=(-z,-t)$.

(I.iii) The closure of the space of cyclic subgroups. The closure in $\mathscr{C}(H)$ of $\mathscr{C}_{\mathbf{Z}}(H)$ is the space of subgroups that are of type (0), (I.i) or (I.ii). It is a closed cone on a projective plane

$$
\mathscr{C}_{\{0\}, \mathbf{Z}, \mathbf{R}}(H) \approx\left(\mathbf{P}^{2} \times[0, \infty]\right) /((x, 0) \sim(y, 0)),
$$

with the vertex $(*, 0)$ of the cone corresponding to $\{e\}$, the points in $\left.\mathbf{P}^{2} \times\right] 0, \infty[$ corresponding to infinite cyclic subgroups, and the points in $\mathbf{P}^{2} \times\{\infty\}$ to one parameter subgroups. 


\section{II. Other abelian groups: those isomorphic to $R^{2}, R \oplus Z$, and $Z^{2}$.}

(II.i) Maximal abelian subgroups. The multiplication formula (1-3) shows that the maximal abelian subgroups of $H$ are of the form

$$
\left\{\left(s z_{0}, t\right) \in H \mid(s, t) \in \mathbf{R}^{2}\right\} \text { for some } z_{0} \in \mathbf{C}^{*} ;
$$

they are all isomorphic to $\mathbf{R}^{2}$. There is an obvious homeomorphism from the space of such subgroups to the space of real lines through the origin in $\mathbf{C}$, that is,

$$
\mathscr{C}_{\mathbf{R}^{2}}(H) \approx \mathbf{P}^{1} .
$$

Note that every maximal abelian subgroup of $H$ contains the centre $Z(H) \cong \mathbf{R}$.

It is convenient to fix a left-invariant Riemannian metric on $H$ for which the submanifold $\mathbf{C} \times\{0\}$ of $H$ (caveat: it is not a subgroup of $H$ !) is orthogonal to the centre $\{0\} \times \mathbf{R}=Z(H)$. The induced Riemannian metric on each $A \in \mathscr{C}_{\mathbf{R}^{2}}(H)$ makes it isometric to the Euclidean plane $\mathbf{R}^{2}$. There are two isometric isomorphisms $A \rightarrow \mathbf{C}$ extending the isomorphism $Z(H) \rightarrow \mathbf{R}$ implicit in the notation $\{0\} \times \mathbf{R}=Z(H)$; these correspond to a choice of orientation on $A$. We define $\widehat{\mathscr{C}}_{\mathbf{R}^{2}}(H)$ to be the set of such groups $A$ together with an orientation (equivalently, choice of isomorphism to $\mathbf{C})$. There is an obvious bijection from $\widehat{\mathscr{C}}_{\mathbf{R}^{2}}(H)$ to the circle $\mathbf{S}^{1}$ of unit vectors in the slice $\mathbf{C} \times\{0\}$ of $H=\mathbf{C} \times \mathbf{R}$. The change of orientation map defines a free $(\mathbf{Z} / 2 \mathbf{Z})$-action on $\widehat{\mathscr{C}}_{\mathbf{R}^{2}}(H)$, and we have a two-sheeted covering

$$
\mathbf{S}^{1} \approx \widehat{\mathscr{C}}_{\mathbf{R}^{2}}(H) \rightarrow \mathscr{C}_{\mathbf{R}^{2}}(H) \approx \mathbf{P}^{1}
$$

which, tracing through the identifications, is the standard degree-two covering of $\mathbf{P}^{1}$ (see the remark just before Theorem 1.3).

Notation. Any closed abelian subgroup $A$ of $H$ that is not contained in $Z(H)$ is contained in a unique closed subgroup of $H$ isomorphic to $\mathbf{R}^{2}$, and we shall denote this by $\bar{A}$. When we place a hat on a letter, such as $\hat{B}$, this will denote an oriented group, that is, an element of $\widehat{\mathscr{C}}_{\mathbf{R}^{2}}(H)$.

(II.ii) Subgroups isomorphic to $\mathbf{R} \oplus \mathbf{Z}$. The space $\mathscr{C}_{\mathbf{R} \oplus \mathbf{Z}}(H)$ is the total space of a fibration

(6-6) $\mathscr{C}_{\mathbf{R} \oplus \mathbf{Z}}(H) \rightarrow \mathscr{C}_{\mathbf{R}^{2}}(H) \approx \mathbf{P}^{1}, A \mapsto \bar{A}, \quad$ with fibre $\left.\mathscr{C}_{\mathbf{R} \oplus \mathbf{Z}}(\mathbf{C}) \approx \mathbf{P}^{1} \times\right] 0, \infty[$.

To avoid confusion between the roles played by the two copies of $\mathbf{P}^{1}$ in (6-6), we will write $\mathbf{P}_{Z}^{1}$ for the one corresponding to $\mathscr{C}_{\mathbf{R}^{2}}(H)$ (maximal abelian subgroups of $H$ containing $Z(H)$ ) and write $\mathbf{P}_{\mathbf{R}}^{1}$ for the one corresponding to the choice of the $\mathbf{R}$-factor in $\mathscr{C}_{\mathbf{R} \oplus \mathbf{Z}}(\mathbf{C})$. Observe that the circle $\mathbf{S}^{1}$ that appears a few lines below double covers $\mathbf{P}_{Z}^{1}$.

Consider the two-fold cover $\widehat{\mathscr{C}}_{\mathbf{R} \oplus \mathbf{Z}}(H):=\{(A$, orientation on $\bar{A})\}$ of the total space of the previous fibration. The corresponding fibration $\widehat{\mathscr{C}}_{\mathbf{R} \oplus \mathbf{Z}}(H) \rightarrow \widehat{\mathscr{C}}_{\mathbf{R}^{2}}(H)$ 
is trivial. Indeed, an oriented plane $\hat{B} \subset H$ has a unique positive basis consisting of the unit central vector $(0,1)$ and a unit vector $\left(z_{0}, 0\right)$ orthogonal to $Z(H)$; the resulting canonical isomorphism from $\hat{B}$ onto $\mathbf{C}$ sends $(0,1) \in \hat{B}$ to $1 \in \mathbf{C}$ and $\left(z_{0}, 0\right) \in \hat{B}$ to $i \in \mathbf{C}$. Recall from Subsection 4.I that $\mathscr{C}_{\mathbf{R} \oplus \mathbf{Z}}(\mathbf{C})$ is homeomorphic to $\left.\mathbf{P}_{\mathbf{R}}^{1} \times\right] 0, \infty[$ (the open interval, ]0, 1[ in Subsection 4.I, is better rescaled as ]0, $\infty[$ here). It follows that

$$
\widehat{\mathscr{C}}_{\mathbf{R} \oplus \mathbf{Z}}(H)=\mathscr{C}_{\mathbf{R} \oplus \mathbf{Z}}(\mathbf{C}) \times \widehat{\mathscr{C}}_{\mathbf{R}^{2}}(H) \approx\left(\mathbf{P}_{\mathbf{R}}^{1} \times\right] 0, \infty[) \times \mathbf{S}^{1} .
$$

The total space $\mathscr{C}_{\mathbf{R} \oplus \mathbf{Z}}(H)$ of the fibration (6-6) is the quotient of $\widehat{\mathscr{C}}_{\mathbf{R} \oplus \mathbf{Z}}(H)$ by the orientation-reversing $\mathbf{Z} / 2 \mathbf{Z}$-action.

Consider a closed subgroup $A \cong \mathbf{R} \oplus \mathbf{Z}$ of $H$. Complex conjugation exchanges the maps $A \rightarrow \mathbf{C}$ corresponding to the two orientations of $\bar{A}$. Thus, if

$$
\left.(\theta, \rho, \varphi) \in \mathbf{P}_{\mathbf{R}}^{1} \times\right] 0, \infty\left[\times \mathbf{S}^{1} \approx \widehat{\mathscr{C}}_{\mathbf{R} \oplus \mathbf{Z}}(H)\right.
$$

are the coordinates corresponding to one orientation, the other orientation corresponds to coordinates $(\sigma(\theta), \rho, \varphi+\pi)$, where $\theta \mapsto \sigma(\theta)$ denotes the symmetry of $\mathscr{C}_{\mathbf{R} \oplus \mathbf{Z}}(\mathbf{C}) \approx \mathbf{P}_{\mathbf{R}}^{1}$ that replaces the chosen $\mathbf{R}$-factor with its complex conjugate, and the coordinate $\rho$ is the inverse of the appropriate minimal norm (as described in Subsection 4.I). Hence

$$
\begin{aligned}
\mathscr{C}_{\mathbf{R} \oplus \mathbf{Z}}(H) & \approx\left(\mathbf{P}_{\mathbf{R}}^{1} \times\right] 0, \infty\left[\times \mathbf{S}^{1}\right) /((\theta, \rho, \varphi) \sim(\sigma(\theta), \rho, \varphi+\pi)) \\
& \approx \mathbf{K} \times] 0, \infty[.
\end{aligned}
$$

Consequently, (6-6) is the direct product with ]0, $\infty$ [ of the standard fibration of the Klein bottle $\mathbf{K}$ over the projective line $\mathbf{P}_{Z}^{1}$, in other words, of the nontrivial circle bundle over the circle.

(II.iii) The closure of $\mathscr{C}_{\mathbf{R} \oplus \mathbf{Z}}(H)$. The frontier of $\mathscr{C}_{\mathbf{R} \oplus \mathbf{Z}}(H)$ in $\mathscr{C}(H)$ is the union of two pieces, namely $\mathscr{C}_{\mathbf{R}}(H)$ and $\mathscr{C}_{\mathbf{R}^{2}}(H)$. Convergence to points in the first piece occurs when the $\rho$-coordinate tends to 0 , while convergence to points in the second piece arises when $\rho \rightarrow \infty$. Moreover, all frontier points $A$ arise as limits of sequences $\left(A_{n}\right)$ with $\bar{A}_{n}$ constant — equal to $\widetilde{A}$, say — with only the $\rho$-coordinate varying.

Given a closed subgroup $A$ of $H$ isomorphic to $\mathbf{R}$, there are two cases to distinguish. If $A \neq Z(H)$, then we define $\widetilde{A} \cong \mathbf{R}^{2}$ to be the subgroup generated by $A$ and $Z(H)$. But if $A=Z(H)$ then every subgroup in $\mathscr{C}_{\mathbf{R}^{2}}(H) \approx \mathbf{P}_{Z}^{1}$ is an equally strong candidate for $\widetilde{A}$. Hence the factor $\mathbf{K}$ in $\mathscr{C}_{\mathbf{R} \oplus \mathbf{Z}}(H)$ can be obtained from $\mathscr{C}_{\mathbf{R}}(H) \approx \mathbf{P}^{2}$ by blowing-up the point $Z(H)$ and making the identification $A \mapsto \widetilde{A}$ elsewhere. 
Thus $\mathscr{C}_{\mathbf{R}}(H) \approx \mathbf{P}^{2}$ is attached to the end of $\left.\mathscr{C}_{\mathbf{R} \oplus \mathbf{Z}}(H) \approx \mathbf{K} \times\right] 0, \infty[$ near 0 by means of the map $\mathbf{K} \rightarrow \mathbf{P}^{2}$ that blows-down the circle that appears in (6-8) as the image of $\{Z(H)\} \times \mathbf{S}^{1} \subset \mathbf{P}_{\mathbf{R}}^{1} \times \mathbf{S}^{1}$.

The attachment of $\mathscr{C}_{\mathbf{R}^{2}}(H) \approx \mathbf{P}^{1}$ to the end of $\mathscr{C}_{\mathbf{R} \oplus \mathbf{Z}}(H)$ where $\rho \rightarrow \infty$ is straightforward: $\mathscr{C}_{\mathbf{R} \oplus \mathbf{Z}, \mathbf{R}^{2}}(H)$ is homeomorphic to the quotient of $\left.\left.\mathbf{K} \times\right] 0, \infty\right]$ by the relation that identifies $(k, \infty)$ to $\left(k^{\prime}, \infty\right)$ whenever $k, k^{\prime} \in \mathbf{K}$ are in the same fibre of the natural fibration $\mathbf{K} \rightarrow\left(\mathbf{S}^{1} /(\varphi \sim \varphi+\pi)\right)$.

Thus $\mathscr{C}_{\mathbf{R}, \mathbf{R} \oplus Z, \mathbf{R}^{2}}(H)$, the closure of $\mathscr{C}_{\mathbf{R} \oplus \mathbf{Z}}(H)$ in $\mathscr{C}(H)$, is homeomorphic to the space obtained from $\mathbf{K} \times[0, \infty]$ by blowing down a circle in $\mathbf{K} \times\{0\}$ and by collapsing $\mathbf{K} \times\{\infty\}$ to $\mathbf{P}^{1}$ in the manner described above.

(II.iv) Subgroups isomorphic to $\mathbf{Z}^{2}$. As in (II.ii), the space $\mathscr{C}_{\mathbf{Z}^{2}}(H)$ is the total space of a fibration

$$
\mathscr{C}_{\mathbf{Z}^{2}}(H) \rightarrow \mathscr{C}_{\mathbf{R}^{2}}(H) \approx \mathbf{P}^{1}, A \mapsto \bar{A} \quad \text { with fibre } \mathscr{L}(\mathbf{C}) \approx \mathbf{C}^{2} \backslash \Sigma,
$$

where $\Sigma$ denotes as in Section 4 the complex affine curve of equation $a^{3}-27 b^{2}=0$.

For $A \in \mathscr{C}_{\mathbf{Z}^{2}}(H)$, the two orientations of $\bar{A}$ correspond, as before, to conjugate embeddings $A \rightarrow \mathbf{C}$. The values taken by the classical invariants $g_{2}$ and $g_{3}$ on the image of these two embeddings are conjugate. Hence

$$
\widehat{\mathscr{C}}_{\mathbf{Z}^{2}}(H)=\mathscr{L}(\mathbf{C}) \times \widehat{\mathscr{C}}_{\mathbf{R}^{2}}(H) \approx\left(\mathbf{C}^{2} \backslash \Sigma\right) \times \mathbf{S}^{1}
$$

and

$$
\mathscr{C}_{\mathbf{Z}^{2}}(H) \approx\left(\left(\mathbf{C}^{2} \backslash \Sigma\right) \times \mathbf{S}^{1}\right) /\left(\left(g_{2}, g_{3}, \varphi\right) \sim\left(\bar{g}_{2}, \bar{g}_{3}, \varphi+\pi\right)\right) .
$$

(II.v) Subgroups of rank 2. We have a fibration

$$
\mathscr{C}_{\mathbf{Z}^{2}, \mathbf{R} \oplus \mathbf{Z}, \mathbf{R}^{2}}(H) \rightarrow \mathscr{C}_{\mathbf{R}^{2}}(H) \approx \mathbf{P}^{1}, A \mapsto \bar{A} \quad \text { with fibre } \mathscr{C}_{\mathbf{Z}^{2}, \mathbf{R} \oplus \mathbf{Z}, \mathbf{R}^{2}}(\mathbf{C}) .
$$

Set $\widehat{\mathscr{C}}_{\mathbf{Z}^{2}, \mathbf{R} \oplus \mathbf{Z}, \mathbf{R}^{2}}(H)=\widehat{\mathscr{C}}_{\mathbf{Z}^{2}}(H) \cup \widehat{\mathscr{C}}_{\mathbf{R} \oplus \mathbf{Z}}(H) \cup \widehat{\mathscr{C}}_{\mathbf{R}^{2}}(H)$. As in Subsections (II.ii) and (II.iv), the two-fold cover of (6-12)

$$
\widehat{\mathscr{C}}_{\mathbf{Z}^{2}, \mathbf{R} \oplus \mathbf{Z}, \mathbf{R}^{2}}(H) \rightarrow \widehat{\mathscr{C}}_{\mathbf{R}^{2}}(H)
$$

is a trivial fibration. Recall from Section 4 that $\mathscr{C}_{\mathbf{Z}}, \mathbf{R} \oplus \mathbf{Z}, \mathbf{R}^{2}(\mathbf{C})$ is homeomorphic to the complement of a 2-disc in a 4-sphere, that is,

$$
\mathscr{C}_{\mathbf{Z}^{2}, \mathbf{R} \oplus \mathbf{Z}, \mathbf{R}^{2}}(\mathbf{C}) \simeq\left(\mathbf{C}^{2} \cup\{\infty\}\right) \backslash \Sigma_{-},
$$

where $\Sigma_{-}=\left\{(a, b) \in \mathbf{C}^{2} \mid a^{3}=27 b^{2}\right.$ and $\left.|a|^{2}+|b|^{2} \leq 1\right\}$ is a 2 -disc $\mathbf{D}^{2}$ (as before, $\simeq$ denotes a homotopy equivalence). Thus,

$$
\begin{aligned}
\widehat{\mathscr{C}}_{\mathbf{Z}^{2}, \mathbf{R} \oplus \mathbf{Z}, \mathbf{R}^{2}}(H) & =\mathscr{C}_{\mathbf{Z}^{2}, \mathbf{R} \oplus \mathbf{Z}, \mathbf{R}^{2}}(\mathbf{C}) \times \widehat{\mathscr{C}}_{\mathbf{R}^{2}}(H) \\
& \approx\left(\left(\mathbf{C}^{2} \cup\{\infty\}\right) \backslash \Sigma_{-}\right) \times \mathbf{S}^{1} \approx\left(\mathbf{S}^{4} \backslash \mathbf{D}^{2}\right) \times \mathbf{S}^{1},
\end{aligned}
$$


where the embedding of $\mathbf{D}^{2}$ in $\mathbf{S}^{4}$ is not locally flat. Reflection in the real axis in $\mathbf{C}$ (which corresponds to changing the orientation of $\bar{A}$ ) acts on $\mathscr{C}(\mathbf{C}) \approx \mathbf{C}^{2} \cup\{\infty\}$ as $(a, b) \rightarrow(\bar{a}, \bar{b})$. Thus the space $\mathscr{C}_{\mathbf{Z}^{2}, \mathbf{R} \oplus \mathbf{Z}, \mathbf{R}^{2}}(H)$ is homeomorphic to

$$
\left(\left(\left(\mathbf{C}^{2} \cup\{\infty\}\right) \backslash \Sigma_{-}\right) \times \mathbf{S}^{1}\right) /((a, b, \varphi) \sim(\bar{a}, \bar{b}, \varphi+\pi)) .
$$

In particular, the space ${ }^{\mathscr{C}} \mathbf{Z}^{2}, \mathbf{R} \oplus \mathbf{Z}, \mathbf{R}^{2}(H)$ is a topological manifold of dimension 5.

(II.vi) Closure of subgroups of rank 2. The closure in $\mathscr{C}(H)$ of $\mathscr{C}_{\mathbf{Z}^{2}}(H)$ is the whole space $\mathscr{A}(H)$ of abelian closed subgroups. More precisely:

Proposition 6.1. (i) The space $A(H)$ of abelian closed subgroups of $H$ is homeomorphic to

$$
\mathscr{A}(H) \approx\left(\mathbf{S}^{4} \times \mathbf{S}^{1}\right) /\left((x, \varphi) \sim\left(x, \varphi^{\prime}\right) \text { if } x \in I\right),
$$

where $I \subset \mathbf{S}^{4}$ is a tame closed interval.

(ii) Every $\operatorname{Aut}(H)$-orbit in $\mathscr{C}_{\mathbf{Z}^{2}}(H)$ is dense in $\mathscr{A}(H)$.

Proof. (i) Let us introduce the two spaces

$$
\begin{aligned}
& \widehat{\mathscr{A}}_{\mathrm{en}}(H) \doteqdot\left\{(A, \hat{B}) \in \mathscr{A}(H) \times \widehat{\mathscr{C}}_{\mathbf{R}^{2}}(H) \mid A \subset \hat{B}\right\}, \\
& \mathscr{A}_{\mathrm{en}}(H) \doteqdot\left\{(A, B) \in \mathscr{A}(H) \times \mathscr{C}_{\mathbf{R}^{2}}(H) \mid A \subset B\right\},
\end{aligned}
$$

where "en" stands for "enhanced". There is an obvious two-sheeted covering $\widehat{\mathscr{A}}_{\mathrm{en}}(H) \rightarrow \mathscr{A}_{\mathrm{en}}(H)$ and an equally obvious projection $\mathscr{A}_{\mathrm{en}}(H) \rightarrow \mathscr{A}(H)$.

For each $\hat{B}_{0} \in \widehat{\mathscr{C}}_{\mathbf{R}^{2}}(H) \approx \mathbf{S}^{1}$, we have a canonical identification of $\hat{B}_{0}$ with $\mathbf{C}$, as in (II.ii), so the subspace of $\widehat{A}_{\mathrm{en}}(H)$ consisting of pairs $\left(A, \hat{B}_{0}\right)$ is canonically identified with $\mathscr{C}(\mathbf{C})$; moreover, the change of orientation in $\widehat{\mathscr{C}}_{\mathbf{R}^{2}}(H)$ corresponds to the involution $\sigma$ that complex conjugation induces on $\mathscr{C}(\mathbf{C})$. Thus

$$
\mathscr{A}_{\mathrm{en}}(H) \approx\left(\mathscr{C}(\mathbf{C}) \times \mathbf{S}^{1}\right) /((C, \varphi) \sim(\sigma(C), \varphi+\pi)) .
$$

And by employing the homeomorphism $f^{-1}$ of Theorem 4.6 we get

$$
\mathscr{A}_{\mathrm{en}}(H) \approx\left(\left(\mathbf{C}^{2} \cup\{\infty\}\right) \times \mathbf{S}^{1}\right) /(((a, b), \varphi) \sim((\bar{a}, \bar{b}), \varphi+\pi)) .
$$

For $\varphi \in \mathbf{S}^{1}$, let $\rho_{\varphi}$ be the homeomorphism of $\mathbf{C}^{2} \cup\{\infty\}$ defined by

$$
\rho_{\varphi}\left(a_{1}+i a_{2}, b_{1}+i b_{2}\right)=\left(a_{1}+i\left(a_{2} \cos \varphi-b_{2} \sin \varphi\right), b_{1}+i\left(a_{2} \sin \varphi+b_{2} \cos \varphi\right)\right) .
$$

Observe that the complex conjugate of $\rho_{\varphi}(a, b)$ is $\rho_{\varphi+\pi}(a, b)$. Define, then, a homeomorphism $R$ of $\left(\mathbf{C}^{2} \cup\{\infty\}\right) \times \mathbf{S}^{1}$ by

$$
R((a, b), \varphi)=\left(\rho_{\varphi}(a, b), \varphi\right) .
$$


Writing $s:((a, b), \varphi) \mapsto((\bar{a}, \bar{b}), \varphi+\pi)$ for the involution of $\left(\mathbf{C}^{2} \cup\{\infty\}\right) \times \mathbf{S}^{1}$ that appears in (6-15), we have

$R^{-1} s R((a, b), \varphi)=R^{-1} s\left(\rho_{\varphi}(a, b), \varphi\right)=R^{-1}\left(\rho_{\varphi+\pi}(a, b), \varphi+\pi\right)=((a, b), \varphi+\pi)$.

Thus we obtain a homeomorphism

$$
\begin{aligned}
\mathscr{A}_{\mathrm{en}}(H) & \approx\left(\left(\mathbf{C}^{2} \cup\{\infty\}\right) \times \mathbf{S}^{1}\right) /\{s\} \\
& \approx\left(\left(\mathbf{C}^{2} \cup\{\infty\}\right) \times \mathbf{S}^{1}\right) /\left\{R^{-1} s R\right\} \\
& =\left(\mathbf{C}^{2} \cup\{\infty\}\right) \times \mathbf{P}^{1} .
\end{aligned}
$$

The points in the right-hand term of (6-15) corresponding to pairs $(A, B) \in \mathscr{A}_{\mathrm{en}}(H)$ with $A \subset Z(H)$ are the points $((a, b), \varphi)$ with $a, b \in \mathbf{R}, a, b \geq 0, a^{2}+b^{2} \leq 1$, and $a^{3}=27 b^{2}$. (This follows from Section 4 , since $A$ is isomorphic to one of $\{0\}, \mathbf{Z}$, or $\mathbf{R}$, and the corresponding $C \in \mathscr{C}(\mathbf{C})$ is invariant under the action of $\sigma$.)

Near each $(A, B) \in \mathscr{A}_{\mathrm{en}}(H)$ with $A \not \subset Z(H)$, the projection $\mathscr{A}_{\mathrm{en}}(H) \rightarrow \mathscr{A}(H)$ is a local homeomorphism, and if $A \subset Z(H)$ then all pairs $(A, B) \in \mathscr{A}_{\mathrm{en}}(H)$ have the same image in $\mathscr{A}(H)$. It follows that

$\mathscr{A}(H) \approx\left(\left(\mathbf{C}^{2} \cup\{\infty\}\right) \times \mathbf{P}^{1}\right) /\left(((a, b), \psi) \sim\left((a, b), \psi^{\prime}\right)\right.$ for $\left.(a, b) \in I, \psi, \psi^{\prime} \in \mathbf{P}^{1}\right)$,

where $I$ is the tame arc

$$
I=\left\{(a, b) \in \mathbf{R}^{2} \mid a, b \geq 0, a^{2}+b^{2} \leq 1, a^{3}=27 b^{2}\right\}
$$

of $\left(\mathbf{C}^{2} \cup\{\infty\}\right) \times \mathbf{P}^{1}$. This ends the proof of (i).

(ii) For any closed subgroup $C_{0}$ of $H$, let $\overline{\operatorname{Aut}(H) C_{0}}$ be the closure in $\mathscr{C}(H)$ of its $\operatorname{Aut}(H)$-orbit. Let $\mathbf{R} \times \mathbf{R}$ denote the closed subgroup $\{(x+i y, t) \in H \mid y=0\}$ of $H$, and note that its $\operatorname{Aut}(H)$-orbit is the whole of $\mathscr{C}_{\mathbf{R}^{2}}(H)$. (Indeed the subgroup $\mathrm{SO}(2)$ has only one orbit in $\mathscr{C}_{\mathbf{R}^{2}}(H)$.) Thus, in order to prove (ii), it suffices to show that for any closed subgroup $C_{0}$ of $\mathbf{R} \times \mathbf{R}$ isomorphic to $\mathbf{Z}^{2}$, the subspace $\left\{C \in \overline{\operatorname{Aut}(H) C_{0}} \mid C \subset \mathbf{R} \times \mathbf{R}\right\}$ coincides with $\{A \in \mathscr{A}(H) \mid A \subset \mathbf{R} \times \mathbf{R}\}$. We will do this in three steps.

Step one. Let $P$ be the group of automorphisms of $\mathbf{R} \times \mathbf{R}$ that extend to automorphisms of $H$. We regard $P$ as a subgroup of the full automorphism group $\mathrm{GL}(\mathbf{R} \times \mathbf{R})$ of $\mathbf{R} \times \mathbf{R}$ (which should not be confused with the subgroup of $\operatorname{Aut}(H)$ previously denoted $\mathrm{GL}_{2}(\mathbf{R})$ ). As any automorphism of $H$ leaves the centre invariant, $P$ consists entirely of matrices of the form $\left(\begin{array}{c}* 0 \\ * *\end{array}\right)$. Now, from the diagonal matrices $\operatorname{diag}(a, b)$ in the subgroup $\mathrm{GL}_{2}(\mathbf{R})$ of $\operatorname{Aut}(H)$, we can obtain any diagonal matrix $\operatorname{diag}(a, a b)$ in $P$. On the other hand, using inner automorphisms $\operatorname{Int}_{(-i v, *)}:(x, t) \mapsto(x, t+v x)$, we can obtain any unipotent matrix $\left(\begin{array}{ll}1 & 0 \\ v & 1\end{array}\right)$ in $P$. It follows that $P$ is the full group of lower triangular matrices in $\operatorname{GL}(\mathbf{R} \times \mathbf{R})$. 
Step two. Let $G^{+}$be the subgroup of $\operatorname{GL}(\mathbf{R} \times \mathbf{R})$ consisting of matrices with positive determinant, and let $P^{+}=P \cap G^{+}$. As $G^{+}$can be seen as the set of oriented bases of $\mathbf{R} \times \mathbf{R}$, there is a projection $G^{+} \rightarrow \mathscr{L}(\mathbf{R} \times \mathbf{R})$ mapping a matrix to the lattice generated by its columns; this is a principal bundle whose structure group is $\mathrm{SL}_{2}(\mathbf{Z})$, acting on $G^{+}$by right multiplication. The group $G^{+}$itself acts on the right on the projective line $\mathbf{P}^{1}$ of $\mathbf{R} \times \mathbf{R}$ by

$$
\mathbf{P}^{1} \times G^{+} \rightarrow \mathbf{P}^{1}, \quad\left(\mathbf{R}(x, t),\left(\begin{array}{ll}
a & b \\
c & d
\end{array}\right)\right) \mapsto \mathbf{R}(x a+t c, x b+t d) .
$$

This action is transitive, and the isotropy group of the second factor $Z(H)$ of $\mathbf{R} \times \mathbf{R}$ is $P^{+}$. Thus we may identify $P^{+} \backslash G^{+}$with $\mathbf{P}^{1}$. The double coset space $P^{+} \backslash G^{+} / \mathrm{SL}_{2}(\mathbf{Z})$ can be viewed equally as the set of $P^{+}$-orbits in the quotient space $G^{+} / \mathrm{SL}_{2}(\mathbf{Z}) \approx \mathscr{L}(\mathbf{R} \times \mathbf{R})$ and as the set of $\mathrm{SL}_{2}(\mathbf{Z})$-orbits in the projective line $P^{+} \backslash G^{+} \approx \mathbf{P}^{1}$.

Step three. It is well known that the natural action of $\mathrm{SL}_{2}(\mathbf{Z})$ on the projective line $\mathbf{P}^{1}$ is minimal, that is, all its orbits are dense. Here is an $a d$ hoc argument: Let $t$ be an arbitrary point in $\mathbf{P}^{1}=\mathbf{R} \cup\{\infty\}$, and let $F=\overline{\mathrm{SL}_{2}(\mathbf{Z}) t}$ denote its orbit closure. Since $\left(\begin{array}{ll}1 & n \\ 0 & 1\end{array}\right) t \rightarrow \infty$ when $n \rightarrow \infty$, we have $\infty \in F$. For $a / b \in \mathbf{Q}$, with $a$ and $b$ in $\mathbf{Z}$ and coprime, there exist $c, d \in \mathbf{Z}$ with $a d-b c=1$, namely with $\left(\begin{array}{ll}a & c \\ b & d\end{array}\right) \in \mathrm{SL}_{2}(\mathbf{Z})$, and $a / b=\left(\begin{array}{ll}a & c \\ b & d\end{array}\right) \infty \in F$, so that $\mathbf{Q} \subset F$ and therefore $F=\mathbf{P}^{1}$.

It follows from step two that the action of $P^{+}$on $\mathscr{L}(\mathbf{R} \times \mathbf{R})$ is also minimal. In particular, the orbit closure $\overline{P^{+} C_{0}}$ contains the subspace $\mathscr{L}(\mathbf{R} \times \mathbf{R})$, and therefore coincides with $\{A \in \mathscr{A}(H) \mid A \subset \mathbf{R} \times \mathbf{R}\}$. A fortiori, we have

$$
\left\{C \in \overline{\operatorname{Aut}(H) C_{0}} \mid C \subset \mathbf{R} \times \mathbf{R}\right\}=\{A \in \mathscr{A}(H) \mid A \subset \mathbf{R} \times \mathbf{R}\} .
$$

\section{III. The space $\mathscr{C}_{\geq Z}(H)$ of subgroups that contain the centre.}

Each closed subgroup $C$ of $H$ that contains $Z(H)$ is uniquely determined by its image $p(C)$ in $\mathbf{C}$, and the assignment $C \mapsto p(C)$ gives a homeomorphism

$$
\mathscr{b}_{\geq Z}(H) \approx \mathscr{C}(\mathbf{C}) \approx \mathbf{S}^{4},
$$

where the homeomorphism of $\mathscr{C}(\mathbf{C})$ with a 4-sphere is the one from [Hubbard and Pourezza 1979]. More precisely, $\mathscr{C}_{\geq Z}(H) \approx \mathbf{S}^{4} \approx \Sigma \mathbf{S}^{3}$ is naturally the union of six subspaces:

(i) The central subgroup $Z(H)$ corresponds to the south pole.

(ii) The subgroups isomorphic to $\mathbf{R}^{2}$ correspond to a trefoil $T$ in $\mathbf{S}^{3}$.

(iii) The subgroups isomorphic to $\mathbf{R} \oplus \mathbf{Z}$ correspond to points on half-meridians between points of $T$ and the south pole.

(iv) The whole group $H$ corresponds to the north pole. 
(v) The subgroups $C \in \mathscr{C}(H)$ with $p(C) \cong \mathbf{R} \oplus \mathbf{Z}$ correspond to points on halfmeridians between points of $T$ and the north pole.

(vi) The subgroups in $\mathscr{L}_{\infty}(H)$, namely the inverse images in $H$ of lattices in $\mathbf{C}$, correspond to the complement of $\Sigma T$ in $\Sigma \mathbf{S}^{3}$, which is open and dense in $\mathscr{b}_{\geq Z}(H)$.

We introduce here more notation:

- the union of the subspaces in (i), (ii), and (iii) is a closed disc $\mathbf{D}_{-}$inside $\mathscr{b}_{\geq Z}(H)$,

- the union of the subspaces in (ii), (iv), and (v) is a closed disc $\mathbf{D}_{+}$inside $\mathscr{b}_{\geq Z}(H)$.

For reference in Section 8, we record part of the previous discussion as follows:

Lemma 6.2. With the above notation,

$$
\mathscr{C}_{\geq Z}(H) \cap \mathscr{A}(H)=\mathbf{D}_{-}=\left\{C \in \mathscr{C}_{\geq Z}(H) \mid p(C) \in \mathscr{C}_{\{0\}, \mathbf{Z}, \mathbf{R}}(\mathbf{C})\right\} .
$$

6.IV. $\operatorname{Aut}(H)$-orbits in $\mathscr{A}(H) \cup_{D_{-}} \mathscr{C}_{\geq Z}(H)$.

In the next proposition, the items are numbered in accordance with Theorem 1.4.

Proposition 6.3. For the natural action of the automorphism group $\operatorname{Aut}(H)$ on $\mathscr{C}(H)$, we have

(i) the one-point orbit $\{e\}$;

(ii) two $\operatorname{Aut}(H)$-orbits on $\mathscr{C}_{\mathbf{R}}(H) \approx \mathbf{P}^{2}$, see (6-1);

(iii) two $\operatorname{Aut}(H)$-orbits on $\left.\mathscr{C}_{\mathbf{Z}}(H) \approx \mathbf{P}^{2} \times\right] 0, \infty[$, see $(6-2)$;

(iv) one $\operatorname{Aut}(H)$-orbit on $\mathscr{C}_{\mathbf{R}^{2}}(H) \approx \mathbf{P}^{1}$, see (6-4);

(v) two $\operatorname{Aut}(H)$-orbits on $\left.\mathscr{C}_{\mathbf{R} \oplus \mathbf{Z}}(H) \approx \mathbf{K} \times\right] 0$, $\infty$ [, see (6-6) and (6-8);

(vi) uncountably many $\operatorname{Aut}(H)$-orbits on $\mathscr{C}_{\mathbf{Z}^{2}}(H)$, see (6-9) and (6-11);

(vii) one orbit in $p_{*}^{-1}\left(\mathscr{C}_{\mathbf{R} \oplus \mathbf{Z}}(\mathbf{C})\right)$;

(viii) the one-point orbit $H$;

(ix) and one orbit in $\mathscr{L}_{\infty}(H)$.

Moreover, for any point in $\mathscr{b}(H)$, its orbit under the identity component of $\operatorname{Aut}(H)$ coincides with its orbit under the whole of $\operatorname{Aut}(H)$.

Comments. The details of the proof are left to the reader but we wish to highlight the following points.

In $\mathscr{C}_{\mathbf{R}}(H)$, one orbit is a single point, the centre; the other orbit is its complement. In $\mathscr{C}_{\mathbf{Z}}(H)$, one orbit consists of central subgroups, the other of noncentral ones. Of the two orbits in $\mathscr{C}_{\mathbf{R} \oplus \mathbf{Z}}(H)$, one is 2-dimensional and consists of the subgroups whose identity component is $Z(H)$; the other orbit is 3-dimensional. 
Concerning the uncountably many orbits in $\mathscr{C}_{\mathbf{Z}^{2}}(H)$ : The space $\mathscr{C}_{\mathbf{Z}^{2}}(H)$ is 5-dimensional whereas the quotient of $\operatorname{Aut}(H)$ by the isotropy subgroup of a point in $\mathscr{C}_{\mathbf{Z}^{2}}(H)$ is only 4-dimensional.

The orbits of $\operatorname{Aut}(H)$ in $\mathscr{C}_{\geq Z}(H)$ are in natural bijection with the orbits of $\mathrm{GL}_{2}(\mathbf{R})$ in $\mathscr{C}(\mathbf{C})$. In particular, there are six orbits in $\mathscr{C}_{\geq Z}(H)$, namely

- the centre $Z(H)$, which is one of the two $\operatorname{Aut}(H)$-orbits in $\mathscr{C}_{\mathbf{R}}(H)$,

- the space $\mathscr{C}_{\mathbf{R}^{2}}(H)$,

- one of the two $\operatorname{Aut}(H)$-orbits in $\mathscr{C}_{\mathbf{R} \oplus \mathbf{Z}}(H)$,

- the whole group $H$,

- the space $p_{*}^{-1}\left(\mathscr{C}_{\mathbf{R} \oplus \mathbf{Z}}(\mathbf{C})\right)$, and

- the space $\mathscr{L}_{\infty}(H)$.

The dimension of these orbits is $0,1,2,0,2,4$, respectively.

The preceding proposition accounts for all of the Aut $(H)$-orbits in $\mathscr{C}(H)$ except for those in $\mathscr{L}_{n}(H)$, which will be described in Proposition 7.2.

\section{The structure of the space of lattices in the real Heisenberg group}

We remind the reader that the space of lattices $\mathscr{L}(H)$ is a disjoint union of the subspaces $\mathscr{L}_{n}(H)$ defined by declaring that a lattice $\Lambda$ is in $\mathscr{L}_{n}(H)$ if $[\Lambda, \Lambda]$ has index $n$ in $\Lambda \cap Z(H)$. In this section we shall describe the structure of the spaces $\mathscr{L}_{n}(H)$. The map

$$
p_{n}: \mathscr{L}_{n}(H) \rightarrow \mathscr{L}(\mathbf{C})
$$

from Section 5 will play a prominent role in our discussion, so we remind the reader that this is the projection induced by $p: H \rightarrow \mathbf{C}$.

Example 7.1. For an integer $n \geq 1$, the subgroup $\Lambda_{n}$ of $H$ generated by

$$
(1,0), \quad(i, 0), \quad \text { and } \quad(0,1 / n)
$$

is a lattice in $H$, and $\Lambda_{n} \in \mathscr{L}_{n}(H)$. Moreover, as a subset of $H=\mathbf{C} \times \mathbf{R}$,

$$
\Lambda_{n}=\mathbf{Z}[i] \times \frac{1}{n} \mathbf{Z} \quad \text { if } n \text { is even }
$$

and

$$
\Lambda_{n}=\left\{\left(x+i y, \frac{1}{2 n} t\right) \in \mathbf{Z}[i] \times \frac{1}{2 n} \mathbf{Z} \mid x y \equiv t(\bmod 2)\right\} \quad \text { if } n \text { is odd }
$$


In all cases ( $n$ even and odd), the lattice $\Lambda_{n}$ corresponds to

$$
\left(\begin{array}{ccc}
1 & \mathbf{Z} & \frac{1}{n} \mathbf{Z} \\
0 & 1 & \mathbf{Z} \\
0 & 0 & 1
\end{array}\right) \subset\left(\begin{array}{ccc}
1 & \mathbf{R} & \mathbf{R} \\
0 & 1 & \mathbf{R} \\
0 & 0 & 1
\end{array}\right)
$$

in the matrix picture.

Proof. Let us assume that $n$ is odd, and let us show the equality in (7-3). The verification of the other claims is left to the reader.

Denote by $\widetilde{\Lambda}_{n}$ the right-hand term of (7-3). By definition, and since both the commutator $[(1,0),(i, 0)]$ and $(0,1 / n)$ are central, $\Lambda_{n}$ is the set of elements of the form

$$
(1,0)^{x}(i, 0)^{y}(0,1 / n)^{s}=\left(x+i y, \frac{1}{2} x y+\frac{s}{n}\right) \quad \text { with } x, y, s \in \mathbf{Z} .
$$

Set $t \doteqdot 2 n\left(\frac{1}{2} x y+\frac{s}{n}\right)$ and observe that $t=n x y+2 s \equiv x y(\bmod 2)$, so that the element (7-4) is in $\widetilde{\Lambda}_{n}$. This shows that $\Lambda_{n} \subset \widetilde{\Lambda}_{n}$.

Conversely, if $\left(x+i y, \frac{1}{2 n} t\right) \in \widetilde{\Lambda}_{n}$, set $s \doteqdot \frac{1}{2}(t-n x y)$, and observe that $s \in \mathbf{Z}$, so that

$$
\left(x+i y, \frac{1}{2 n} t\right)=(1,0)^{x}(i, 0)^{y}(0,1 / n)^{s} \in \Lambda_{n} .
$$

This shows that $\widetilde{\Lambda}_{n} \subset \Lambda_{n}$.

Proposition 7.2. For every positive integer $n$, the space $\mathscr{L}_{n}(H)$ is a homogeneous space for the group $\operatorname{Aut}(H)$.

Proof. Let $\Lambda \in \mathscr{L}_{n}(H)$ and let $\Lambda_{n}$ be as in Example 7.1. We have to find $g \in \operatorname{Aut}(H)$ such that $g(\Lambda)=\Lambda_{n}$.

Since $\mathrm{GL}_{2}(\mathbf{R})$ acts transitively on the space of lattices in $\mathbf{C}$, there exists a $g \in \mathrm{GL}_{2}(\mathbf{R}) \subset \operatorname{Aut}(H)$ such that $p_{n}(g \Lambda)=p_{n}\left(\Lambda_{n}\right)=\mathbf{Z}[i] \subset \mathbf{C}$. Thus, without loss of generality, we can assume that $p_{n}(\Lambda)=\mathbf{Z}[i]$, namely, that $\Lambda$ has three generators of the form $(1, s),(i, t)$, and $(0,1 / n)$. The inner automorphism $\operatorname{Int}_{(-t+i s, 0)}$ maps these three generators to $(1,0),(i, 0)$, and $(0,1 / n)$, respectively, and therefore $\operatorname{Int}_{(-t+i s, 0)}(\Lambda)=\Lambda_{n}$.

A pleasant property of $\Lambda_{n}$ is that it corresponds to a familiar lattice in the matrix picture. But we shall see below that the lattice of the next example serves as a more convenient basepoint when one studies the isotropy of the $\operatorname{Aut}(H)$ action on $\Lambda_{n}$. We'll say more about this in Remark 7.6.

Example 7.3. For an integer $n \geq 1$, the subgroup $\Lambda_{n}^{\prime}$ of $H=\mathbf{C} \times \mathbf{R}$ generated by

$$
(1,1 / 2), \quad(i, 1 / 2), \quad(0,1 / n)
$$

is a lattice in $H$, and $\Lambda_{n}^{\prime} \in \mathscr{L}_{n}(H)$. Moreover

$$
\Lambda_{n}^{\prime}=\Lambda_{n}
$$


if $n$ is even, and

$$
\Lambda_{n}^{\prime}=\left\{\begin{array}{l|l}
\left(x+i y, \frac{1}{2 n} t\right) \in \mathbf{Z}[i] \times \frac{1}{2 n} \mathbf{Z} & \begin{array}{l}
t \text { even if } \frac{1}{2}(x+i y) \in \mathbf{Z}[i] \\
t \text { odd otherwise }
\end{array}
\end{array}\right\} \neq \Lambda_{n}
$$

if $n$ is odd.

Proof. The equality in (7-5) follows from the fact that, if $n$ is even, $(0,1 / 2)$ is a power of $(0,1 / n)$. We assume from now on that $n$ is odd.

Denote by $\widetilde{\Lambda}_{n}^{\prime}$ the set described explicitly in (7-6). By definition, $\Lambda_{n}^{\prime}$ is the set of elements of the form

$$
\left(1, \frac{1}{2}\right)^{x}\left(i, \frac{1}{2}\right)^{y}\left(0, \frac{1}{n}\right)^{s}=\left(x+i y, \frac{1}{2}(x+y+x y)+\frac{s}{n}\right) \quad \text { with } x, y, s \in \mathbf{Z} .
$$

Set $t \doteqdot 2 n\left(\frac{1}{2}(x+y+x y)+\frac{s}{n}\right)$ and observe that

$$
t=n(x+y+x y)+2 s \equiv(x+1)(y+1)-1 \quad(\bmod 2),
$$

so that the element (7-7) is in $\widetilde{\Lambda}_{n}^{\prime}$. This shows that $\Lambda_{n}^{\prime} \subset \widetilde{\Lambda}_{n}^{\prime}$. The opposite inclusion is proved similarly. (Compare with the proof of Example 7.1.)

To check that $\Lambda_{n}^{\prime} \neq \Lambda_{n}$, observe for example that $(1,1 /(2 n))$ and $(i, 1 /(2 n))$ are in $\Lambda_{n}^{\prime}$ and not in $\Lambda_{n}$, while $(1,0)$ and $(i, 0)$ lie in $\Lambda_{n}$ but not in $\Lambda_{n}^{\prime}$.

We will describe now the action of the automorphism group of $H$ on spaces of lattices.

Consider $w=(u, v) \in \mathbf{R}^{2}$, corresponding to an inner automorphism $\operatorname{Int}_{(w, *)}$ as in (5-4), and let $g=\left(\begin{array}{ll}a & b \\ c & d\end{array}\right) \in \mathrm{GL}_{2}(\mathbf{R})$ be viewed as an automorphism of $H$ as in (5-7). We will denote by

$$
\Phi_{w, g}=\left(\operatorname{Int}_{(w, *)}\right) g \in \operatorname{Aut}(H)
$$

the resulting composition. From Proposition 5.1, we have

$$
\Phi_{w, g} \Phi_{w^{\prime}, g^{\prime}}=\Phi_{w+g\left(w^{\prime}\right), g g^{\prime}}
$$

For an integer $n \geq 1$, we set

$$
S_{n}^{\prime}=\left\{\Phi_{w, g} \in \operatorname{Aut}(H) \mid w \in\left(\frac{1}{n} \mathbf{Z}\right)^{2} \text { and } g \in \mathrm{GL}_{2}(\mathbf{Z})\right\} .
$$

We leave it to the reader to check that $S_{n}^{\prime}$ is a subgroup of $\operatorname{Aut}(H)$ and that we have a natural split extension

$$
\{0\} \rightarrow\left(\frac{1}{n} \mathbf{Z}\right)^{2} \longrightarrow S_{n}^{\prime} \longrightarrow \mathrm{GL}_{2}(\mathbf{Z}) \rightarrow\{1\},
$$

where the homomorphisms are given by $w \mapsto \Phi_{w \text {,id }}$ and $\Phi_{w, g} \mapsto g$.

Proposition 7.4. For each integer $n \geq 1$, the subgroup $S_{n}^{\prime}$ of $\operatorname{Aut}(H)$ is the stabilizer of the lattice $\Lambda_{n}^{\prime} \in \mathscr{L}_{n}(H)$. 
Proof. Denote by $\operatorname{Stab}_{n}^{\prime}$ the stabilizer of $\Lambda_{n}^{\prime}$ in $\operatorname{Aut}(H)$; we have to show that $\mathrm{Stab}_{n}^{\prime}=S_{n}^{\prime}$.

Consider $\Phi_{w, g} \in \operatorname{Aut}(H)$, as in (7-8). Then $\Phi_{w, g}=\Phi_{w, \text { id }} \Phi_{0, g}$. The actions of $\Phi_{0, g}$ and $\Phi_{w, \text { id }}$ on the first two generators of $\Lambda_{n}^{\prime}$ are given by

$$
\Phi_{0, g}\left(1, \frac{1}{2}\right)=\left(a+i c, \frac{1}{2}\right), \quad \Phi_{0, g}\left(i, \frac{1}{2}\right)=\left(b+i d, \frac{1}{2}\right),
$$

and, for $w=(u, v)$,

$$
\begin{aligned}
\Phi_{(u, v), \text { id }}\left(1, \frac{1}{2}\right)-\left(1, \frac{1}{2}\right) & =-n v\left(0, \frac{1}{n}\right), \\
\Phi_{(u, v), \text { id }}\left(i, \frac{1}{2}\right)-\left(i, \frac{1}{2}\right) & =n u\left(0, \frac{1}{n}\right) .
\end{aligned}
$$

Suppose first that $\Phi_{w, g} \in S_{n}^{\prime}$. Since $(a, c) \notin(2 \mathbf{Z})^{2}$ and $(b, d) \notin(2 \mathbf{Z})^{2}$, the equalities of (7-11) and similar equalities for $\Phi_{0, g^{-1}}$ imply that $\Phi_{0, g} \in \operatorname{Stab}_{n}^{\prime}$. Since $n(-v, u) \in \mathbf{Z}^{2}$, the equalities of (7-12) imply that $\Phi_{w, \text { id }} \in \operatorname{Stab}_{n}^{\prime}$. Hence $\Phi_{w, g} \in \mathrm{Stab}_{n}^{\prime}$.

Suppose now that $\Phi_{w, g} \in \operatorname{Stab}_{n}^{\prime}$. Then $g$ preserves $p_{n}\left(\Lambda_{n}^{\prime}\right)=\mathbf{Z}[i]$, so that $g \in$ $\mathrm{GL}_{2}(\mathbf{Z})$ and $\Phi_{0, g} \in S_{n}^{\prime}$. Using the previous step, we have $\Phi_{0, g} \in S_{n}^{\prime} \subset \mathrm{Stab}_{n}^{\prime}$, hence $\Phi_{w, \text { id }}=\Phi_{w, g}\left(\Phi_{0, g}\right)^{-1} \in \operatorname{Stab}_{n}^{\prime}$. The equalities of (7-12) imply that $w \in\left(\frac{1}{n} \mathbf{Z}\right)^{2}$. It follows that $\Phi_{g, w} \in S_{n}^{\prime}$.

Remark 7.5. Proposition 7.4 implies that the extensions (7-10) corresponding to different values of $n$ are semidirect products; indeed they are all isomorphic to the obvious extension

$$
\{0\} \rightarrow \mathbf{Z}^{2} \longrightarrow \mathbf{Z}^{2} \rtimes \mathrm{GL}_{2}(\mathbf{Z}) \longrightarrow \mathrm{GL}_{2}(\mathbf{Z}) \rightarrow\{1\}
$$

where the action of $\mathrm{GL}_{2}(\mathbf{Z})$ on $\mathbf{Z}^{2}$ is the standard one.

Proof. For each real number $\rho>0$, the map

$$
\mathbf{R}^{2} \rtimes \mathrm{GL}_{2}(\mathbf{R}) \rightarrow \mathbf{R}^{2} \rtimes \mathrm{GL}_{2}(\mathbf{R}), \quad(w, g) \mapsto(\rho w, g)
$$

is a group automorphism. It follows that, for various values of $n$, the split extensions

$$
\{0\} \rightarrow\left(\frac{1}{n} \mathbf{Z}\right)^{2} \longrightarrow S_{n}^{\prime} \longrightarrow \mathrm{GL}_{2}(\mathbf{Z}) \rightarrow\{1\}
$$

are pairwise isomorphic.

Remark 7.6. For all $n \geq 1$, the following sequence splits:

$$
\{0\} \rightarrow\left(\frac{1}{n} \mathbf{Z}\right)^{2} \longrightarrow \operatorname{Stab}\left(\Lambda_{n}\right) \longrightarrow \mathrm{GL}_{2}(\mathbf{Z}) \rightarrow\{1\} .
$$

However, the splitting is more cumbersome in this case, which is why we prefer to work with $\Lambda_{n}^{\prime}$.

Proposition 7.7. (i) For every integer $n \geq 1$, the projection $p_{n}: \mathscr{L}_{n}(H) \rightarrow \mathscr{L}(\mathbf{C})$ of (7-1) is a fibre bundle with fibre a 2-torus. 
(ii) For two integers $n, n^{\prime}$, the two fibre bundles $p_{n}, p_{n^{\prime}}$ are isomorphic over the identity $\mathscr{L}(\mathbf{C}) \rightarrow \mathscr{L}(\mathbf{C})$.

In particular, for any pair $n, n^{\prime}$ of positive integers, the total spaces $\mathscr{L}_{n}(H)$ and $\mathscr{L}_{n^{\prime}}(H)$ are homeomorphic to each other.

Proof. (i) Given $L_{0} \in \mathscr{L}(\mathbf{C})$, we choose a positively oriented $\mathbf{Z}$-basis $\left(z_{0}, z_{0}^{\prime}\right)$ for it. Each lattice $L$ in a small neighbourhood $U \subset \mathscr{L}(\mathbf{C})$ of $L_{0}$ has a unique positively oriented $\mathbf{Z}$-basis $\left(z, z^{\prime}\right)$ close to $\left(z_{0}, z_{0}^{\prime}\right)$, and the commutator in $H$ of elements of the form $(z, *)$ and $\left(z^{\prime}, *\right)$ is $\left(0, \operatorname{Im}\left(\bar{z} z^{\prime}\right)\right)=(0$, area $(\mathbf{C} / L))$, which is close to $\left(0, \operatorname{area}\left(\mathbf{C} / L_{0}\right)\right)$.

For any choice of $r, r^{\prime} \in\left[0, \frac{1}{n}\left[\right.\right.$, let $\Lambda_{r, r^{\prime}} \in \mathscr{L}_{n}(H)$ be the lattice generated by $\left(z, r \operatorname{Im}\left(\bar{z} z^{\prime}\right)\right), \quad\left(z^{\prime}, r^{\prime} \operatorname{Im}\left(\bar{z} z^{\prime}\right)\right)$, and $\left(0, \frac{1}{n} \operatorname{Im}\left(\bar{z} z^{\prime}\right)\right)$. Then $p\left(\Lambda_{r, r^{\prime}}\right)=L$ and $\Lambda_{r, r^{\prime}}=\Lambda_{s, s^{\prime}}$ if and only if $n\left(r-s, r^{\prime}-s^{\prime}\right) \in \mathbf{Z} \times \mathbf{Z}$. Thus $\left(z, z^{\prime}, r, r^{\prime}\right)$ provide coordinates for a trivialisation near $L_{0}$.

(ii) Consider the automorphism $\Psi_{n}$ of $\operatorname{Aut}(H)$ defined by

$$
\Psi_{n}\left(\Phi_{w, g}\right)=\Phi_{w / n, g}
$$

In Proposition 7.4 we proved that the stabilizer of $\Lambda_{n}^{\prime}$ in $\operatorname{Aut}(H)$ is $S_{n}^{\prime}$, and it is clear from the definition of $S_{n}^{\prime}$ that

$$
\Psi\left(S_{1}^{\prime}\right)=S_{n}^{\prime}
$$

If $\Pi: \operatorname{Aut}(H) \rightarrow \mathrm{GL}_{2}(\mathbf{R})$ is as in (5-3), observe that

$$
\Pi \Psi_{n}=\Pi
$$

Denote by $\pi_{n}^{\prime}$ the restriction of $\Pi$ to $S_{n}^{\prime}$. The diagram

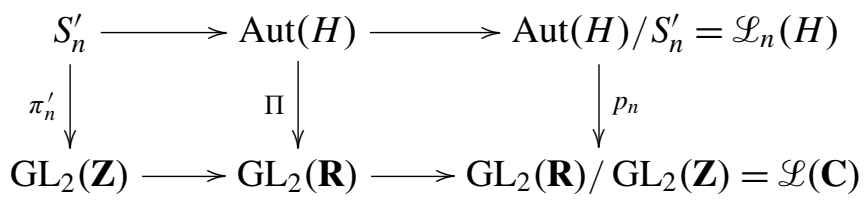

is commutative. By (7-14), if we apply the homomorphism $\Psi_{n}^{-1}$ to $S_{n}^{\prime}$ and $\operatorname{Aut}(H)$, and the identity to the groups and space of the bottom row of (7-16), we find the diagram corresponding to (7-16) for the value $n=1$. It follows that the bundles $p_{n}$ and $p_{1}$ are isomorphic over the identity of $\mathscr{L}(\mathbf{C})$.

Let us now point out a natural factorisation of the spaces $\mathscr{L}_{n}(H)$. The group of positive real numbers acts naturally on $\mathscr{L}(\mathbf{C})$, see Section 4 , as well as on $H$ by $(s,(z, t)) \mapsto\left(s z, s^{2} t\right)$, see Section 5 . The latter action induces free actions on 
$\mathscr{L}(H)$ and $\mathscr{L}_{\infty}(H)$, and we have homeomorphisms

$$
\begin{aligned}
\mathscr{L}_{n}(H) & \approx \mathscr{L}_{n}^{\text {umod }}(H) \times \mathbf{R}_{+}^{*} \text { for all } n \geq 1, \\
\mathscr{L}_{\infty}(H) & \approx \mathscr{L}_{\infty}^{\text {umod }}(H) \times \mathbf{R}_{+}^{*},
\end{aligned}
$$

where the superscript "umod" indicates that the lattice $p_{n}(\Lambda)$ in $\mathbf{C}$ is unimodular for $\Lambda \in \mathscr{L}_{n}^{\text {umod }}(H)$, and similarly for $\Lambda \in \mathscr{L}_{\infty}^{\text {umod }}(H)$. The mapping $p_{n}$ introduced above, as well as the homeomorphism $p_{\infty}: \mathscr{L}_{\infty}(H) \rightarrow \mathscr{L}(\mathbf{C})$, have restrictions, again denoted by the same letters

$$
\begin{aligned}
& p_{n}: \mathscr{L}_{n}^{\text {umod }}(H) \rightarrow \mathscr{L}^{\text {umod }}(\mathbf{C}) \quad \text { for all } n \geq 1, \\
& p_{\infty}: \mathscr{L}_{\infty}^{\text {umod }}(H) \stackrel{\approx}{\rightarrow} \mathscr{L}^{\text {umod }}(\mathbf{C}) .
\end{aligned}
$$

These mappings are $\mathrm{SL}_{2}(\mathbf{R})$-equivariant; since $\mathrm{SL}_{2}(\mathbf{R})$ acts transitively on the space $\mathscr{L}^{\text {umod }}(\mathbf{C})$, it follows that these mappings are also onto.

Let $\operatorname{SAut}(H)$ denote the subgroup of $\operatorname{Aut}(H)$ of those automorphisms $\Phi$ which induce on $H / Z(H)$ an orientation-preserving and area-preserving automorphism. Then $\operatorname{SAut}(H)$ is isomorphic to the standard semidirect product $\mathbf{R}^{2} \rtimes \mathrm{SL}_{2}(\mathbf{R})$. For each integer $n \geq 1$, the proofs of Propositions 7.2 and 7.4 show that $\mathscr{L}_{n}^{\text {umod }}(H)$ is a homogeneous space $\operatorname{SAut}(H) / J_{n}$ and that we have a split exact sequence

$$
\{0\} \rightarrow\left(\frac{1}{n} \mathbf{Z}\right)^{2} \longrightarrow J_{n}=\left(\frac{1}{n} \mathbf{Z}\right)^{2} \rtimes \mathrm{SL}_{2}(\mathbf{Z}) \longrightarrow \mathrm{SL}_{2}(\mathbf{Z}) \rightarrow\{1\},
$$

where $J_{n}=S_{n}^{\prime} \cap \operatorname{SAut}(H)$. The group $J_{1}=\mathbf{Z}^{2} \rtimes \mathrm{SL}_{2}(\mathbf{Z})$ appears in analytical number theory as the Jacobi group; see [Eichler and Zagier 1985, Section 1.1].

As in Section 4, we denote by $\widetilde{\mathrm{SL}}_{2}(\mathbf{R})$ the universal covering of $\mathrm{SL}_{2}(\mathbf{R})$, by $\widetilde{\mathrm{SL}}_{2}(\mathbf{Z})$ the inverse image of $\mathrm{SL}_{2}(\mathbf{Z})$ in this group, and by $\mathbf{R}^{2} \rtimes \widetilde{\mathrm{SL}}_{2}(\mathbf{R})$ the standard semidirect product, which can be identified with the universal covering group of $\operatorname{SAut}(H)$. The space $\mathscr{L}_{n}^{\text {umod }}(H)$ is also diffeomorphic to the homogeneous space

$$
\left(\mathbf{R}^{2} \rtimes{\widetilde{\mathrm{SL}_{2}}}_{2}(\mathbf{R})\right) /\left(\mathbf{Z}^{2} \rtimes{\widetilde{\mathrm{SL}_{2}}}_{2}(\mathbf{Z})\right) .
$$

As $\mathbf{R}^{2} \rtimes \widetilde{\mathrm{SL}}_{2}(\mathbf{R})$ is a contractible space, it follows that $\mathscr{L}_{n}^{\text {umod }}(H)$ is an EilenbergMac Lane space with fundamental group isomorphic to $\mathbf{Z}^{2} \rtimes \widetilde{\mathrm{SL}_{2}}(\mathbf{Z})$. In view of the factorisations (7-17) and of the last claim of Proposition 7.7, we have proved the following proposition.

Proposition 7.8. For any positive integer $n$, the space $\mathscr{L}_{n}(H)$ is a 6-dimensional connected manifold that is an Eilenberg-Mac Lane space with fundamental group $\mathbf{Z}^{2} \rtimes{\widetilde{\mathrm{SL}_{2}}}_{2}(\mathbf{Z})$.

The proof Theorem 1.3 (i)-(iii) is now complete: See Proposition 7.8 for (i), about $\mathscr{L}(H)$, Proposition 6.1 for (ii), about $\mathscr{A}(H)$, and Subsection 6.III for (iii), about $\mathscr{C}_{\geq Z}(H)$. 


\section{On the global structure of the space $\mathscr{C}(H)$}

In this section we shall describe the closure of the space of lattices in $H$ and complete the proof of the theorems stated in the introduction. We begin with an analysis of the closure of the strata $\mathscr{L}_{n}(H)$. For each $n$, the frontier $\partial \mathscr{L}_{n}(H)$ is as described in Theorem 1.4; it contains all of the nonlattice subgroups of $H$ except those in $\mathscr{L}_{\infty}(H)$. In Proposition 8.5, we show that $\mathscr{L}_{\infty}(H)$ lies in the closure of the union of the $\mathscr{L}_{n}(H)$, thus completing the proof that the lattices form an open dense subset of $\mathscr{C}(H)$. This also leads easily to a proof that $\mathscr{C}(H)$ is not locally connected at points of $\mathscr{L}_{\infty}(H)$.

Recall from the proof of Proposition 6.1 that we denote by $\mathbf{R} \times \mathbf{R}$ the closed subgroup $\{(x+i y, t) \in H \mid y=0\}$ of $H$.

8.I. The frontier of $\mathscr{L}_{n}(H)$, the frontier of $\bigcup_{n \geq 1} \mathscr{L}_{n}(H)$, and related matters. Let $n$ be a fixed positive integer.

We consider a sequence $\left(A_{k}\right)_{k \geq 1}$ of closed subgroups of $\mathbf{R} \times \mathbf{R}$, a closed subgroup $A$ of $\mathbf{R} \times \mathbf{R}$, and a sequence $\left(t_{k}\right)_{k \geq 1}$ of nonzero real numbers with the following properties:

- $A_{k} \cong \mathbf{Z}^{2}$ for all $k \geq 1$ and $A \cong \mathbf{Z}^{2}$;

- $A_{k} \cap Z(H)=\left\langle\left(0, t_{k}\right)\right\rangle$ for all $k \geq 1$;

- $A_{k} \rightarrow A$ and $\left|t_{k}\right| \rightarrow \infty$ for $k \rightarrow \infty$.

(Recall that $\langle\cdots\rangle$ indicates the subgroup generated by $\cdots$.) An example of such data is provided by $A_{k}=\langle(1,0),(-1 / k, 1)\rangle, A=\mathbf{Z}^{2}$, and $t_{k}=k$.

Lemma 8.1. Let $\left(A_{k}\right)_{k \geq 1}, A$, and $\left(t_{k}\right)_{k \geq 1}$ be as above.

(i) There exists a sequence $\left(\Lambda_{k}\right)_{k \geq 1}$ in $\mathscr{L}_{n}(H)$ such that $\Lambda_{k} \cap(\mathbf{R} \times \mathbf{R})=A_{k}$ for all $k \geq 1$.

(ii) For any $\left(\Lambda_{k}\right)_{k \geq 1}$ as in (i), we have $\lim _{k \rightarrow \infty} \Lambda_{k}=A$ in $\mathscr{C}(H)$.

Proof. (i) Let $k \geq 1$. Since $A_{k} \cap Z(H) \cong \mathbf{Z}$, the projection $p\left(A_{k}\right) \subset \mathbf{R} \subset \mathbf{C}$ is a closed subgroup isomorphic to $\mathbf{Z}$. Choose $u_{k} \in \mathbf{R}^{*}$ such that $p\left(A_{k}\right)=\left\langle u_{k}\right\rangle$; observe that $A_{k}$ is generated by $\left(u_{k}, s_{k}\right)$ and $\left(0, t_{k}\right)$ for some $s_{k} \in \mathbf{R}$. Define $\Lambda_{k}$ to be the subgroup of $H$ generated by $A_{k}$ and $\left(i n t_{k} / u_{k}, 0\right)$. Then $\Lambda_{k} \in \mathscr{L}_{n}(H)$ and $\Lambda_{k} \cap(\mathbf{R} \times \mathbf{R})=A_{k}$ for all $k \geq 1$.

(ii) Let $\left(\Lambda_{k}\right)_{k \geq 1}$ be as in (i). Three observations are in order.

First, the group $p(A)$ is not reduced to $\{0\}$, because $A \cong \mathbf{Z}^{2}$ is closed in $H$ (note that $p(A)$ need not be closed in $\mathbf{C}$ ); it follows that $\sup _{k \geq 1}\left|u_{k}\right|<\infty$. Second, coarea $\left(p\left(\Lambda_{k}\right)\right)=n\left|t_{k}\right|$ is arbitrarily large when $k \rightarrow \infty$. Finally,

$$
\operatorname{Im}(z) \in \mathbf{Z} \frac{\operatorname{coarea}\left(p\left(\Lambda_{k}\right)\right)}{\left|u_{k}\right|} \quad \text { for all } z \in p\left(\Lambda_{k}\right),
$$


so that

$$
|\operatorname{Im}(z)| \geq \frac{\operatorname{coarea}\left(p\left(\Lambda_{k}\right)\right)}{\left|u_{k}\right|} \quad \text { for all } z \in p\left(\Lambda_{k}\right), z \notin \mathbf{R} .
$$

It follows from the first two observations that the right-hand term of the last inequality tends to $\infty$ when $k$ tends to $\infty$.

Hence $\lim _{k \rightarrow \infty} \Lambda_{k}=\lim _{k \rightarrow \infty} \Lambda_{k} \cap p^{-1}(\mathbf{R})=\lim _{k \rightarrow \infty} A_{k}=A$.

Recall from Section 1 that $\mathscr{L}_{! !}(H)=\left(\bigcup_{n=1}^{\infty} \mathscr{L}_{n}(H)\right) \cup \mathscr{L}_{\infty}(H)$, from Lemma 6.2 that we have defined closed discs $\mathbf{D}_{-}, \mathbf{D}_{+} \subset \mathscr{C}_{\geq Z}(H)$, and that the interior of $\mathbf{D}_{+}$ is

$$
\stackrel{\circ}{\mathbf{D}}_{+}=\left\{C \in \mathscr{C}_{\geq Z}(H) \mid p(C) \in \mathscr{C}_{\mathbf{R} \oplus \mathbf{Z}, \mathbf{C}}(\mathbf{C})\right\}=\mathscr{C}_{\geq Z}(H) \backslash\left(\mathscr{L}_{\infty}(H) \cup \mathbf{D}_{-}\right) .
$$

Lemma 8.2. Let $\left(C_{k}\right)_{k \geq 1}$ be a sequence in $\mathscr{L}_{! !}(H)$ and $C \in \stackrel{\circ}{\mathbf{D}}_{+}$.

Then $\lim _{k \rightarrow \infty} C_{k}=C$ in $\mathscr{b}(H)$ if and only if $\lim _{k \rightarrow \infty} p\left(C_{k}\right)=p(C)$ in $\mathscr{C}(\mathbf{C})$. Proof. If $\lim _{k \rightarrow \infty} C_{k}=C$, then $\lim _{k \rightarrow \infty} p\left(C_{k}\right)=p(C)$ by the continuity of $p_{*}$; see Proposition 5.2.

If $\lim _{k \rightarrow \infty} p\left(C_{k}\right)=p(C)$, then $\lim _{k \rightarrow \infty} \operatorname{coarea}\left(p\left(C_{k}\right)\right)=\operatorname{coarea}(p(C))=0$; hence $\lim _{k \rightarrow \infty}\left(C_{k} \cap Z(H)\right)=Z(H)$, and it follows that $\lim _{k \rightarrow \infty} C_{k}=C$.

Proposition 8.3. (i) For each $n \geq 1$, the frontier of $\mathscr{L}_{n}(H)$ in $\mathscr{C}(H)$ is

$$
\mathscr{A}(H) \bigcup_{\mathbf{D}_{-}}\left(\mathscr{C}_{\geq Z}(H) \backslash \mathscr{L}_{\infty}(H)\right) .
$$

(ii) The closure of $\mathscr{L}_{\infty}(H)$ in $\mathscr{C}(H)$ is $\mathscr{C}_{\geq Z}(H)$.

Proof. We leave the proof of (ii) to the reader, and we split that of (i) in three steps.

First step: The frontier of $\mathscr{L}_{n}(H)$ contains $\mathscr{A}(H)$. Lemma 8.1 shows that this frontier contains at least one point of $\mathscr{C}_{\mathbf{Z}^{2}}(H)$. Since $\mathscr{L}_{n}(H)$ is invariant under the action of $\operatorname{Aut}(H)$, its frontier is also invariant, and contains therefore at least one $\operatorname{Aut}(H)$-orbit in $\mathscr{C}_{\mathbf{Z}^{2}}(H)$. It follows from Proposition 6.1(ii) that the frontier of $\mathscr{L}_{n}(H)$ contains $\mathscr{A}(H)$.

Second step: The frontier of $\mathscr{L}_{n}(H)$ contains $\stackrel{\circ}{\mathbf{D}}_{+}$. This is clear from Lemma 8.2, since $\mathscr{L}(\mathbf{C})$ is dense in $\mathscr{C}(\mathbf{C})$.

Third step. It remains to show that $\mathscr{L}_{\infty}(H)$ is disjoint from $\overline{\mathscr{L}_{n}(H)}$. This will be a consequence of Lemma 8.4(iii) below.

For an open subset $\mathscr{W}$ in $\mathscr{L}(\mathbf{C})$ and a positive integer $N$, set

$$
\boldsymbol{U}_{\mathscr{W}, N}=\left\{D \in\left(\bigcup_{n=N+1}^{\infty} \mathscr{L}_{n}(H)\right) \cup \mathscr{L}_{\infty}(H) \mid p(D) \in \mathscr{W}\right\} .
$$

Lemma 8.4. (i) For $\mathscr{W}$ and $N$ as above, the subset $\mathscr{U}_{\mathscr{W}, N}$ is open in $\mathscr{C}(H)$.

(ii) For $C \in \mathscr{L}_{\infty}(H)$, the set $\left\{\mathcal{U}_{\mathscr{W}, N}\right\}$, with $\mathscr{W} \ni p(C)$ and $N \geq 1$, is a basis of neighbourhoods of $C$ in $\mathscr{C}(H)$. 
(iii) Let $\left(C_{k}\right)_{k \geq 1}$ be a sequence in $\mathscr{L}(H)$ and $C \in \mathscr{L}_{\infty}(H)$; for $k \geq 1$, let $n_{k}$ be the integer such that $C_{k} \in \mathscr{L}_{n_{k}}(H)$.

Then $\lim _{k \rightarrow \infty} C_{k}=C$ if and only if $\lim _{k \rightarrow \infty} p\left(C_{k}\right)=p(C)$ and $\lim _{k \rightarrow \infty} n_{k}=\infty$.

Proof. (i) Recall from Proposition 5.3 and its proof that the functions

$$
J: \mathscr{L}_{! !}(H) \rightarrow \mathbf{R}_{+}, \quad J(C)= \begin{cases}1 / n & \text { if } D \in \mathscr{L}_{n}(H), \\ 0 & \text { if } D \in \mathscr{L}_{\infty}(H)\end{cases}
$$

and

$$
p_{*}: \mathscr{L}_{! !}(H) \rightarrow \mathscr{L}(\mathbf{C}), \quad D \mapsto p(D)
$$

are continuous. Hence the subset

$$
\boldsymbol{U}_{W, N}=J^{-1}\left(\left[0,1 / N[) \cap p_{*}^{-1}(\mathcal{W})\right.\right.
$$

is open in $\mathscr{C}(H)$.

(ii) Consider the function $\ell: H \rightarrow \mathbf{R}_{+}$defined by $\ell(z, t)=|z|+|t|$ and the function $\delta: H \times H \rightarrow \mathbf{R}_{+}$defined by

$$
\delta\left((z, t),\left(z^{\prime}, t^{\prime}\right)\right)=\ell\left((z, t)^{-1}\left(z^{\prime}, t^{\prime}\right)\right)=\left|z^{\prime}-z\right|+\left|t^{\prime}-t-\frac{1}{2} \operatorname{Im}\left(\bar{z} z^{\prime}\right)\right| .
$$

The function $\delta$ is not a distance function, because it does not satisfy the triangle inequality. But we go on as if it were (compare with (3-3)); thus, we set

$$
B_{R}=\{(z, t) \in H \mid \ell(z, t)<R\} \text { for all } R>0
$$

and we define for all $C \in \mathscr{C}(H)$ and $R, \epsilon>0$

$$
\mathscr{V}_{R, \epsilon}(C)=\left\{\begin{array}{l|l}
D \in \mathscr{C}(H) & \begin{array}{c}
\delta((z, t), D)<\epsilon \text { for all }(z, t) \in C \cap \bar{B}_{R} \\
\delta\left(\left(z^{\prime}, t^{\prime}\right), C\right)<\epsilon \text { for all }\left(z^{\prime}, t^{\prime}\right) \in D \cap \bar{B}_{R}
\end{array}
\end{array}\right\} .
$$

We leave it to the reader to check that $\left(\mathscr{V}_{R, \epsilon}(C)\right)_{R>0, \epsilon>0}$ is a basis of neighbourhoods of $C$ in $\mathscr{C}(H)$, each of which are relatively compact.

Consider $C \in \mathscr{L}_{\infty}(H)$, a pair $R, \epsilon>0$, and the resulting open neighbourhood $\mathscr{V}_{R, \epsilon}(C)$ of $C$. Set $\eta=\epsilon /(R+2)$ and

$$
\begin{aligned}
\mathscr{W} & =\left\{L \in \mathscr{V}_{R, \eta}(p(C)) \cap \mathscr{L}(\mathbf{C}) \mid \operatorname{coarea}(L)<2 \operatorname{coarea}(p(C))\right\}, \\
N & =\lceil 2 \operatorname{coarea}(p(C)) / \eta\rceil
\end{aligned}
$$

(where $\lceil\cdots\rceil$ indicates the upper integral part). To complete the proof, it suffices to show that $\mathcal{U}_{\mathscr{W}, N} \subset \mathscr{V}_{R, \epsilon}(C)$. In other words, we choose $D \in \mathcal{U}_{\mathscr{W}, N}$ and we have to verify the two conditions on $C$ and $D$ that appear in the right side of (8-1).

For $z, z^{\prime} \in \mathbf{C}$ with $|z|<R$ and $\left|z^{\prime}-z\right|<\eta$, note that

$$
\left|\operatorname{Im}\left(\bar{z} z^{\prime}\right)\right|=\left|\operatorname{Im}\left(\bar{z}\left(z^{\prime}-z\right)\right)\right|<R \eta .
$$


For $(z, t),\left(z^{\prime}, t^{\prime}\right) \in B_{R} \subset H$ with $\left|z^{\prime}-z\right|<\eta$ and $\left|t^{\prime}-t\right|<\eta$, note that

$$
\delta\left((z, t),\left(z^{\prime}, t^{\prime}\right)\right) \leq\left|z^{\prime}-z\right|+\left|t^{\prime}-t\right|+\frac{1}{2}\left|\operatorname{Im}\left(\bar{z} z^{\prime}\right)\right|<2 \eta+\frac{1}{2} R \eta<\epsilon .
$$

Consider now $\left(z^{\prime}, t^{\prime}\right) \in D$ with $\ell\left(z^{\prime}, t^{\prime}\right) \leq R$. Since $p(D) \in \mathscr{V}_{R, \eta}(p(C))$ and since $z^{\prime} \in p(D)$ satisfies $\left|z^{\prime}\right| \leq R$, there exists $z \in p(C)$ with $\left|z-z^{\prime}\right|<\eta$. We have $\left(z, t^{\prime}\right) \in C$ and $\delta\left(\left(z^{\prime}, t^{\prime}\right),\left(z, t^{\prime}\right)\right)<\epsilon$ by $(8-2)$. This shows that $\delta\left(\left(z^{\prime}, t^{\prime}\right), C\right)<\epsilon$.

Consider finally $(z, t) \in C$ with $\ell(z, t) \leq R$. Since $p(D) \in \mathscr{V}_{R, \eta}(p(C))$ and since $z \in p(C)$ satisfies $|z| \leq R$, there exists $z^{\prime} \in p(D)$ with $\left|z^{\prime}-z\right|<\eta$. Since $D \in \mathcal{U}_{\mathscr{W}, N}$, there exists $u \in \mathbf{R}$ such that $(0, u) \in D \cap Z(H)$ and

$$
|u| \leq \operatorname{coarea}(p(D)) / N \leq 2 \operatorname{coarea}(p(C)) / N,
$$

namely with $|u|<\eta$ (by the choice of $N$ ). Hence there exists $t^{\prime} \in \mathbf{R}$ with $\left(z^{\prime}, t^{\prime}\right) \in D$ and $\left|t^{\prime}-t\right|<\eta$. We have

$$
\delta\left((z, t),\left(z^{\prime}, t^{\prime}\right)\right)=\ell\left(z^{\prime}-z, t^{\prime}-t-\frac{1}{2} \operatorname{Im}\left(\bar{z} z^{\prime}\right)\right)<\left|z^{\prime}-z\right|+\left|t^{\prime}-t\right|+\frac{1}{2} R \eta<\epsilon .
$$

This shows that $\delta((z, t), D)<\epsilon$, and thus completes the proof of (ii).

(iii) This claim is a straightforward consequence of (ii).

Proposition 8.5. (i) The frontier of $\mathscr{L}(H)=\bigcup_{n=1}^{\infty} \mathscr{L}_{n}(H)$ contains $\mathscr{L}_{\infty}(H)$.

(ii) The space $\mathscr{C}(H)$ is not locally connected. Indeed, any point $C \in \mathscr{L}_{\infty}(H)$ does not have any connected neighbourhood contained inside $\mathscr{L}_{! !}(H)$.

(iii) The subspaces $\mathscr{L}(H)$ and $\mathscr{L}_{! !}(H)$ are open and dense in $\mathscr{C}(H)$.

(iv) The space $\mathscr{C}(H)$ is arc connected.

Proof. Claim (i) follows from Lemma 8.4(ii).

(ii) Let $C \in \mathscr{L}_{\infty}(H)$ and let $U$ be a neighbourhood of $C$ contained in $\mathscr{L}_{! !}(H)$. By Lemma 8.4, $U$ contains a neighbourhood $\mathcal{U}_{\mathscr{W}, N}$. The function $J$, from the proof of Proposition 5.3, is continuous on $U$, takes its values in $\{1, \ldots, 1 / n, \ldots, 0\}$, and is not constant on $U$ since it is not constant on $U_{\mathscr{W}, N}$. Hence $U$ is not connected, and this establishes (ii).

(iii) The spaces $\mathscr{L}(H)$ and $\mathscr{L}_{! !}(H)$ are open; see respectively Remark 3.5(ii) and Proposition 5.2(v). The closure of $\mathscr{L}(H)$ is the whole of $\mathscr{C}(H)$ by Proposition 8.3 and by (i).

(iv) First note that the space $\mathscr{D}(H)$ of discrete subgroups of $H$ is arc connected and contains $\{e\}$. Indeed, for positive $s \in \mathbf{R}$, denote by $\varphi_{s}$ the automorphism $(z, t) \mapsto\left(s z, s^{2} t\right)$ of $H$. Then we have $\lim _{s \rightarrow \infty} \varphi_{s}(\Gamma)=\{e\}$ in $\mathscr{D}(H)$ for each discrete subgroup $\Gamma$ of $H$. If $\Lambda$ is a lattice then we have also $\lim _{s \rightarrow 0} \varphi_{s}(\Lambda)=H$, so that $H$ lies in the path component of $\{e\}$. 
The path component of $\{e\}$ also contains $\mathscr{A}(H)$, see Proposition 6.1, while the path component of $H$ contains $\mathscr{C}_{\geq Z}(H)$ since the latter is homeomorphic to $\mathbf{S}^{4}$. Since $\mathscr{C}(H)$ is the union of $\mathscr{D}(H), \mathscr{A}(H)$, and $\mathscr{C}_{\geq Z}(H)$, this completes the proof of (iv).

\section{II. The space $\mathscr{L}_{! !}(\mathrm{H})$ as a bundle over $\mathscr{L}(\mathrm{C})$, and the homotopy type of $\mathscr{C}(H)$.} Let us denote by $\mathbf{L}$ the subset $\{1,1 / 2, \ldots, 1 / n, \ldots, 0\}$ of the real line, endowed with the induced topology from $\mathbf{R}$, which makes it a countable compact space. For any topological space $X$, denote by $\mathbf{L} X$ the quotient of $X \times \mathbf{L}$ by the equivalence relation $(x, 0) \sim\left(x^{\prime}, 0\right)$ for all $x, x^{\prime} \in X$ (we will resist writing more than once that $\mathbf{L} X$ is the "discrete cone" over $X$ ). Observe that if $X$ is compact, so is $\mathbf{L} X$. For a fibre bundle $p: E \rightarrow B$, with fibre $F$, let $\mathbf{L}_{\mathrm{bu}}(p): \mathbf{L}_{\mathrm{bu}} E \rightarrow B$ be the fibre bundle, with fibre $\mathbf{L} F$, of which the total space is the quotient of $E \times \mathbf{L}$ by the equivalence relation $(e, 0) \sim\left(e^{\prime}, 0\right)$ for $e, e^{\prime} \in E$ with $p(e)=p\left(e^{\prime}\right)$; we will denote by $p(e)$ the class of $(e, 0)$.

Proposition 8.6. The projection $p: \mathscr{L}_{! !}(H) \rightarrow \mathscr{L}(\mathbf{C})$ is a fibre bundle that is isomorphic to $\mathbf{L}_{\mathrm{bu}}\left(p_{1}\right): \mathbf{L}_{\mathrm{bu}} \mathscr{L}_{1}(H) \rightarrow \mathscr{L}(\mathbf{C})$.

Proof. For $n \geq 1$, denote by $\Theta_{n}: \mathscr{L}_{n}(H) \rightarrow \mathscr{L}_{1}(H)$ the bundle isomorphism of Proposition 7.7. Define $\Theta: \mathscr{L}_{! !}(H) \rightarrow \mathbf{L}_{\mathrm{bu}} \mathscr{L}_{1}(H)$ by

$$
\Theta(C)= \begin{cases}\left(\Theta_{n}(C), 1 / n\right) & \text { if } C \in \mathscr{L}_{n}(H), \\ p(C) & \text { if } C \in \mathscr{L}_{\infty}(H) .\end{cases}
$$

Then $\Theta$ is clearly a bijection above the identity on $\mathscr{L}(\mathbf{C})$. As its domain and its range are fibre bundles with compact fibres (which are of the form $\mathbf{L} T$, with $T$ a 2-torus) over a locally compact basis (the space $\mathscr{L}(\mathbf{C})$ ), it only remains to check that $\Theta$ is continuous at each point.

The continuity of $\Theta$ at a point $C \in \mathscr{L}_{n}(H)$ follows from the continuity of $\Theta_{n}$ and from the fact that $\mathscr{L}_{n}(H)$ is open in $\mathscr{L}_{! !}(H)$. The continuity of $\Theta$ at a point $C \in \mathscr{L}_{\infty}(H)$ follows from Lemma 8.4(ii).

Let $X$ be a topological space, $B$ an open dense subspace, and $p: E \rightarrow B$ a fibre bundle. We define the fibre-collapse continuation of $p$ over $X$ as the space $E_{*}=E \sqcup(X \backslash B)$ with the projection $p_{*}: E_{*} \rightarrow B$ defined by $p_{*}(e)=p(e)$ for $e \in E$ and $p_{*}(x)=x$ for $x \in X \backslash B$; the topology on $E_{*}$ is defined by decreeing that $E$ is open and that $x \in X \backslash B$ has a basis of open neighbourhoods consisting of the sets $p_{*}^{-1}(U)$, with $U$ an open neighbourhood of $x$ in $X$.

Proposition 8.7. The bundle projection $p: \mathscr{L}_{! !}(H) \rightarrow \mathscr{L}(C)$ extends to the continuous mapping

$$
p_{*}: \mathscr{C}(H) \backslash \mathscr{A}(H)=\mathscr{L}_{! !}(H) \cup \stackrel{\circ}{\mathbf{D}}_{+} \rightarrow \mathscr{L}(\mathbf{C}) \cup \mathscr{C}_{\mathbf{R} \oplus \mathbf{Z}, \mathbf{C}}(\mathbf{C})
$$


of Proposition 5.2(iii), which is isomorphic to the fibre-collapse continuation of $p$ over $\mathscr{L}(\mathbf{C}) \cup \mathscr{C}_{\mathbf{R} \oplus \mathbf{Z}, \mathbf{C}}(\mathbf{C})$. In particular, the restriction of $p_{*}$ to $\stackrel{\bigcirc}{\mathbf{D}}_{+}$is a homeomorphism onto $\mathscr{C}_{\mathbf{R} \oplus \mathbf{Z}, \mathbf{C}}(\mathbf{C})$.

One might hope that $p_{*}$ has an extension to $\mathscr{C}(H)$ showing that $\mathscr{C}_{\geq Z}(H)$ is a retract of $\mathscr{C}(H)$. The next proposition shows that this optimism is too naive. But all is not lost, as Proposition 8.9 shows that the desired retraction does exist at the level of homotopy.

Proposition 8.8. The mapping $p_{*}$ of Proposition 8.7 does not have a continuous extension $\mathscr{C}(H) \rightarrow \mathscr{C}(\mathbf{C})$.

Proof. If such an extension $\bar{p}_{*}: \mathscr{C}(H) \rightarrow \mathscr{C}(\mathbf{C})$ were to exist, it would be unique, since $A(H)$ is nowhere dense. Moreover, $\bar{p}_{*}$ would be equivariant with respect to the homomorphism $\Pi: \operatorname{Aut}(H) \rightarrow \mathrm{GL}_{2}(\mathbf{R})$ of (5-3), since this is the case for $p_{*}$.

Let $A_{k}=\langle(1,0),(-1 / k, 1)\rangle$ for $k \geq 1$, and let $A=\mathbf{Z}^{2}$ be as just before Lemma 8.1. Let $\left(\Lambda_{k}\right)_{k \geq 1}$ be the corresponding sequence of lattices, and note Lemma 8.1(ii). Then $\lim _{k \rightarrow \infty} p\left(\Lambda_{k}\right)=\mathbf{R} \subset \mathbf{C}$, and therefore $\bar{p}_{*}\left(\mathbf{Z}^{2}\right)=\mathbf{R}$.

Let $\left(\varphi_{s}\right)_{s>0}$ be the automorphisms of $H$ from the proof of Proposition 8.5. Then $\lim _{s \rightarrow \infty} \varphi_{s}\left(\mathbf{Z}^{2}\right)=\{e\}$ and $\varphi_{s}(\mathbf{R})=\mathbf{R}$ for all $s>0$. Hence $\bar{p}_{*}(\{e\})=\mathbf{R}$. But this is impossible, since the action of $\operatorname{Aut}(H)$ on $\{e\}$ is trivial and the action of $\mathrm{GL}_{2}(\mathbf{R})$ on $\mathbf{R}$ is not.

We denote by

$$
q_{*}: \mathscr{C}(H) \backslash \mathscr{A}(H)=\mathscr{L}_{! !}(H) \cup \stackrel{\circ}{\mathbf{D}}_{+} \rightarrow \mathscr{L}_{\infty}(H) \cup \stackrel{\circ}{\mathbf{D}}_{+}
$$

the composition of $p_{*}$ with the natural homeomorphism from $\mathscr{L}(\mathbf{C}) \cup \mathscr{C}_{\mathbf{R} \oplus \mathbf{Z}, \mathbf{C}}(\mathbf{C})$ onto $\mathscr{L}_{\infty}(H) \cup \stackrel{\circ}{\mathbf{D}}_{+}$.

Let $\mathscr{C}(H) / \mathscr{A}(H)$ denote the compact space obtained from $\mathscr{C}(H)$ by identifying all points of $\mathscr{A}(H)$ with each other. Let similarly $\mathscr{C}_{\geq Z}(H) / \mathbf{D}_{-}$denote the compact space obtained from $\mathscr{C}_{\geq Z}(H)$ by identifying all points of $\mathbf{D}_{-}$with each other; observe that it is the union of $\mathscr{L}_{\infty}(H) \cup \dot{\mathbf{D}}_{+}=\mathscr{C}_{\geq Z}(H) \backslash \mathbf{D}_{-}$and the point [D_] (the class of $\mathbf{D}_{-}$).

We denote by

$$
q_{*}^{\text {ext }}: \mathscr{C}(H) / \mathscr{A}(H) \rightarrow \mathscr{C}_{\geq Z}(H) / \mathbf{D}_{-}
$$

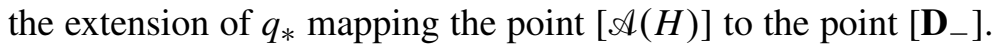

Proposition 8.9. (i) With the notation above, $q_{*}^{\text {ext }}$ is a retraction.

(ii) There exists a continuous map $\psi: \mathscr{C}_{\geq Z}(H) / \mathbf{D}_{-} \rightarrow \mathscr{b}_{\geq Z}(H)$ such that the composition

$$
\mathscr{C}_{\geq Z}(H) \rightarrow \mathscr{C}_{\geq Z}(H) / \mathbf{D}_{-} \stackrel{\psi}{\longrightarrow} \mathscr{C}_{\geq Z}(H)
$$

is homotopic to the identity. 
(iii) The composition

$$
\mathscr{C}(H) \rightarrow \mathscr{C}(H) / \mathscr{A}(H) \stackrel{q_{*}^{\mathrm{ext}}}{\longrightarrow} \mathscr{C}_{\geq Z}(H) / \mathbf{D}_{-} \stackrel{\psi}{\longrightarrow} \mathscr{C}_{\geq Z}(H)
$$

is a weak retraction.

(iv) The fourth homotopy group $\pi_{4}(\mathscr{C}(H))$ has an infinite cyclic quotient.

Proof. (i) For $C \in \mathscr{C}(H) \backslash \mathscr{A}(H)$, we have $q_{*}^{\text {ext }}(C)=\langle C, Z(H)\rangle$; this implies that the restriction of $q_{*}^{\text {ext }}$ to $\mathscr{C}_{\geq Z}(H) / \mathbf{D}_{-}$is the identity.

Note that $q_{*}^{\text {ext }}(C)=p^{-1}(p(C))$ for any $C \in \mathscr{C}(H) \backslash \mathscr{A}(H)$. We have to check that $q_{*}^{\text {ext }}$ is continuous at each point. The continuity of $q_{*}^{\text {ext }}$ at points distinct from $[\mathscr{A}(H)]$ results from the continuity of $C \mapsto p(C)$ at these points (Proposition 5.2) and the continuity of $p^{-1}$ as a mapping from closed subsets of $\mathbf{C}$ to closed subsets of $H$. It remains to check that $q_{*}^{\text {ext }}$ is continuous at $[\mathscr{A}(H)]$.

Let $\left(C_{k}\right)_{k \geq 1}$ be a sequence in $\mathscr{C}(H) \backslash \mathscr{A}(H)$ such that

$$
\lim _{k \rightarrow \infty} C_{k}=[\mathscr{A}(H)] \in \mathscr{C}(H) / \mathscr{A}(H) .
$$

Since the projection $p_{*}: \mathscr{C}(H) \backslash \mathscr{A}(H) \rightarrow \mathscr{C}(\mathbf{C}) \backslash \mathscr{C}_{\{0\}, \mathbf{Z}, \mathbf{R}}(\mathbf{C})$ is proper (see Proposition 8.7), it follows that $\lim _{k \rightarrow \infty} q_{*}^{\text {ext }}\left(C_{k}\right)=\left[\mathbf{D}_{-}\right]$.

(ii) In Subsection 6.III we saw that there is a homeomorphism from $\mathscr{C}_{\geq Z}(H)$ to the 4-sphere that identifies $\mathbf{D}_{-}$with a subset of a closed hemisphere $\mathbf{S}_{-}$. Thus the identity map of $\mathscr{b}_{\geq Z}(H)$ induces a continuous map $\mathscr{C}_{\geq Z}(H) / \mathbf{D}_{-} \rightarrow \mathscr{C}_{\geq Z}(H) / \mathbf{S}_{-}$, and we can define $\psi$ to be the composition of this map and a homotopy inverse to $\mathscr{b}_{\geq Z}(H) \rightarrow \mathscr{C}_{\geq Z}(H) / \mathbf{S}_{-}$.

(iii) The mapping of (8-4) is continuous as a composition of continuous maps, and its restriction to $\mathscr{b}_{\geq Z}(H)$ is homotopic to the identity by (i) and (ii).

(iv) This is a straightforward consequence of (iii) and of the homeomorphisms of $\mathscr{C}_{\geq Z}(H)$ with $\mathbf{S}^{4}$.

\section{Acknowledgments}

Bridson thanks his colleagues at the University of Geneva and the EPFL (Lausanne) for their hospitality throughout this project.

\section{References}

[Benedetti and Petronio 1992] R. Benedetti and C. Petronio, Lectures on hyperbolic geometry, Springer, Berlin, 1992. MR 94e:57015 Zbl 0768.51018

[Borel and Ji 2006] A. Borel and L. Ji, Compactifications of symmetric and locally symmetric spaces, Birkhäuser, Boston, 2006. MR 2007d:22030 Zbl 1100.22001 
[Bourbaki 1963] N. Bourbaki, Éléments de mathématique, Fascicule XXIX, Livre VI: Intégration, Chapitre 7: Mesure de Haar, Chapitre 8: Convolution et représentations, Actualités Scientifiques et Industrielles 1306, Hermann, Paris, 1963. MR 31 \#3539 Zbl 0156.03204

[Canary et al. 1987] R. D. Canary, D. B. A. Epstein, and P. Green, "Notes on notes of Thurston", pp. 3-92 in Analytical and geometric aspects of hyperbolic space (Coventry/Durham, 1984), edited by D. B. A. Epstein, London Math. Soc. Lecture Note Ser. 111, Cambridge Univ. Press, 1987. MR 89e:57008 Zbl 0612.57009

[Chabauty 1950] C. Chabauty, "Limite d'ensembles et géométrie des nombres", Bull. Soc. Math. France 78 (1950), 143-151. MR 12,479f Zbl 0039.04101

[Champetier 2000] C. Champetier, "L'espace des groupes de type fini”, Topology 39:4 (2000), 657680. MR 2001i:20084 Zbl 0959.20041

[Champetier and Guirardel 2005] C. Champetier and V. Guirardel, "Limit groups as limits of free groups", Israel J. Math. 146 (2005), 1-75. MR 2006d:20045 Zbl 1103.20026

[de Cornulier et al. 2007] Y. de Cornulier, L. Guyot, and W. Pitsch, "On the isolated points in the space of groups”, J. Algebra 307:1 (2007), 254-277. MR 2008c:20082 Zbl 1132.20018

[de Cornulier et al. 2008] Y. de Cornulier, L. Guyot, and W. Pitsch, "The space of subgroups of an abelian group", preprint, 2008. arXiv 0811.1549v1

[Eichler and Zagier 1985] M. Eichler and D. Zagier, The theory of Jacobi forms, Progress in Mathematics 55, Birkhäuser, Boston, 1985. MR 86j:11043 Zbl 0554.10018

[Fell 1962] J. M. G. Fell, "A Hausdorff topology for the closed subsets of a locally compact nonHausdorff space”, Proc. Amer. Math. Soc. 13 (1962), 472-476. MR 25 \#2573 Zbl 0106.15801

[Ghys 2007] É. Ghys, "Knots and dynamics", pp. 247-277 in International Congress of Mathematicians, I, edited by M. Sanz-Solé et al., Eur. Math. Soc., Zürich, 2007. MR 2008k:37001 Zbl 1125.37032

[Grigorchuk 1984] R. I. Grigorchuk, "Degrees of growth of finitely generated groups and the theory of invariant means", Izv. Akad. Nauk SSSR Ser. Mat. 48:5 (1984), 939-985. In Russian; translated in Math. USSR Izv. 25 (1985), 259-300. MR 86h:20041 Zbl 0583.20023

[Gromov 1981] M. Gromov, "Groups of polynomial growth and expanding maps", Inst. Hautes Études Sci. Publ. Math. 53 (1981), 53-73. MR 83b:53041 Zbl 0474.20018

[Guivarc'h and Rémy 2006] Y. Guivarc'h and B. Rémy, "Group-theoretic compactification of Bruhat-Tits buildings", Ann. Sci. École Norm. Sup. (4) 39:6 (2006), 871-920. MR 2008f:20056 Zbl 1126.20029

[Guivarc'h et al. 1998] Y. Guivarc'h, L. Ji, and J. C. Taylor, Compactifications of symmetric spaces, Progress in Mathematics 156, Birkhäuser, Boston, 1998. MR 2000c:31006 Zbl 1053.31006

[de la Harpe 2000] P. de la Harpe, Topics in geometric group theory, University of Chicago Press, 2000. MR 2001i:20081 Zbl 0965.20025

[Harvey 1977] W. J. Harvey, "Spaces of discrete groups", pp. 295-348 in Discrete groups and automorphic functions (Cambridge, 1975), edited by W. J. Harvey, Academic, London, 1977. MR 58 \#16961

[Hubbard and Pourezza 1979] J. Hubbard and I. Pourezza, "The space of closed subgroups of $\mathbb{R}^{2}$ ", Topology 18:2 (1979), 143-146. MR 80h:14023 Zbl 0429.57002

[Hurwitz 1964] A. Hurwitz, Vorlesungen über allgemeine Funktionentheorie und elliptische Funktionen, Grundlehren der Mathematischen Wissenschaften 3, Springer, Berlin, 1964. MR 30 \#3959 Zbl 0135.12101 
[Kaplansky 1971] I. Kaplansky, Lie algebras and locally compact groups, University of Chicago Press, 1971. MR 43 \#2145 Zbl 0223.17001

[Macbeath and Świerczkowski 1960] A. M. Macbeath and S. Świerczkowski, "Limits of lattices in a compactly generated group", Canad. J. Math. 12 (1960), 427-437. MR 22 \#6863 Zbl 0097.03301

[Milnor 1971] J. Milnor, Introduction to algebraic K-theory, Annals of Mathematics Studies 72, Princeton University Press, 1971. MR 50 \#2304 Zbl 0237.18005

[Mumford 1976] D. Mumford, Algebraic geometry, I: Complex projective varieties, Grundlehren der Mathematischen Wissenschaften 221, Springer, Berlin, 1976. MR 56 \#11992 Zbl 0356.14002

[Munkres 1975] J. R. Munkres, Topology: A first course, Prentice-Hall, Englewood Cliffs, N.J., 1975. MR 57 \#4063 Zbl 0306.54001

[Oler 1973] N. Oler, "Spaces of closed subgroups of a connected Lie group", Glasgow Math. J. 14 (1973), 77-79. MR 48 \#2305 Zbl 0266.22009

[Raghunathan 1972] M. S. Raghunathan, Discrete subgroups of Lie groups, Ergebnisse der Mathematik und ihrer Grenzgebiete 68, Springer, New York, 1972. MR 58 \#22394a Zbl 0254.22005

[Saks and Zygmund 1965] S. Saks and A. Zygmund, Analytic functions, 2nd ed., Monografie Matematyczne 28, Państwowe Wydawnietwo Naukowe, Warsaw, 1965. MR 31 \#4889 Zbl 0136.37301

[Thurston 1979] W. P. Thurston, “The geometry and topology of three-manifolds", lecture notes, Princeton University, 1979, Available at http://msri.org/publications/books/gt3m.

Received December 18, 2007.

MARTIN R. BRIDSON

MATHEMATICAL InSTITUTE

24-29 ST GILES'

OXFORD OX1 3LB

UNITED KINGDOM

bridson@maths.ox.ac.uk

PierRe DE LA HARPE

Section de Mathématiques, Université de Genève

C.P. 64

1211 GENÈVE 4

SWITZERLAND

Pierre.delaHarpe@unige.ch

VICTOR KLEPTSYN

UMR 6625 DU CNRS, IRMAR

CAMPUS DE BEAULIEU

35042 RENNES CEDEX

FRANCE

Victor.Kleptsyn@univ-rennes1.fr 